\title{
AFIP-7 Tomography - 2013 Status Report
}

\author{
A. E. Craft \\ W. J. Williams \\ M. I. K. Abir \\ D. M. Wachs
}

October 2013

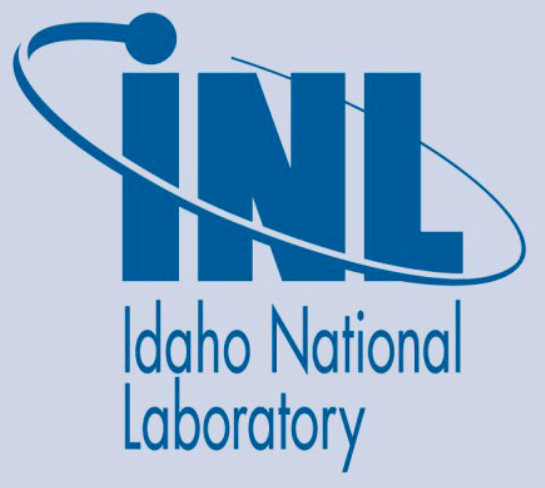

The INL is a U.S. Department of Energy National Laboratory operated by Battelle Energy Alliance 


\section{DISCLAIMER}

This information was prepared as an account of work sponsored by an agency of the U.S. Government. Neither the U.S. Government nor any agency thereof, nor any of their employees, makes any warranty, expressed or implied, or assumes any legal liability or responsibility for the accuracy, completeness, or usefulness, of any information, apparatus, product, or process disclosed, or represents that its use would not infringe privately owned rights. References herein to any specific commercial product, process, or service by trade name, trade mark, manufacturer, or otherwise, does not necessarily constitute or imply its endorsement, recommendation, or favoring by the U.S. Government or any agency thereof. The views and opinions of authors expressed herein do not necessarily state or reflect those of the U.S. Government or any agency thereof. 
INL/EXT-13-30741

Rev. 1

\title{
AFIP-7 Tomography - 2013 Status Report
}

\author{
A. E. Craft \\ W. J. Williams \\ M. I. K. Abir \\ D. M. Wachs
}

October 2013

\author{
Idaho National Laboratory \\ Idaho Falls, Idaho 83415 \\ http://www.inl.gov
}

\author{
Prepared for the \\ U.S. Department of Energy \\ Office of National Nuclear Security Administration \\ Under DOE Idaho Operations Office
}





\section{CONTENTS}

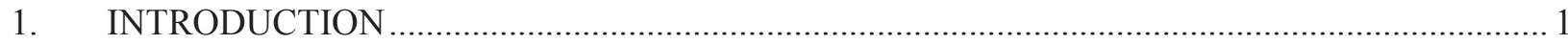

2. CURRENT NEUTRON IMAGING CAPABILITIES AT THE NRAD ....................................... 2

3. DEVELOPMENT OF MULTI-CHANNEL PLATE IMAGING SYSTEMS …........................... 5

3.1 Fundamentals of Multi-Channel Plate Neutron Imaging Systems ........................................ 5

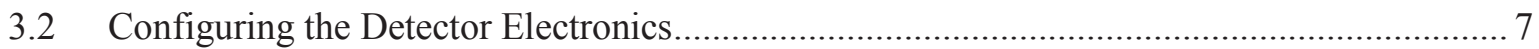

3.3 Gamma Sensitivity of the Multi-Channel Plate Detector..................................................... 11

3.4 Determining the Radiography Facility Category According to ASTM Standards................ 13

3.5 Tomographic Reconstruction of a Beam Purity Indicator ................................................ 17

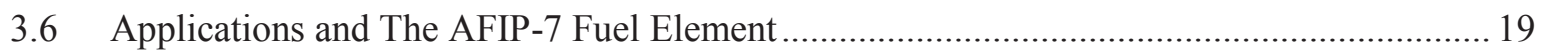

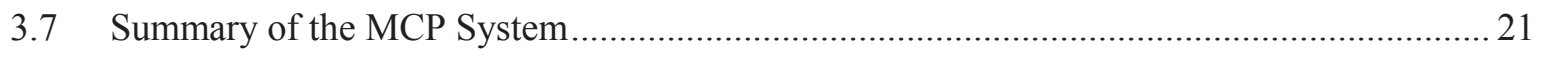

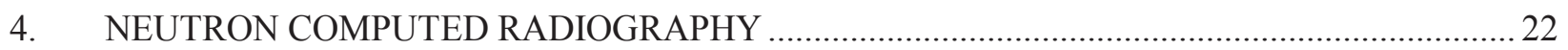

$4.1 \quad$ Photostimulable Storage Phosphor Imaging Plates............................................................ 23

4.2 Spatial Resolution of Transfer Method Neutron Radiography Techniques .......................... 24

4.2.1 Factors Affecting Spatial Resolution for Transfer Method Neutron

Radiography Techniques.................................................................................... 24

4.2.2 Comparison of the Spatial Resolution of Foil-Film Transfer Method Neutron

Radiography and Neutron Computed Radiography ............................................2 26

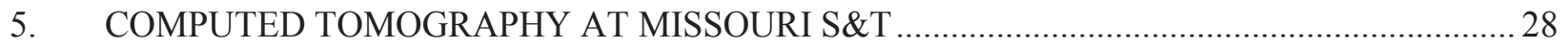

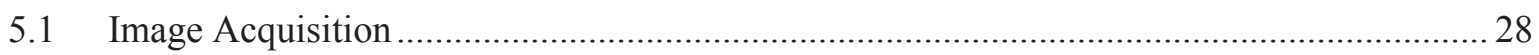

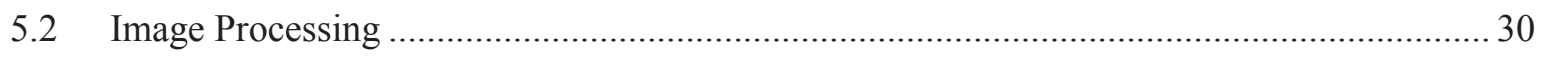

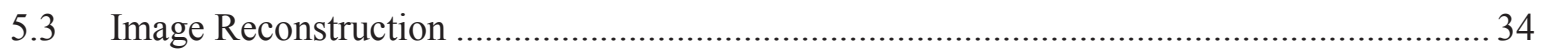

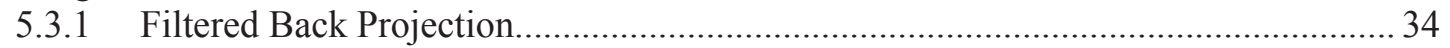

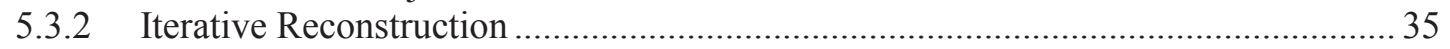

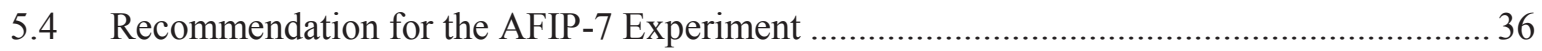

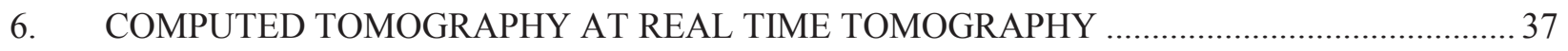

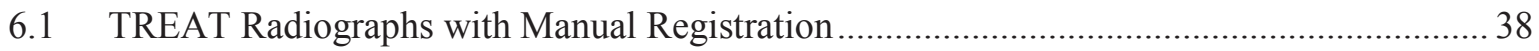

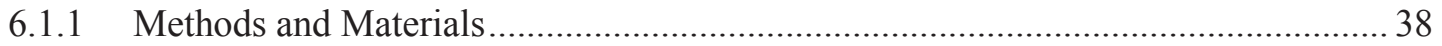

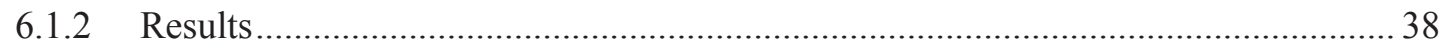

6.2 TREAT Radiographs with Semi Automated Registration ................................................ 41

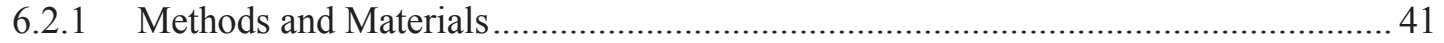

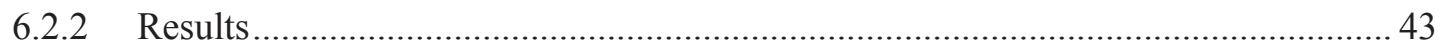

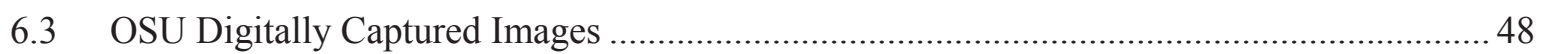

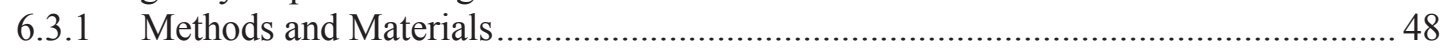

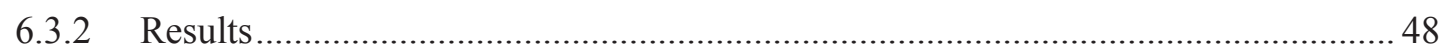

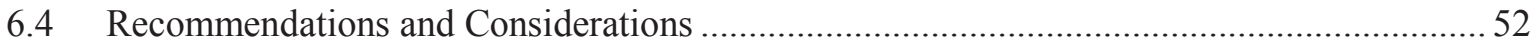

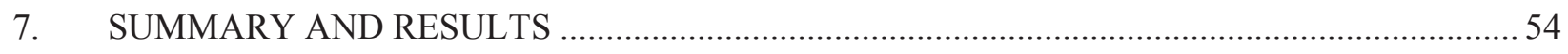

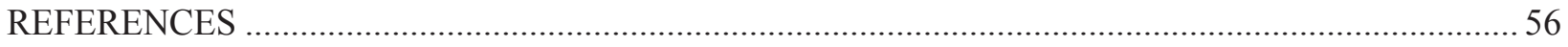




\section{LIST OF FIGURES}

Figure 2.1. Schematic of the indirect neutron radiography process.................................................... 2

Figure 2.2. Neutron radiography schematic with a foil cassette........................................................2

Figure 2.3. Foils loaded into the cassette for neutron radiography at NRAD .......................................... 3

Figure 2.4. Detector systems for neutron radiography by spatial and time resolution. .............................. 3

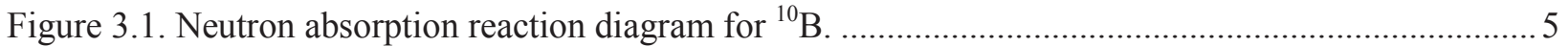

Figure 3.2. Schematic showing the interaction of a neutron with an MCP............................................ 6

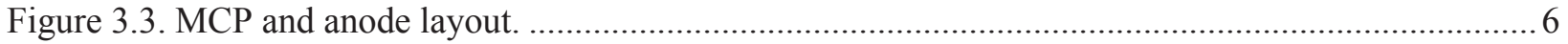

Figure 3.4. Effects of adjusting the threshold to achieve a flat detector response. ................................... 8

Figure 3.5. Gd-coated pinhole mask with $300 \mu \mathrm{m}$ diameter holes. ......................................................... 9

Figure 3.6. Uncorrected and corrected digital radiographs of a pinhole mask used for detector calibration. 10

Figure 3.7. Digital radiographs showing effect of contrast enhancement.............................................. 10

Figure 3.8. Digital radiographs showing the effect of a $0.02 \mathrm{R} / \mathrm{hr}$ at $1 \mathrm{~m}{ }^{137} \mathrm{Cs}$ source on the MCP detector.

Figure 3.9. Digital radiographs of a cadmium strip taken with a ${ }^{124} \mathrm{Sb}, 0.33 \mathrm{R} / \mathrm{hr}$ source near the detector.

Figure 3.10. Digital radiographs of a cadmium strip taken with a mixed $357 \mathrm{mCi}^{124} \mathrm{Sb}, 4.6 \mathrm{mCi}$

${ }^{122} \mathrm{Sb}$ source $5 \mathrm{~cm}$ the detector. 12

Figure 3.11. Schematic of the sensitivity indicator. .13

Figure 3.12. Schematic of the beam purity indicator. .14

Figure 3.13. Digital radiographs showing orthogonal views of the BPI............................................. 15

Figure 3.14. Digital radiographs of the SI showing effect of flat-field correction. .................................. 16

Figure 3.15. Digital radiograph of the SI after significant adjustment of the threshold settings............... 16

Figure 3.16. Orthogonal radiographs of the BPI and respective sinograms. .......................................... 17

Figure 3.17. Two-dimensional slices of a tomographic reconstruction of a BPI................................... 18

Figure 3.18. Three-dimensional representation of the tomographic reconstruction of the BPI................. 18

Figure 3.19. Orthogonal radiographs of AFIP-7 mock-up element. ....................................................... 19

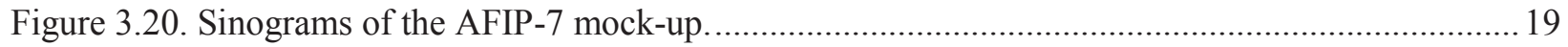

Figure 3.21. Tomographic slice of the simulated AFIP-7 fuel element..................................................20

Figure 4.1. Schematic of the neutron computed radiography process. ...................................................22

Figure 4.2. Qualitative schematic of an impinging neutron beam on a foil..........................................24

Figure 4.3. Spatial effects of the thickness of an activation foil for transfer method neutron

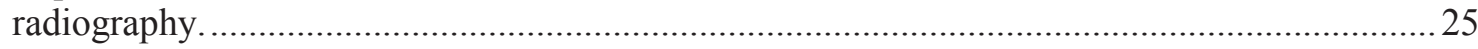

Figure 4.4. MTF of neutron radiography for both film and imaging plates...........................................22

Figure 5.1. Digitized film radiographs of the L07 fuel bundle from three different angles.....................22 


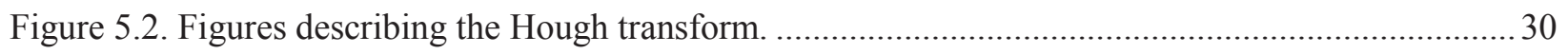

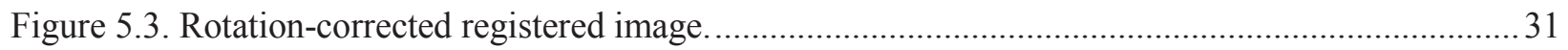

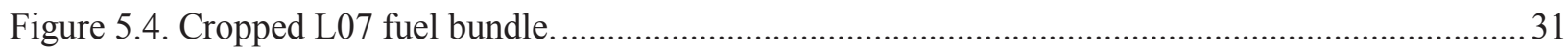

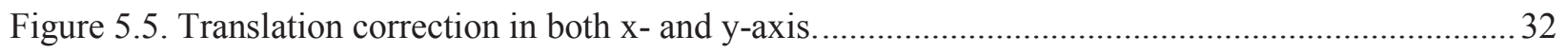

Figure 5.6. Noise reduction using alpha trimmed mean filtering. ........................................................ 33

Figure 5.7. Dynamic range corrected and cropped from bottom. ........................................................ 33

Figure 5.8. Reconstruction using FBP algorithm with a Hamming filter. ................................................. 34

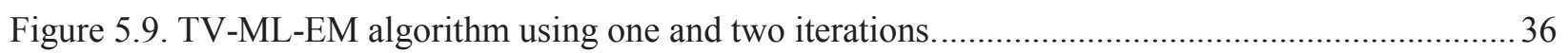

Figure 6.1. Twelve projection $26.4^{\circ}$ TREAT longitudinal reconstructions at three positions....................39

Figure 6.2. Distance measurements on TREAT longitudinal reconstruction with 12 projections

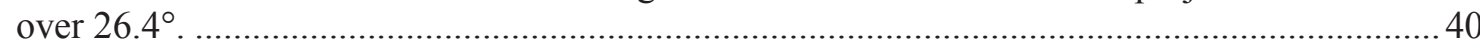

Figure 6.3. Transverse TREAT reconstructions from 76 projections at two different axial

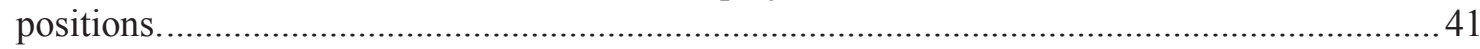

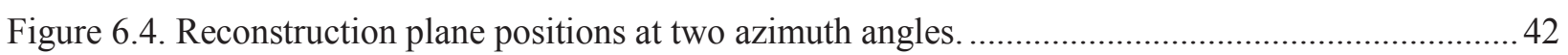

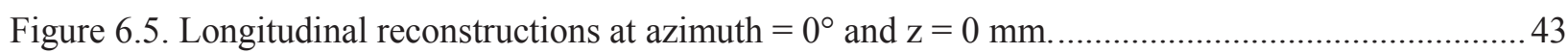

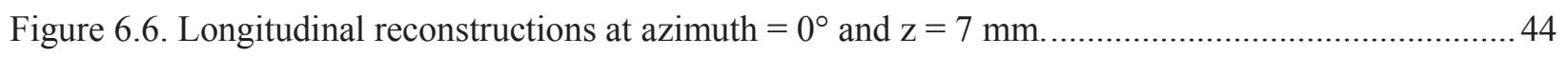

Figure 6.7. Longitudinal reconstructions at azimuth $=30^{\circ}$ and $\mathrm{z}=0 \mathrm{~mm}$........................................45

Figure 6.8. Longitudinal reconstructions at azimuth $=30^{\circ}$ and $\mathrm{z}=3.5 \mathrm{~mm}$....................................46

Figure 6.9. MCP detector and imaged plate assembly (a) and a diagram of image acquisition

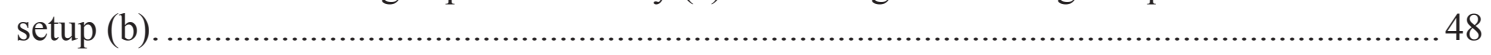

Figure 6.10. Reconstructions using the OSU projections. ............................................................ 49

Figure 6.11. Regions of interest and corresponding histograms from two orthogonal projection

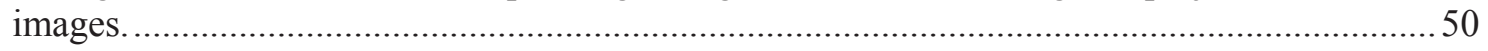

Figure 6.12. Projection image profile plots through the relatively high contrast plate holder....................51

Figure 6.13. Top and side views of a suggested fiducial marker arrangement to validate the

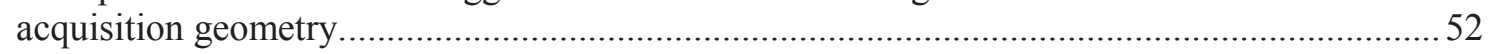

Figure 6.14. Illustration of image acquisition geometry for the AFIP-7 fuel element..............................53 


\section{LIST OF TABLES}

Table 3.1. Variable definitions for calculation of ASTM radiography facility parameters. ...................... 14

Table 3.2. Parameters to determine the ASTM radiography facility category. ....................................... 14

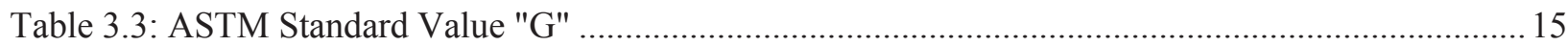

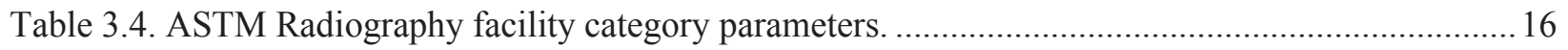

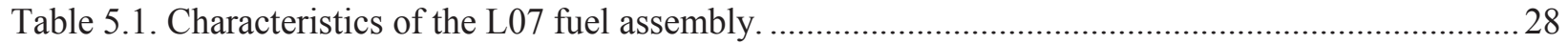

Table 5.2. Technical specifications for the Genesis NEO S60 scanner..................................................29

Table 6.1. Fuel rod actual and reconstruction measurements. .................................................................. 40

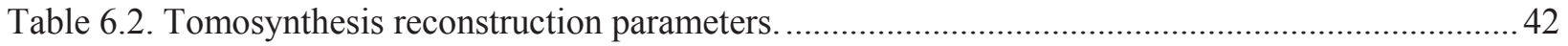

Table 6.3. Projection artifacts noted from OSU digital image acquisition. .............................................50 


\section{ACRONYMS}

2D/3D two-dimensional / three-dimensional

AFIP

ATR Full-size In center flux trap Position

ASTM

American Society for Testing and Materials

BPI

beam purity indicator

$\mathrm{CR}$

computed radiography

CT

computed tomography

ERS East Radiography Station

FBP

filtered back-projection

GTRI Global Threat Reduction Initiative

HEU highly-enriched uranium

HFEF Hot Fuel Examination Facility

IP imaging plate

IQI image quality indicator

INL Idaho National Laboratory

LET linear energy transfer

LEU low-enriched uranium

lp line pairs; sometimes referred to as "cycles" in digital imaging applications

MCP micro-channel plate or multi-channel plate

MST Missouri University of Science and Technology (or Missouri S\&T)

MTF modulation transfer function

NNSA National Nuclear Security Agency

NRAD Neutron Radiography (NRAD) Reactor Facility

NRF Neutron Radiography Facility at OSU

OD optical density

OSU Oregon State University

PSP photostimulable storage phosphor

PTFE polytetrafluoroethylene

RERTR Reduced Enrichment for Research and Test Reactor

ROI Region of Interest

RTT Real Time Tomography

SI sensitivity indicator

TREAT Transient Reactor Test Facility

TV-ML-EM total variation maximum likelihood expectation maximization 


\section{ABSTRACT}

This project seeks to assess the geometric stability of the U-Mo monolithic fuel system by evaluating the radiation-induced changes in the AFIP-7 experiment device. Neutron radiography and computed tomography (CT) provide valuable information about the post-irradiation condition of the fuel specimen. Tomographic reconstructions of the AFIP-7 fuel element will be analyzed to assess the geometric condition of the element after irradiation and provide information regarding the condition of the fuel, including gross geometric defects, bowing, twist, plate buckling, cracks, and other defects. The INL, in collaboration with Oregon State University (OSU), Missouri University of Science and Technology (Missouri S\&T), and Real Time Tomography (RTT), is developing advanced neutron detector systems and tomographic reconstruction techniques to evaluate the AFIP-7 fuel element. Neutron computed tomography using the current neutron radiography technique available at the Neutron Radiography reactor (NRAD) is impractical due to the long time and high cost to produce a set of images for tomographic reconstruction. Advanced neutron radiography systems such as the micro-channel plate (MCP) detector and neutron computed radiography (CR) may reduce the time and cost of acquiring images for neutron CT.

The MCP detector system tested at OSU and Missouri S\&T provides neutron radiographs and has lower gamma sensitivity compared to other digital acquisition image systems. However, some significant, but not prohibitive, challenges must be overcome to make its use for imaging nuclear fuel more practical. Images taken with the MCP require significant image processing to reduce distortions and correct for the dynamic detector response. Also, the small active area of the detector ( $\sim 30 \mathrm{~mm}$ diameter) requires the collection and combination of several images of a specimen, which may become time-consuming. The MCP is tested in low gamma dose environments, but should also be tested in the gamma field at the INL.

In addition to the MCP detector system, the INL is developing neutron computed radiography (CR), which uses the same foil transfer method currently employed at the NRAD, but replaces the film with a photostimulable storage phosphor imaging plate that is sensitive to the decay radiation from the activated transfer foils. Preliminary tests show that neutron CR provides lower, but comparable spatial resolution properties to film radiography images using the transfer method technique already employed at the NRAD. However, there are many factors that affect the spatial resolution of neutron CR that can be adjusted and possibly provide spatial resolution properties that exceed film radiography. The reduction in cost of neutron CR may be significant, which would make neutron imaging more appealing to programs that would benefit from this rare and valuable capability.

Because transfer method neutron radiography is expensive and time-consuming, acquisition of a full set of radiographs for tomographic reconstruction is impractical. The INL is developing tomographic reconstruction techniques that require only a small set of radiographs of the AFIP-7 fuel element. These tomosynthesis techniques are tested using archived radiographs of TREAT experiments for CT as a proof of principle. These same techniques are applied to radiographs taken with an MCP detector of a mock-up of the AFIP-7 geometry.

Acquisition angle should at least match the curvature of AFIP-7 plates. Lateral (in-plane) reconstruction resolution will be best when the projection angles include views at or near the desired reconstruction view angles. The ends of the fuel plates are angled at $\pm 17.7^{\circ}$ relative to the center of the plate. Increasing the number of projections is desirable, but given the constraint of minimizing the number of projections, the projection acquisition angle should also span at least $\pm 17.7^{\circ}$ to optimize the lateral resolution of the angled reconstruction planes used to visualize the gaps at the ends of the fuel plates. If possible, enough projections should be used within the initial acquisition angle of $35.4^{\circ}\left( \pm 17.7^{\circ}\right)$ to bring the projection angular sampling closer to $1^{\circ}$. Further testing will determine a more specific recommendation for the number and angular distribution of neutron radiographs that will produce the highest quality tomographic reconstruction of the AFIP-7 fuel element. 


\section{INTRODUCTION}

The Convert branch of the National Nuclear Security Administration (NNSA) Global Threat Reduction Initiative (GTRI), is working to develop high uranium density fuels for research and test reactors. This fuel is intended to enable conversion from highly enriched uranium (HEU) based fuel to low enriched uranium (LEU) based fuel for high performance research reactors. There are five U.S. reactors that fall under the high performance research reactor category, including: the Massachusetts Institute of Technology Reactor, the National Bureau of Standards Reactor, the Missouri University Research Reactor, the Advanced Test Reactor, and the High Flux Isotope Reactor.

Two fuel designs are being developed that use a uranium molybdenum alloy fuel phase, a dispersion and monolithic version. The ATR Full-size In center flux trap Position (AFIP-7) experiment consists of four monolithic foil type fuel plates. This project seeks to assess the geometric stability of the U-Mo monolithic fuel system and evaluate the radiation effects in the AFIP-7 experiment device. Neutron radiography and computed tomography $(\mathrm{CT})$ provide valuable and comprehensive information about the post-irradiation condition of the fuel specimen. Tomographic reconstructions of the AFIP-7 fuel element will be analyzed to assess the geometric condition of the element after irradiation and provide information regarding the condition of the fuel, including gross geometric defects, bowing, twist, plate buckling, cracks, and other defects. The INL, in collaboration with OSU, Missouri S\&T, and Real Time Tomography, is developing advanced neutron detector systems and tomographic reconstruction techniques to evaluate the AFIP-7 fuel element.

Neutron computed tomography using the current neutron radiography technique available at NRAD is impractical due to the long time and high cost required to provide a set of images for tomographic reconstruction. Advanced neutron radiography systems, such as the MCP detector and neutron CR, may reduce the time and cost of acquiring images for neutron $\mathrm{CT}$. The current neutron radiography capabilities at the NRAD are discussed in Section 2, followed by a discussion of advanced neutron radiography systems under development. The development of the MCP detector system at OSU is discussed in detail in Section 3, followed by a discussion of neutron CR in Section 4.

In addition to developing ways to reducing the time and cost of producing neutron radiographs, the INL is seeking to reduce the number of radiographs required by developing tomographic reconstruction techniques that would need only a small set of radiographs of the AFIP-7 fuel element to produce a highquality tomographic reconstruction. The development of this technique is discussed in detail in Section 5. 


\section{CURRENT NEUTRON IMAGING CAPABILITIES AT THE NRAD}

Neutron radiography at the NRAD facility is performed using the indirect foil-film transfer technique. Indirect neutron radiography methods involve the use of an intermediate detector, which produces an image upon subsequent processing. In the foil-film transfer technique, a transfer foil (e.g. dysprosium, indium, gold) serves as the intermediate detector by recording the image as a pattern of activation of the foil material. ${ }^{1}$ The transfer method requires more time than some direct radiography techniques, but it offers the advantage that the film is not present in the neutron beam, thus offering complete discrimination against gamma ray fogging. The transfer method can thus be employed to image highlyradioactive objects, which give off significant gamma radiation that would fog the film in the direct method. ${ }^{2-4}$

Figure 2.1 shows a schematic of the indirect radiography process. A transfer foil placed behind the object becomes activated as it absorbs neutrons that penetrate through the object. After irradiation, the activated foil is taken to a dark-room and placed in contact with an x-ray film. The decay radiation (primarily beta radiation) exposes the film according to the activity pattern of the activated foil. Subsequent development of the film produces a neutron image of the object.

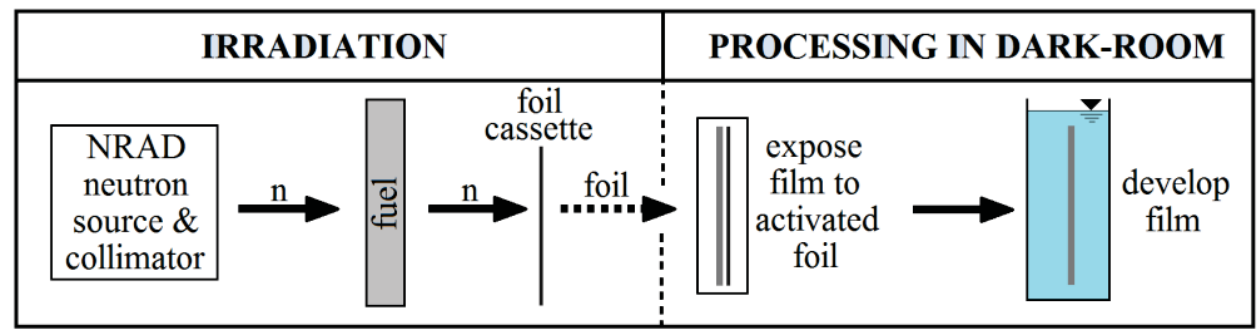

Figure 2.1. Schematic of the indirect neutron radiography process.

At the NRAD, activation foils are loaded into cassette and placed behind the specimen, often a nuclear fuel experiment, as shown in Figure 2.2. The neutron flux before reaching the specimen, $\phi_{0}$, is attenuated by the specimen according to the thickness and macroscopic attenuation cross-section of the materials in the specimen. The foils in the cassette are activated by the pattern of the neutron flux that passes through the specimen, $\phi(\mathrm{x})$.

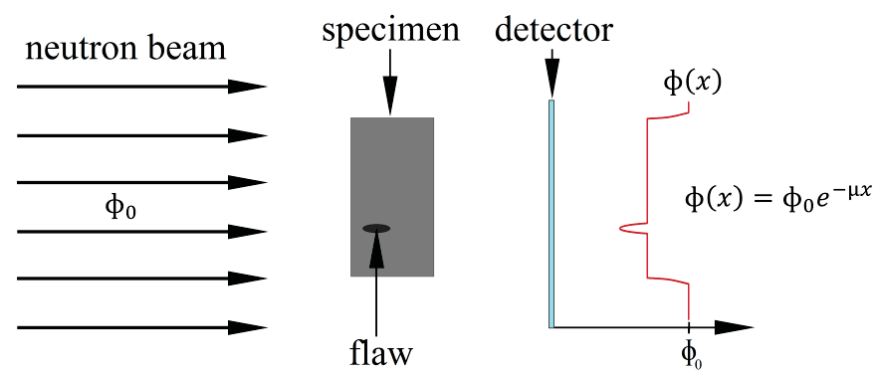

Figure 2.2. Neutron radiography schematic with a foil cassette.

A dysprosium transfer foil can be combined with a cadmium foil and indium foils for simultaneous dualimage radiography. ${ }^{4,5}$ At the NRAD, the foil cassette is loaded such that the first foil behind the object is dysprosium, followed by cadmium, then indium, as shown in Figure 2.3. ${ }^{5}$ The cadmium strongly absorbs thermal neutrons, exposing the indium foil to only epithermal and fast neutrons. Thus, both epithermal and thermal neutron images can be produced with a single neutron exposure. ${ }^{5}$ 


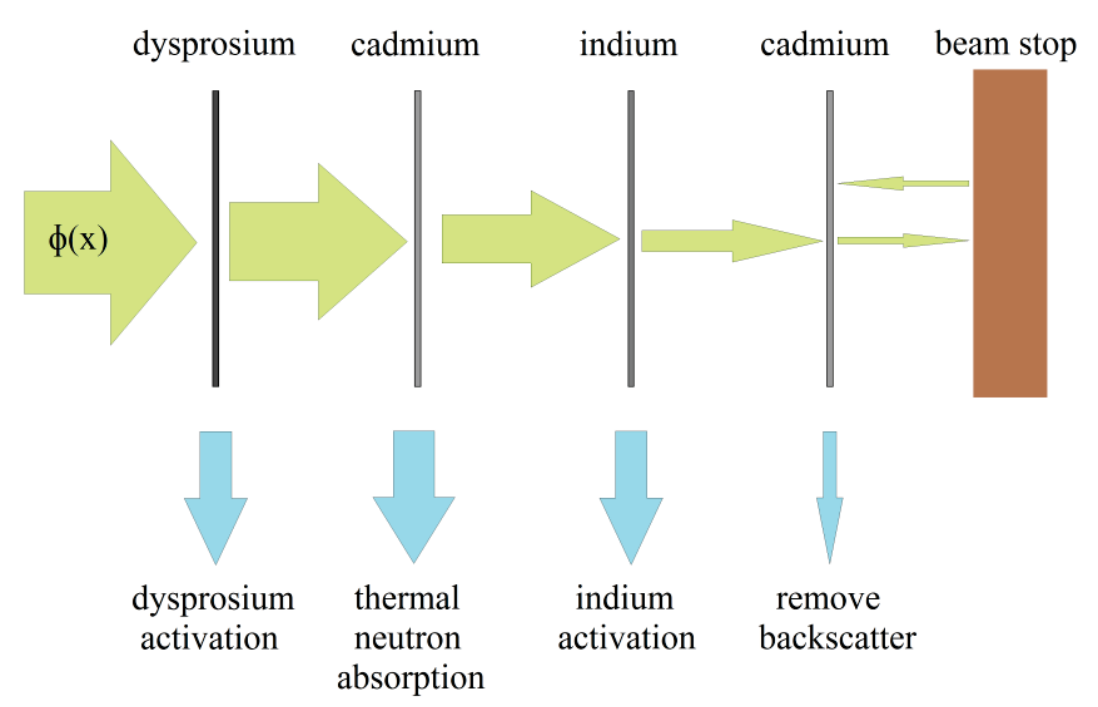

Figure 2.3. Foils loaded into the cassette for neutron radiography at NRAD.

Indium has a large activation resonance at $1.46 \mathrm{eV}$, and has been widely used to detect these higher energy neutrons. ${ }^{6}$ Other potential useful epithermal detectors include gold with a resonance at $4.9 \mathrm{eV}$ and tungsten with a resonance at $18.8 \mathrm{eV}{ }^{6}$ Epithermal imaging techniques can offer information that is not available from thermal neutron radiography of nuclear fuels because higher neutron energies provide greater penetration through ${ }^{235} \mathrm{U}$-enriched and plutonium-containing materials. ${ }^{6}$

Neutron tomography typically requires a set of multiple images taken over $180^{\circ}$ of a specimen in small, perhaps in $1^{\circ}$ increments. Acquiring this many images using the transfer method at the NRAD is prohibitively time-consuming and expensive. Other neutron radiography systems are available that would decrease the time required to produce an image. Figure 2.4 describes the spatial and time resolution of multiple detector systems for neutron radiography.

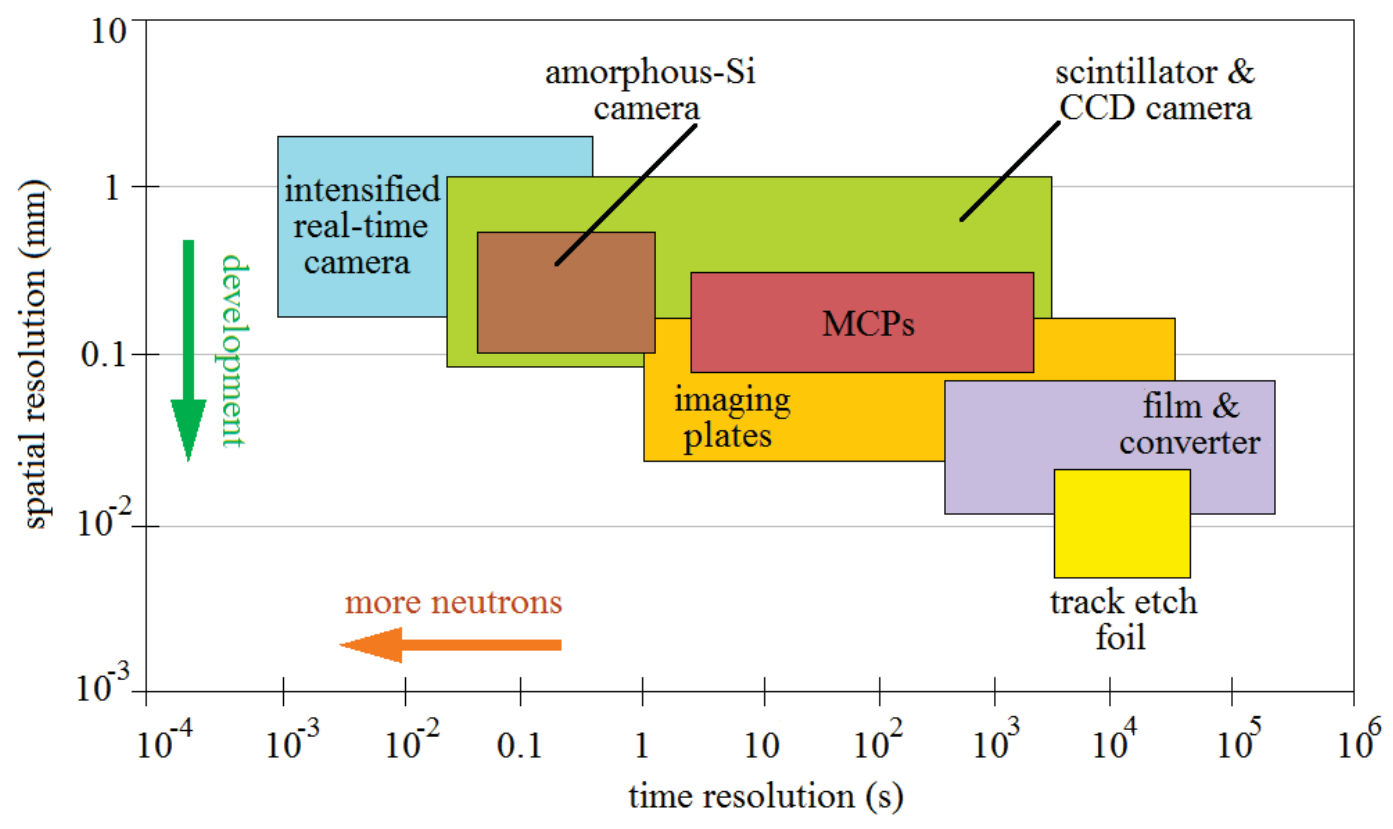

Figure 2.4. Detector systems for neutron radiography by spatial and time resolution. 
Increasing the neutron beam flux typically decreases the time required to produce an image. This is true for real-time digital systems, but for systems that require additional processing after exposure (e.g. foilfilm transfer method), the beam exposure time is often not the limiting factor in the time required to produce an image. While foil-film transfer technique neutron radiography provides relatively high spatial resolution, the time required to produce a radiograph with this technique is two to three orders of magnitude greater compared to modern digital systems. ${ }^{7}$ The longer exposure time also makes tomographic reconstruction using multiple foil exposures prohibitively expensive. ${ }^{7}$

Detector systems for performing neutron radiography at the NRAD facility will experience high gamma doses from highly radioactive specimens, and thus must either have low sensitivity to gamma radiation (e.g. MCPs) or be totally insensitive to gamma radiation (e.g. indirect foil-film transfer method or computed radiography using image plates).

This project seeks to perform computed tomography with a set of neutron radiographs, and a significant portion of this project focuses on testing advanced neutron radiography systems that can produce quality neutron radiographs in less time, which simultaneously reduces cost. The following section describes the development and testing of MCP systems, followed by a section that describes the development and characterization of computed radiography systems. 


\section{DEVELOPMENT OF MULTI-CHANNEL PLATE IMAGING SYSTEMS}

The foil-film transfer technique currently employed at the INL takes a relatively long time to produce an image compared to some modern neutron imaging systems, and requires significant personnel time and cost. The INL has been assessing advanced neutron radiography techniques and detectors that may be able to produce quality neutron radiographs of nuclear fuel with reduced time and cost. Multi-channel plate detectors may offer significantly reduced (nearly real-time) image acquisition time and has reduced gamma sensitivity, which may make it possible to take images of nuclear fuel using MCP detector systems. The following section describes the fundamentals of MCP systems, followed by sections describing the detector electronics, the gamma sensitivity of the MCP detector, determination of the ASTM radiographic category for the detector with OSU's neutron beam. Computed tomography using the MCP detector and application to the AFIP-7 fuel element are also examined.

\subsection{Fundamentals of Multi-Channel Plate Neutron Imaging Systems}

MCP imaging detectors with high spatial resolution and timing accuracy are already widely used for detection of electrons and ions, as well as UV and x-rays. Neutron sensitive MCPs have been recently developed that can provide neutron radiographic capabilities with high spatial resolution. The MCP detector includes a thin glass wafer with micron-size diameter channels in a hexagonal array. Ionizing radiation (e.g. X-rays, UV-rays, electrons) liberates charged particles from interactions with the MCP bulk material. When a high-voltage difference is applied to the MCP, the charged particles produce an electron cascade down the channels, amplifying the initial charged particles into a detectable signal. The electron signal is read by an anode at the back-end of the MCP, which produces a location and time-stamp for each signal, and software uses the locations and time-stamps to produce a 2D image.

Neutrons are not a directly-ionizing form of radiation, but are considered indirectly-ionizing since they can interact with nuclei to produce ionizing radiation, often in the form of prompt gamma radiation, prompt beta radiation, large charged particles (i.e. split nuclei), and/or free electrons. Materials with a high neutron absorption cross-section added to the MCP glass make the MCP neutron sensitive. Specifically, compounds containing ${ }^{10} \mathrm{~B}$ (boron oxide, $\mathrm{B}_{2} \mathrm{O}_{3}$ ) and sometimes gadolinium (gadolinium oxide, $\mathrm{Gd}_{2} \mathrm{O}_{3}$ ) are added to the MCP glass to convert neutrons into charged particles through a ${ }^{10} \mathrm{~B}(\mathrm{n}, \alpha)^{7} \mathrm{Li}$ reaction, illustrated in Figure $3.1 .^{8}$ This reaction produces ${ }^{7} \mathrm{Li}(1.01 \mathrm{MeV})$ and a recoil $\alpha$ particle $(1.8$ $\mathrm{MeV})$ with a branching ratio of $6 \%$, or ${ }^{7} \mathrm{Li}(0.84 \mathrm{MeV})$ and an $\alpha$ particle $(1.47 \mathrm{MeV})$ and a gamma ray $(0.48 \mathrm{MeV})$ with a branching ratio of $94 \% .{ }^{9}$

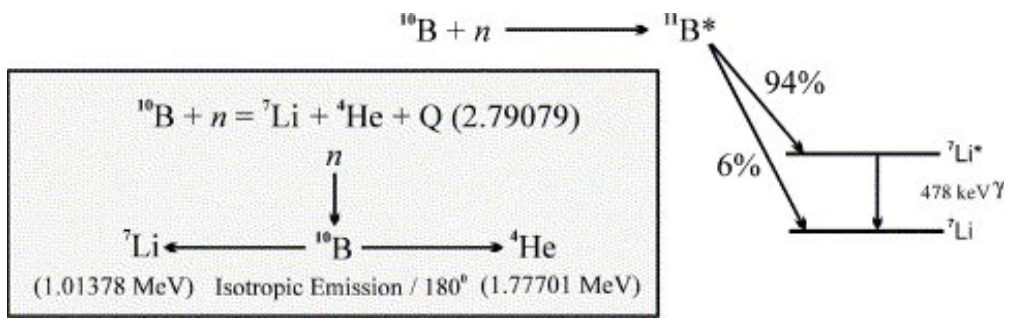

Figure 3.1. Neutron absorption reaction diagram for ${ }^{10} \mathrm{~B}$.

Figure 3.2 illustrates the neutron interaction with the MCP. The reaction products, primarily the $\alpha$ particle, create secondary electrons directly in MCP channels through ionizations with the channel walls, which in turn initiate an electron avalanche and a subsequent strong output pulse. The $\alpha$ particle has a very short range, approximately $3.5 \mu \mathrm{m}$ within the MCP glass, and a very high probability of initiating an electron avalanche, along with the ${ }^{7} \mathrm{Li}$ which may be fully absorbed within the glass walls due to its higher linear energy transfer (LET). 


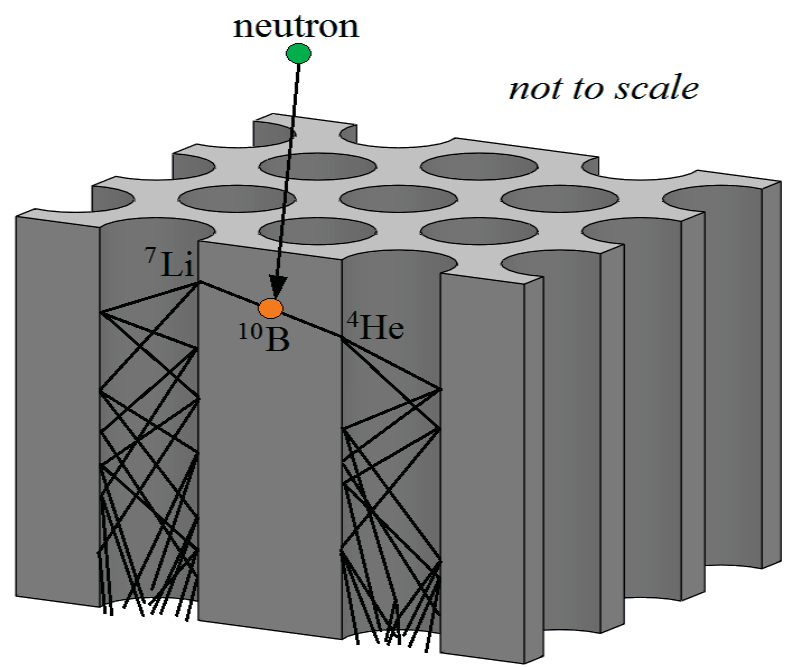

Figure 3.2. Schematic showing the interaction of a neutron with an MCP.

Multiple MCPs can be stacked to increase neutron capture efficiency and amplification of the signal to the anode. The neutron reactions primarily occur in the forward most MCP, where this MCP provides a gain of $10^{6}$ to $10^{7}$. Figure 3.3 illustrates the collection of an electron signal at the anode. ${ }^{9}$ A delay line anode then captures the electron cloud from the MCP stack and integrates the electrons as a charge. This allows for the signal to be interpreted by user controlled electronics and an event location is then calculated, producing a spatial resolution of $\sim 9 \mu \mathrm{m}$. The track length of the $\alpha$ particle, $3.5 \mu \mathrm{m}$, can be in any direction, giving a spatial resolution of $\sim 7 \mu \mathrm{m}$. Summing the two values gives a theoretical maximum neutron event spatial resolution of approximately $15 \mu \mathrm{m}$.

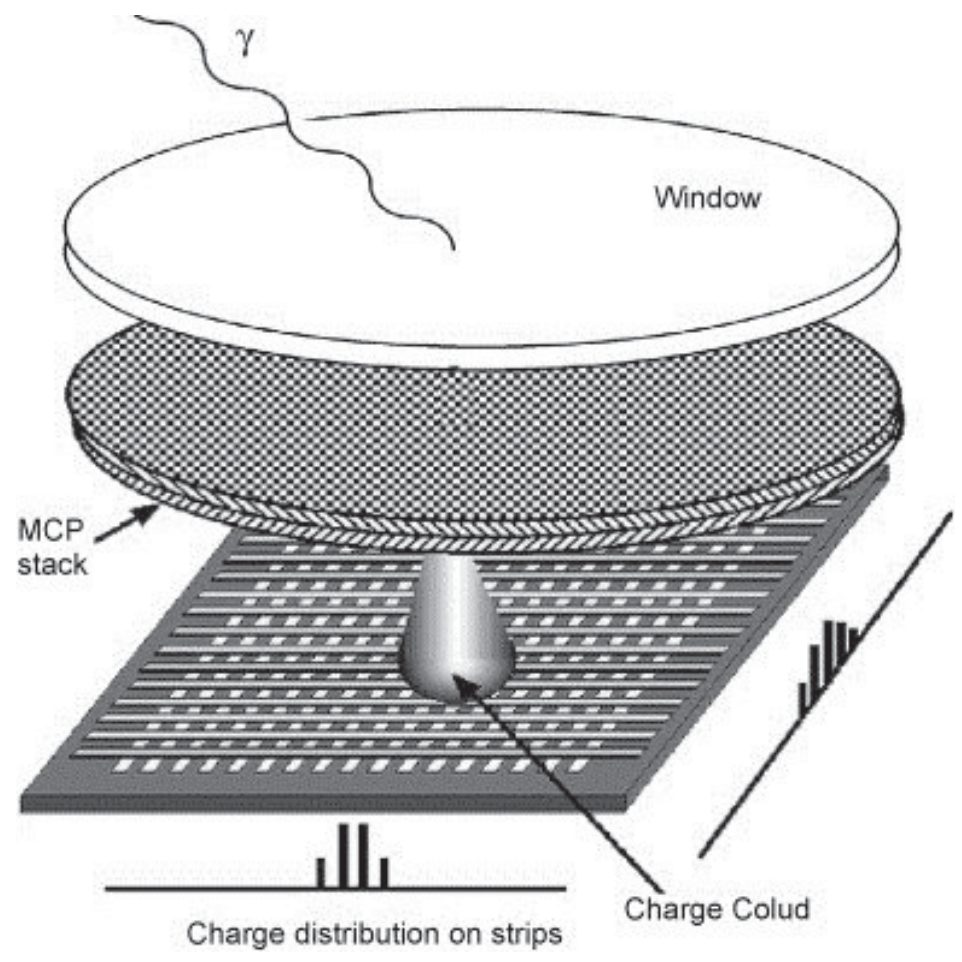

Figure 3.3. MCP and anode layout. 
The neutron detection efficiency, $\mathrm{Q}_{\mathrm{n}}$, of a ${ }^{10} \mathrm{~B}$-doped MCP detector is given by the product of three terms, $\mathrm{Q}_{\mathrm{n}}=\mathrm{P} 1 \times \mathrm{P} 2 \times \mathrm{P} 3$, where $\mathrm{P} 1$ is the fraction of incident neutrons absorbed by the MCP structure, $\mathrm{P} 2$ is the fraction of those interactions that generate an electron avalanche within an MCP channel, and P3 is the fraction of avalanches registered by the electronics. Due to the sensitive nature of the MCP, a neutron interaction always yields an electron pulse, resulting in a $\mathrm{P} 2 \approx 1$. Therefore, the primary detection process consists of the multiplication of the terms $\mathrm{P} 1$ and $\mathrm{P} 3{ }^{8}$ While the final $\mathrm{Q}_{\mathrm{n}}$ is measured, $\mathrm{P} 1$ and $\mathrm{P} 3$ are not individually measured in this study. The probability of absorption (P1) and the probability of initiating an avalanche (P2) are judiciously calculated in a separate study. ${ }^{8}$

The value of P3 depends on the MCP detector operation and user control. This control consists of using appropriate bias voltages, configuring MCPs in a proper configuration, as well as the threshold range of the pulse readout electronics. Optimizing these settings is possible because the system is a high speed detection system the effects of the adjustments are shown instantly. Assuming that the P3 value has been optimized, the product of $\mathrm{P} 1$ and $\mathrm{P} 2$ is determined by first calculating the rate of neutrons incident on the detector. This is calculated in the equation below by multiplying the neutron flux, with the active area of the detector.

$$
4.6 \times 10^{5} \frac{n}{\mathrm{~cm}^{2} \mathrm{~s}} \times \pi(1.25 \mathrm{~cm})^{2}=1.8 \times 10^{6} \frac{n}{\mathrm{~s}}
$$

Comparing the rate of neutrons incident at the detector to the maximum count rate achieved after the removal of noise, approximately $2.5 \times 10^{5} \mathrm{n} / \mathrm{s}$, the product $\mathrm{P} 1$ and $\mathrm{P} 3$, and therefore total detector efficiency, is approximately $14 \%$.

$$
\mathrm{Q}_{\mathrm{n}}=\mathrm{P} 1 \times \mathrm{P} 2 \times \mathrm{P} 3=\frac{2.5 \times 10^{5} \frac{n}{\mathrm{~s}}}{1.8 \times 10^{6} \frac{n}{\mathrm{~s}}} \times 100=14 \%
$$

\subsection{Configuring the Detector Electronics}

The electronics have a significant impact with MCP detector systems. The main power supply sets the bias voltage across the detector plates, and a bias voltage of $1800 \mathrm{~V}$ and $2900 \mathrm{~V}$ applied to the back and front plates, respectively, produces a noticeable signal from the detector. At this point, with all of the system thresholds minimized to allow for all events, the bias should be increased in small increments of $20 \mathrm{~V}$ without exceeding a voltage difference between the two plates of more than $1200 \mathrm{~V}$ or exceeding $3200 \mathrm{~V}$ on either plate, until the event count rate is optimized. The operator then visually adjusts the thresholds and timing within the preamplifier, constant fraction discriminator, and time delay card. Figure 3.4 shows the effects of varying thresholds, where the color gradient represents registered counts at that location. The operator adjusts the thresholds to reduce gradients in the detector response to achieve a detector response that is as flat as possible. 


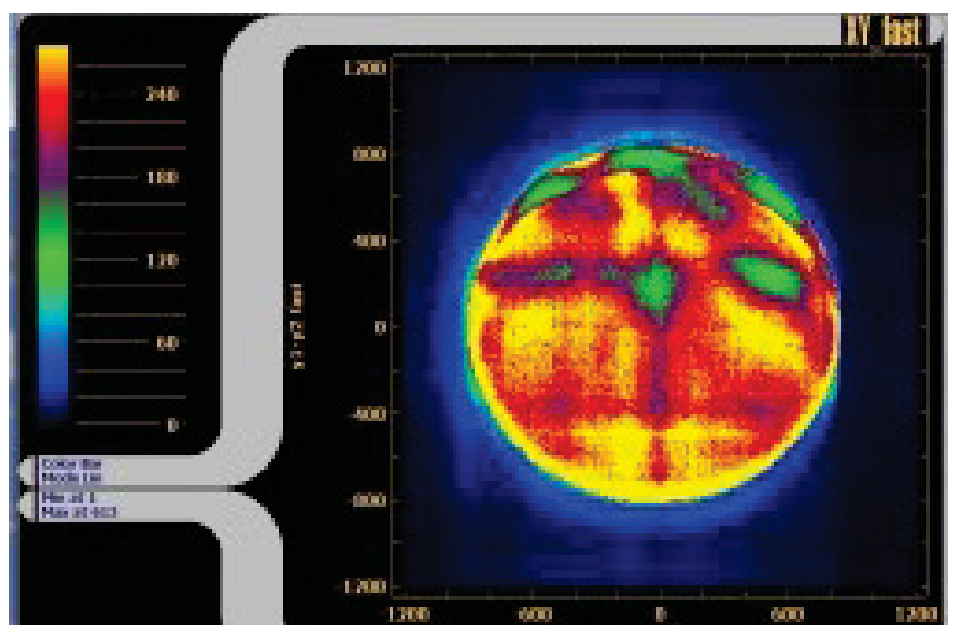

a) Detector response with poor threshold settings.

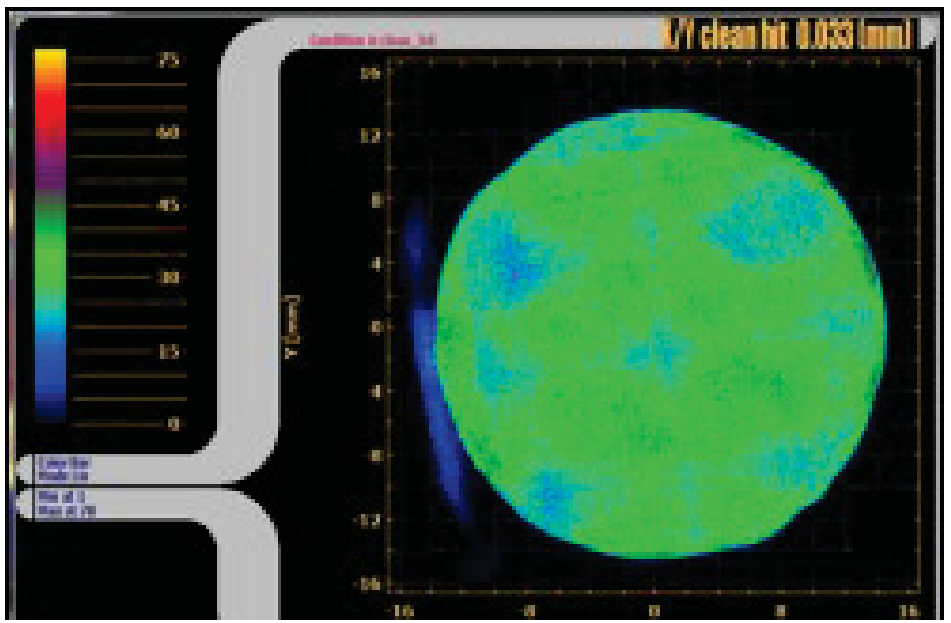

b) Detector response with improved threshold settings.

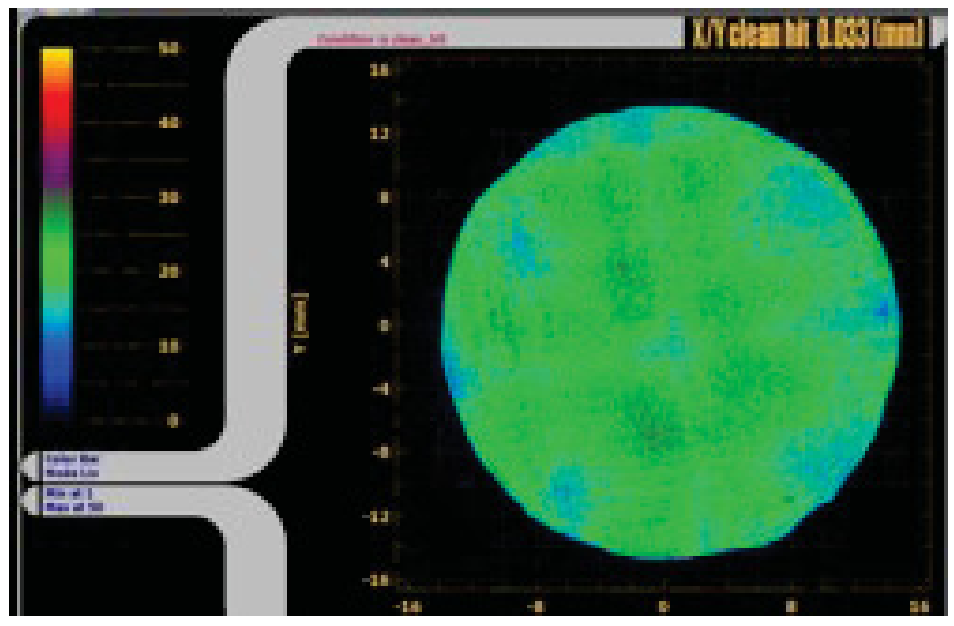

c) Detector response with a relatively flat response.

Figure 3.4. Effects of adjusting the threshold to achieve a flat detector response. 
With a relatively uniform image response from the neutron beam, an oscilloscope is used to adjust each timing and threshold value to fall just above the level of noise. This allows for multi-event counting by making it possible to distinguish each pulse as separate events. Pulse pileups are not a concern in this work because the neutron flux and the lower detector efficiency result in count rates lower than the 1.0 $\mathrm{MHz}$ maximum count rate of the detector. Some remaining non-uniformity within the image can be eliminated through image processing.

Off-gassing of adsorbed gasses in the micro-channels during initial operation produces changes in vacuum pressure and temperature, which cause small deviations within the timing and bias voltage. The pressure typically stabilizes after about an hour of operation in the neutron beam. However, until the detector response stabilizes, each image requires a separate threshold adjustment. Deviations in these radiographs are corrected by post-collection processing by taking an image of the beam without any specimens (i.e. a flat-field, background, or "light" image) and normalizing radiographs to remove the detector response to the neutron beam. This dynamic system requires either constant adjustment of the thresholds to maintain consistency or each individual image must have its own flat-field image for normalization. A combination of these two methods has been the most practical way to collect large numbers of radiographs in a single time frame. The dynamic nature of the detector may also be minimized with UV scrubbing prior to operation, which removes adsorbed gasses within the MCP channels by producing electron cascades with a UV light source instead of neutrons.

Unprocessed digital images produced by the MCP detector contain significant distortions caused by detector geometry, electronic timing, and other factors. These distortions are corrected by using an image of a pinhole mask with known dimensions (i.e. known spacing between the pinholes) to create a correction algorithm by comparing a distorted image of the pinhole grid to the known dimensions. The first pinhole grid used in this work is an aluminum plate coated with gadolinium oxide $\left(\mathrm{Gd}_{2} \mathrm{O}_{3}\right)$ with a series of $300 \mu \mathrm{m}$ diameter holes drilled through the mask in a $1 \mathrm{~mm}$ square array. A digital radiograph of this mask is shown in Figure 3.5. Some of the distortion of the detector is visible in Figure 3.5 as a departure from the perfectly square array of pinholes.

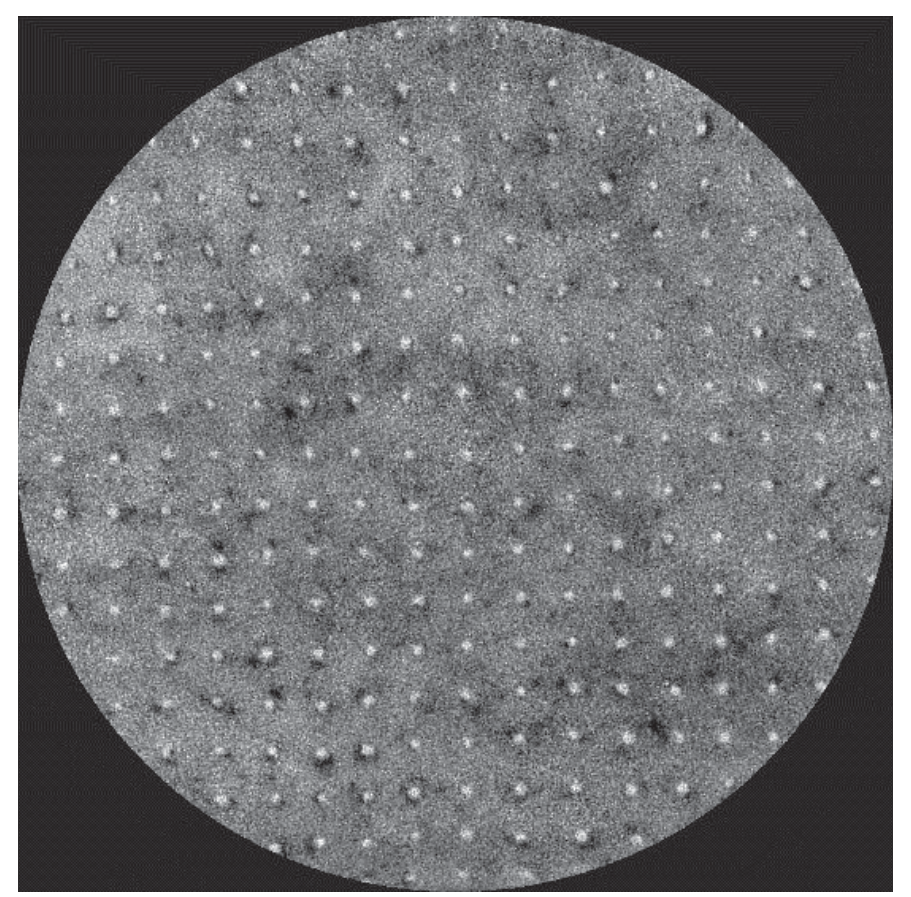

Figure 3.5. Gd-coated pinhole mask with $300 \mu \mathrm{m}$ diameter holes. 
The pinhole pitch $(1 \mathrm{~mm})$ of this first mask is relatively large and produces a coarse correction algorithm. In order to improve the correction algorithm on the edges of the image, where the distortion is most prominent, a digital radiograph is taken of a second pinhole mask with reduced scale. This mask is made of a gadolinium plate with $250 \mu \mathrm{m}$ diameter holes in a $1 \mathrm{~mm}$ square array. This mask was used to create an algorithm to correct future images and allow for acquisition of accurate dimensional measurements. Figure 3.6 shows a digital radiograph of this pinhole mask both before and after processing to remove the distortion. After application of the correction algorithm, the digital image of the pinhole grid shows no noticeable distortion (Figure 3.6b).

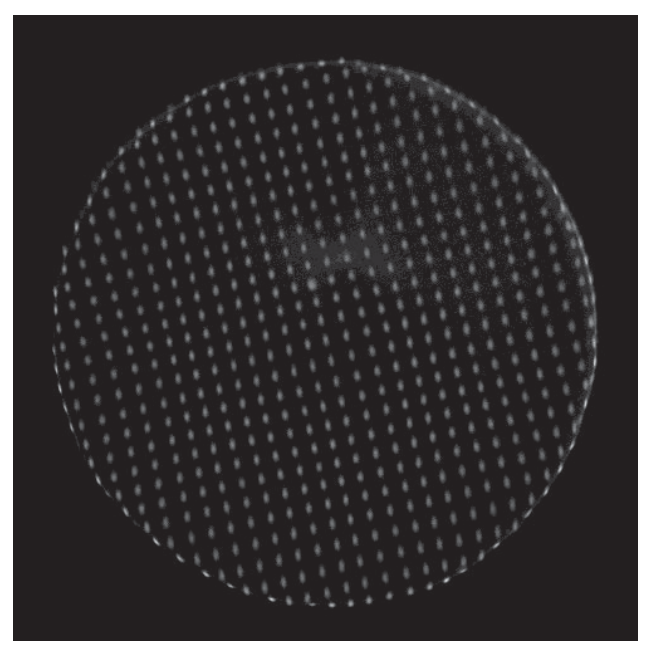

a) Uncorrected radiograph of a pinhole mask.

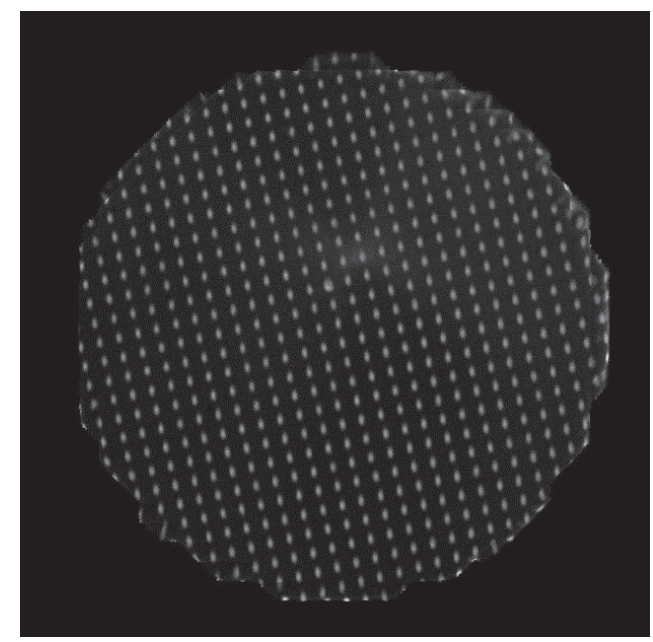

b) Corrected radiograph of a pinhole mask.

Figure 3.6. Uncorrected and corrected digital radiographs of a pinhole mask used for detector calibration.

Another image processing technique employed in this work normalizes and enhances contrast of a set of images. Contrast enhancement and utilization of increased dynamic range improves the ability to distinguish small features in a specimen. A set of images acquired for tomographic reconstruction should be processed prior to reconstruction to enhance the contrast of the images, which will lead to a more defined tomographic reconstruction with more distinguishable features. Figure 3.7 shows digital radiographs both before and after contrast enhancement.

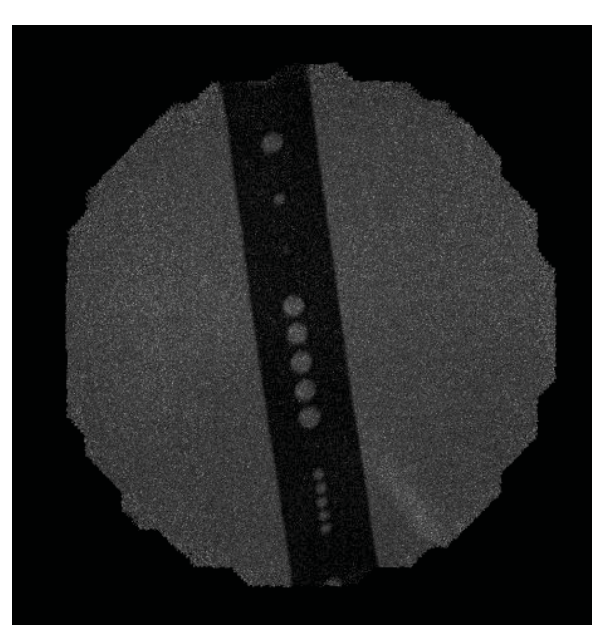

a) Radiograph prior to contrast enhancement.

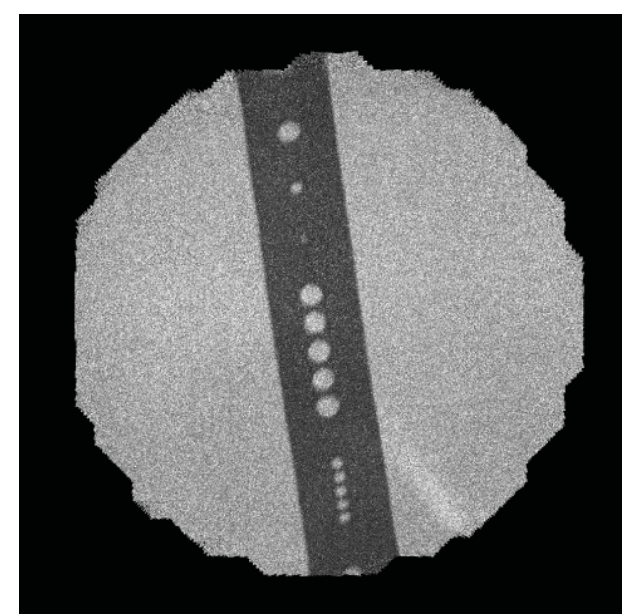

b) Radiograph after contrast enhancement.

Figure 3.7. Digital radiographs showing effect of contrast enhancement. 


\subsection{Gamma Sensitivity of the Multi-Channel Plate Detector}

A significant factor affecting neutron radiography is the gamma sensitivity of the detector system. A neutron beam at a nuclear reactor contains some gamma radiation from the reactor. While filters can reduce the gamma content of the neutron beam, it can never be completely removed. Gamma-sensitivity is also a major issue for neutron imaging of radioactive specimens such as nuclear fuel. Some neutron imaging detectors such as x-ray film and storage phosphor plates are easily overexposed in high gamma fields and therefore are used only with indirect imaging techniques. The MCP detector is much more sensitive to neutrons than to gamma radiation (or other low-LET ionizing radiation).

In order to determine how sensitive the MCP detector is to gamma radiation, the detector is exposed to gamma sources in addition to the inherent gamma radiation from the reactor beam. The first test uses a $0.2 \mathrm{R} / \mathrm{hr}$ at $1 \mathrm{~m}{ }^{137} \mathrm{Cs}$ source that emits $0.667 \mathrm{MeV}$ gamma radiation. Figure 3.8 shows both raw and flatfield corrected digital radiographs taken of a cadmium strip with drilled holes with the ${ }^{137} \mathrm{Cs}$ source both near $(10 \mathrm{~cm})$ and away $(1 \mathrm{~m})$ from the detector. While the uncorrected radiographs show a minor response to the presence of the gamma source (comparing Figure 3.8a to Figure 3.8b), flat-field correction adequately removes the effect of the gamma source (comparing Figure $3.8 \mathrm{c}$ to Figure $3.8 \mathrm{~d}$ ).

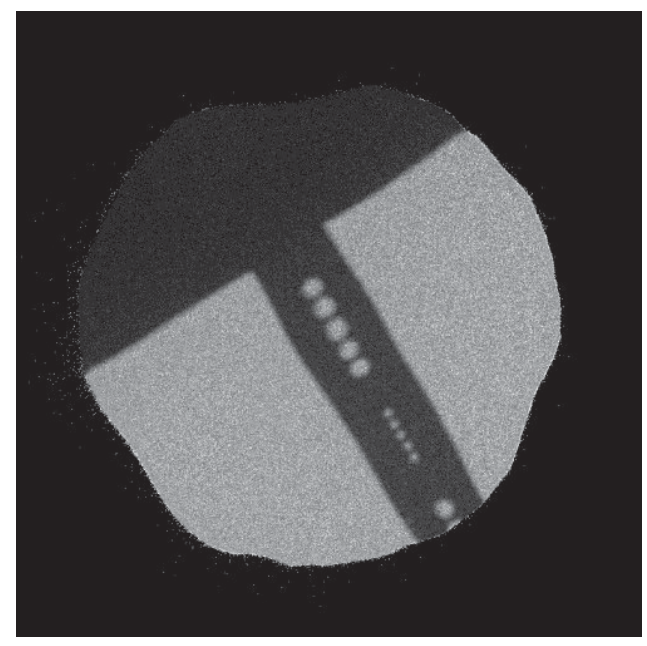

a) Radiograph with source at $1 \mathrm{~m}$.

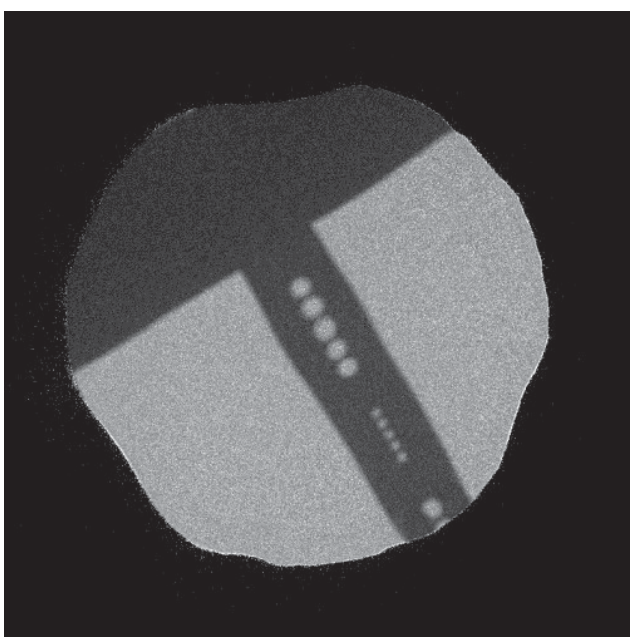

c) Flat-field corrected radiograph with source at $1 \mathrm{~m}$.

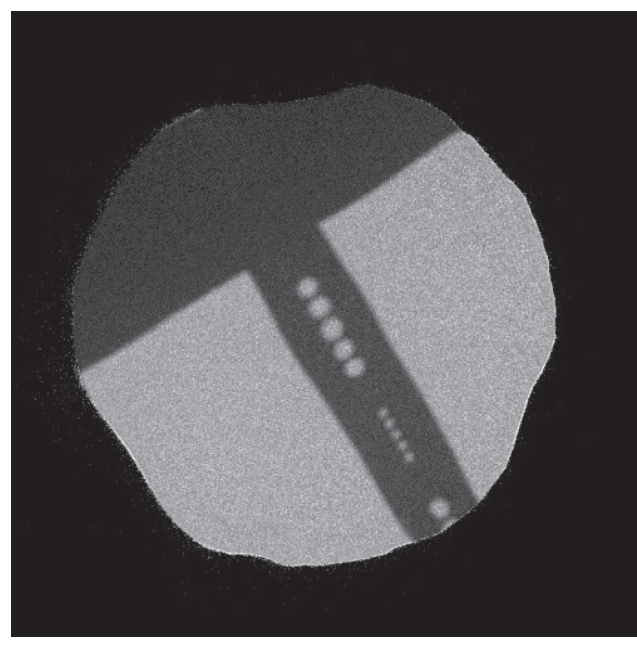

b) Radiograph with source at $10 \mathrm{~cm}$.

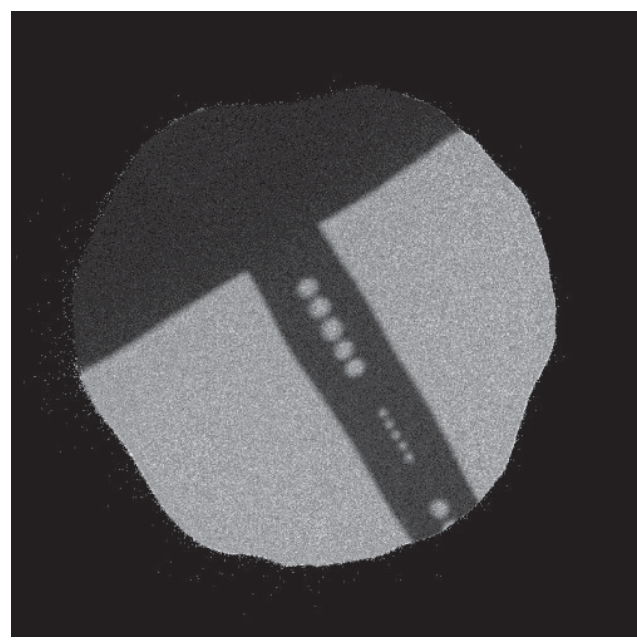

d) Flat-field corrected radiograph with source at $0.1 \mathrm{~m}$.

Figure 3.8. Digital radiographs showing the effect of a $0.02 \mathrm{R} / \mathrm{hr}$ at $1 \mathrm{~m}{ }^{137} \mathrm{Cs}$ source on the MCP detector. 
The MCP detector is also tested with a higher-energy ${ }^{124} \mathrm{Sb}$ gamma source that emits a $1.3 \mathrm{MeV}$ gamma, twice the energy of ${ }^{137} \mathrm{Cs}$ source. The exposure rate was $0.33 \mathrm{R} / \mathrm{hr}$ at $1 \mathrm{~m}$ for the ${ }^{124} \mathrm{Sb}$ source. Figure 3.9 shows the effect of the ${ }^{124} \mathrm{Sb}$ source at $1 \mathrm{~m}$ from the detector. Figure 3.9a shows a raw digital radiograph of the cadmium strip with the ${ }^{124} \mathrm{Sb}$ source near $(1 \mathrm{~m})$ the detector, and Figure $3.9 \mathrm{~b}$ shows the same image after flat-field correction. The ${ }^{124} \mathrm{Sb}$ source seems to have only a minor effect on the detector, and flatfield correction provides adequate removal of the gamma signal. The detector also tested with a mixed $357 \mathrm{mCi}{ }^{124} \mathrm{Sb}, 4.6 \mathrm{mCi}{ }^{122} \mathrm{Sb}$ source, yielding $0.39 \mathrm{R} / \mathrm{hr}$ at $1 \mathrm{~m}$. This source, placed at a distance of $5 \mathrm{~cm}$ from the detector, producing the images shown in Figure 3.10.

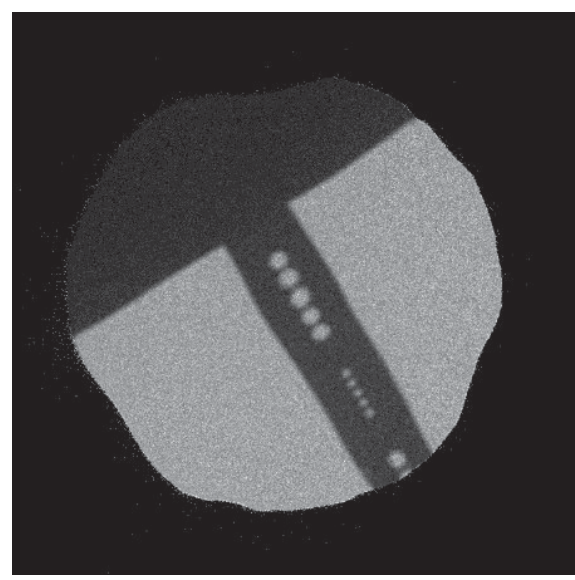

a) Uncorrected radiograph.

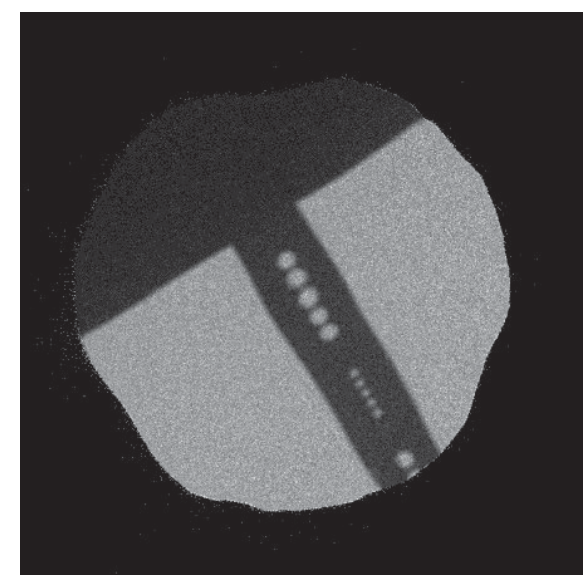

b) Flat-field corrected radiograph.

Figure 3.9. Digital radiographs of a cadmium strip taken with a ${ }^{124} \mathrm{Sb}, 0.33 \mathrm{R} / \mathrm{hr}$ source near the detector.

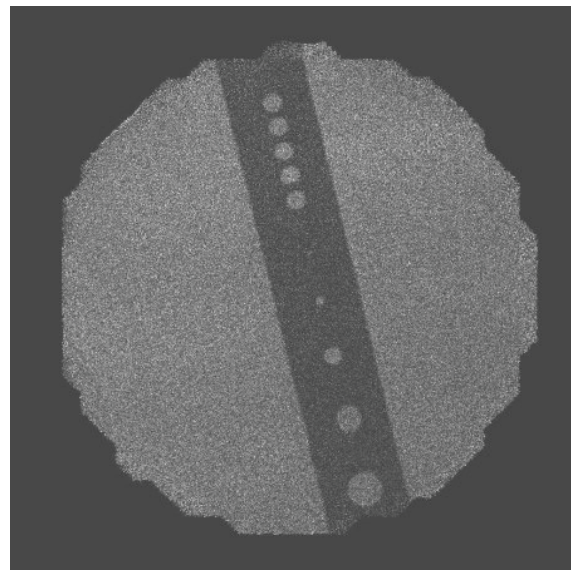

a) Uncorrected radiograph.

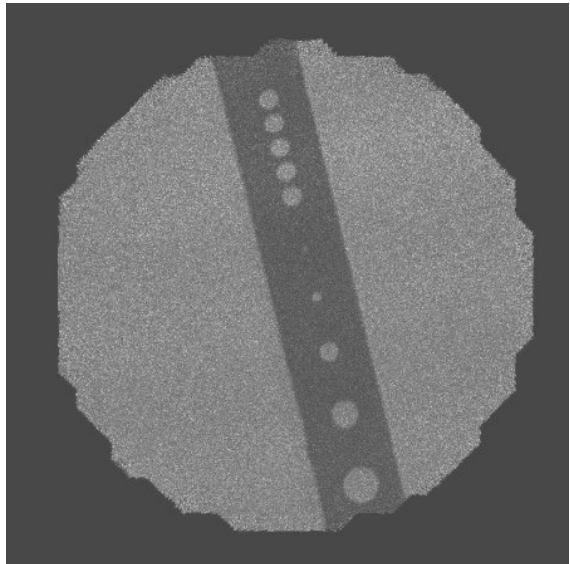

b) Flat-field corrected radiograph.

Figure 3.10. Digital radiographs of a cadmium strip taken with a mixed $357 \mathrm{mCi}{ }^{124} \mathrm{Sb}, 4.6 \mathrm{mCi}{ }^{122} \mathrm{Sb}$ source $5 \mathrm{~cm}$ the detector.

While there is a noticeable response to the gamma source (increased noise), Figure 3.10 suggests that flatfield correction adequately removes the signal from the gamma sources. If gamma intensity does become a problem, which is likely the case for used nuclear fuel emitting $10^{3}-10^{6} \mathrm{R} / \mathrm{hr}$, one possibility would be to add a bismuth gamma filter in front of the detector, which strongly attenuated gamma radiation but has a low absorption and scattering neutron cross-sections. 


\subsection{Determining the Radiography Facility Category According to ASTM Standards}

The MCP detector is tested at the Neutron Radiography Facility (NRF) at Oregon State University (OSU), which has beam port that tangentially-orientated to the reactor core. Neutrons can easily penetrate through the most widely used industrial materials (e.g. steel, lead, and aluminum) but are strongly absorbed by other materials (e.g. hydrogenous materials, boron, gadolinium, cadmium). Thermal neutrons typically provide higher contrast in general compared to higher-energy neutrons, which is the reason thermal neutron are typically used for neutron radiography. The tangential orientation of the NRF neutron beam produces a predominately thermal neutron beam and minimizes the gamma radiation content in the beam. The neutron beam is an ASTM E545 Category-I neutron radiography facility with a thermal neutron flux of $4.6 \times 10^{5} \pm 5.0 \times 10^{4} \mathrm{n} / \mathrm{cm}^{2}$-s. The detector is positioned such that the L/D ratio of the beam is 100 .

The quality of a radiography facility is determined by an ASTM standard ${ }^{10}$, and allows users a means to compare different neutron radiography facilities. There are two image quality indicators (IQI) used to determine the ASTM quality category of a radiography facility, the beam purity indicator (BPI) and the sensitivity indicator (SI). ${ }^{10}$

The SI emulates defects of varying size, with gaps between and holes beneath different thicknesses of acrylic resin. The SI is an aluminum u-channel filled with alternating strips of methylmethacrylate and aluminum, which are milled into steps (Figure 3.11). The aluminum strips (T-Z) simulate gaps and range from $0.254 \mathrm{~mm}\left(0.010 \mathrm{in}\right.$.) to $0.00127 \mathrm{~cm}\left(0.0005\right.$ in.) thick. ${ }^{11}$ There are four methylmethacrylate shims beneath the thicker methylmethacrylate steps, and holes in these shims (1-12) simulate defects and range from $0.508 \mathrm{~mm}\left(0.020 \mathrm{in}\right.$.) to $0.0127 \mathrm{~cm}(0.005 \mathrm{in}$.) in diameter (Figure 3.11$) .{ }^{11}$ A radiograph of the SI is taken and the quantitative determination of the radiographic quality is determined by visually inspecting the SI for the number of visible simulated defects (holes and gaps). ${ }^{10}$

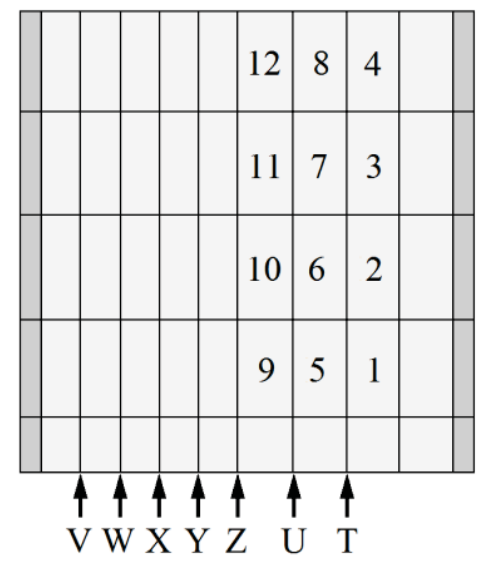

a) Top-view schematic of the sensitivity indicator.

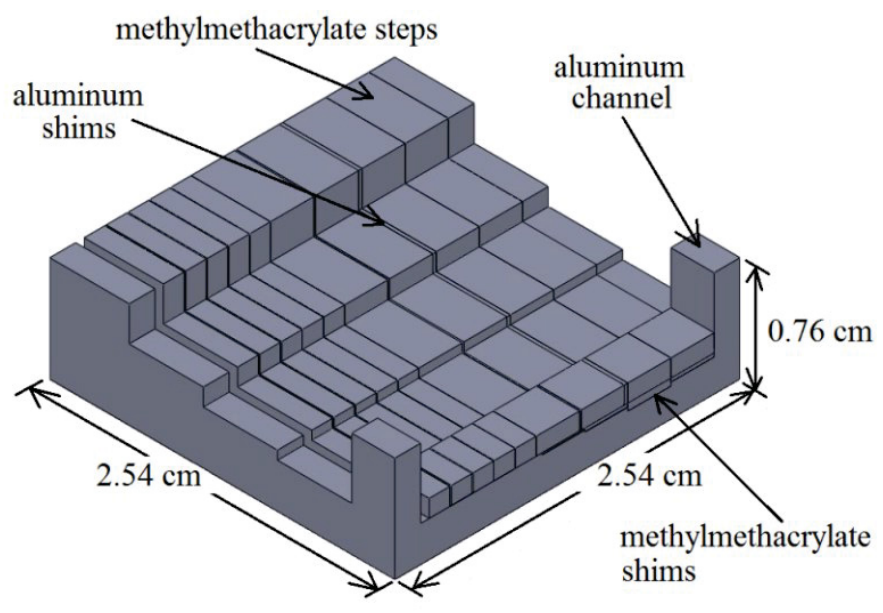

b) Perspective view of the sensitivity indicator.

Figure 3.11. Schematic of the sensitivity indicator.

The BPI is constructed of a block of polytetrafluoroethylene with a hole cut out of its center, and contains two boron nitride discs, two lead discs and two cadmium wires (Figure 3.12). ${ }^{12}$ A combination of each material is mounted on each side of the block. Optical density measurements listed in Table 3.1 are taken from the radiograph of the BPI in locations indicated by the variables shown in Figure 3.12a. 


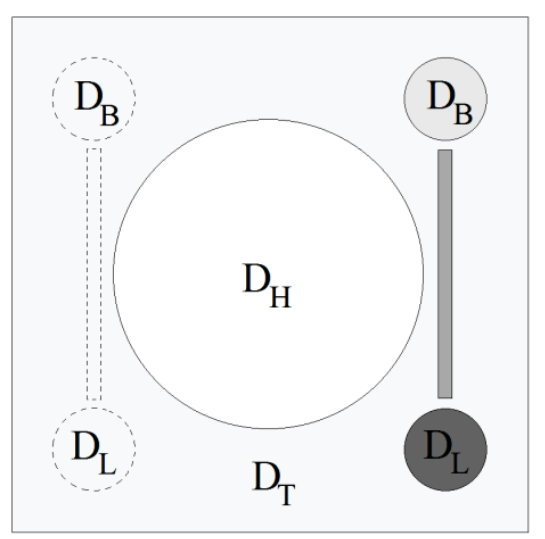

a) Top-view of the beam purity indicator.

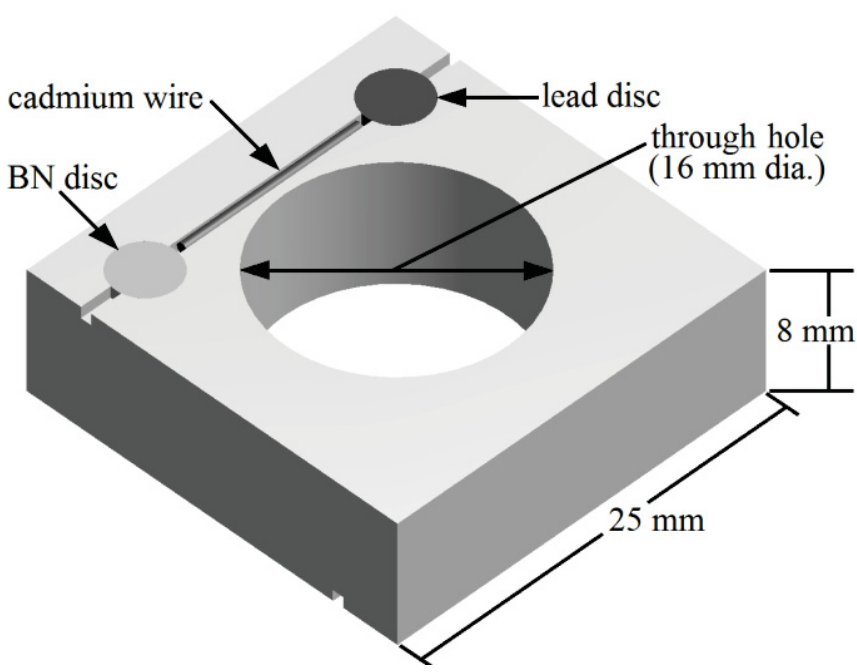

b) Perspective view of the beam purity indicator.

Figure 3.12. Schematic of the beam purity indicator.

Table 3.1. Variable definitions for calculation of ASTM radiography facility parameters.

\begin{tabular}{cl}
\hline Variable & Description \\
\hline DB & Film densities measured through the images of the boron nitride disks \\
DL & Film densities measured through the images of the lead disks \\
DH & Film density measured at the center of the hole in the BPI \\
DT & Film density measured through the image of the PTFE \\
$\Delta$ DL & Difference between the DL values \\
$\Delta$ DB & Difference between the two DB values \\
\hline
\end{tabular}

Table 3.2 lists the beam parameters that determine a facility's ASTM category ratings. ${ }^{10}$ A category-I facility is the top rating. Measurements taken by visual inspection of a radiograph of the SI and optical density measurements from the radiograph of the BPI provide parameters necessary for determining the category of the radiography facility. The parameters in the table represent different measures of beam quality, where $N C$ is the thermal neutron content, $H$ is the smallest hole visible in the SI, $G$ is the smallest gap visible in the SI, $S$ is the scattered neutron content, $\gamma$ is the gamma content, and $P$ is the pair production content. The parameters in Table 3.2 are calculated using the following equations from the ASTM standard. ${ }^{10}$

Table 3.2. Parameters to determine the ASTM radiography facility category.

\begin{tabular}{ccccccc}
\hline Category & NC & H & G & S & $\gamma$ & P \\
\hline I & 65 & 6 & 6 & 5 & 3 & 3 \\
II & 60 & 6 & 6 & 6 & 4 & 4 \\
III & 55 & 5 & 5 & 7 & 5 & 5 \\
IV & 50 & 4 & 5 & 8 & 6 & 6 \\
V & 45 & 3 & 5 & 9 & 7 & 7 \\
\hline
\end{tabular}




$$
\begin{gathered}
N C=\frac{D B-(\text { higher } D B+\Delta D L)}{D B} \times 100 \\
S=\frac{\Delta D B}{D B} \times 100 \\
\gamma=\frac{D T-\text { lower } D L}{D H} \times 100 \\
P=\frac{\Delta D L}{D H} \times 100
\end{gathered}
$$

The value of $H$ is the smallest consecutive visible hole (see Figure 3.11a), and the numerical value of $G$ corresponds to the smallest visible gap according to Table 3.3. The assessment of both $H$ and $G$ are assessed by visual inspection of the radiograph of the SI.

\begin{tabular}{cc} 
Table 3.3: ASTM Standard Value "G" \\
\hline $\begin{array}{c}\text { Gap Label } \\
\text { (see Figure 3.11a) }\end{array}$ & Value of $G$ \\
\hline T & 1 \\
U & 2 \\
V & 3 \\
W & 4 \\
X & 5 \\
Y & 6 \\
Z & 7
\end{tabular}

In order to collect these radiographs, the BPI and SI should be placed next to one another in the same plane as the detector face and in the same image. However, due to size constraints from the detector area and the parallel beam, radiographs of the SI and BPI are acquired separately. The BPI, images shown in Figure 3.13, are acquired over one minute and processed according to the ASTM standard. ${ }^{10}$ Figure 3.14 shows radiographs of the SI acquired and processed with a flat-field and contrast enhancement corrections.

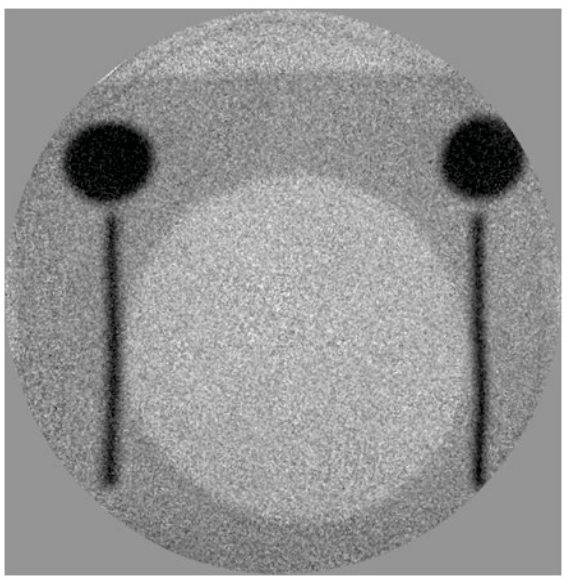

a) View of BPI front face.

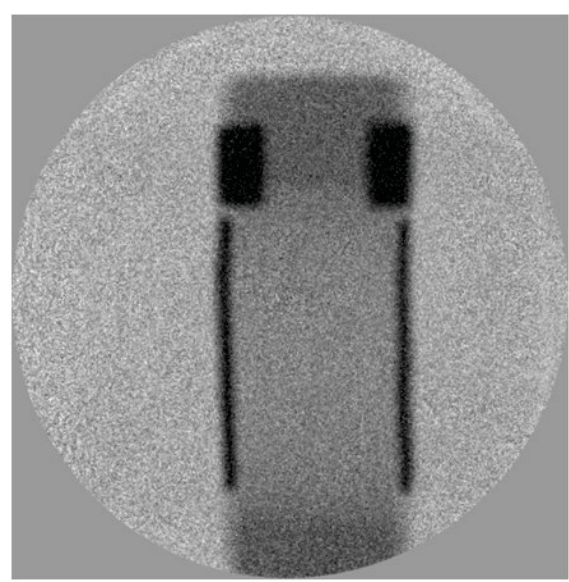

b) BPI orthogonal view.

Figure 3.13. Digital radiographs showing orthogonal views of the BPI. 


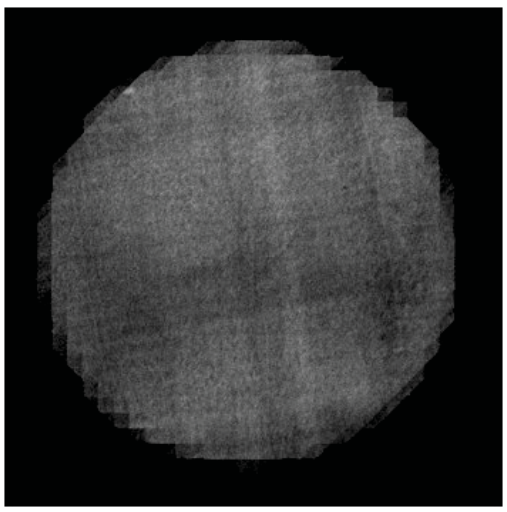

a) Flat-field image.

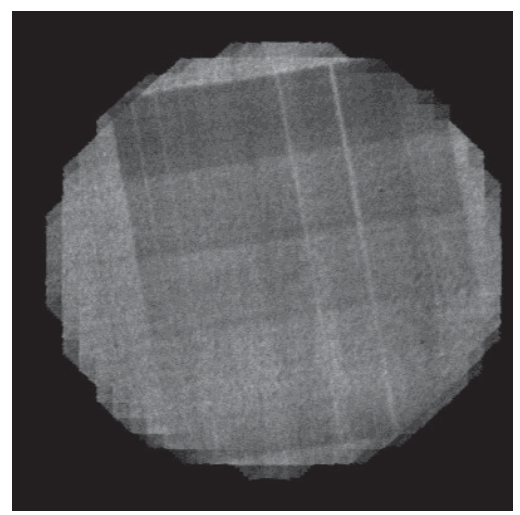

b) Uncorrected radiograph of the SI.

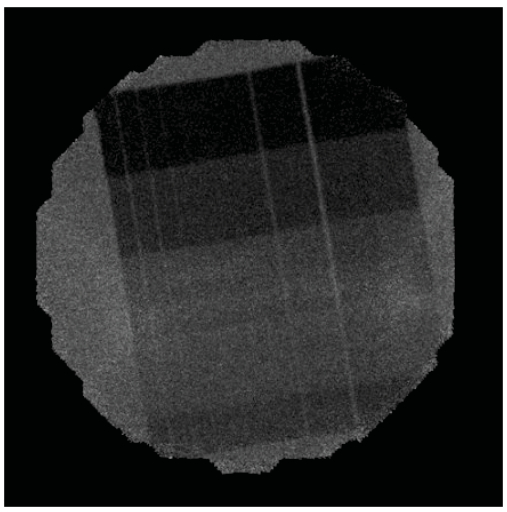

c) Corrected radiograph of the SI.

Figure 3.14. Digital radiographs of the SI showing effect of flat-field correction.

The acquisition time for the images of the SI were longer than for the BPI due to the lower contrast of the materials in the SI compared to the materials of the BPI. The longer exposure times decrease the effectiveness of the flat-field correction because of the dynamic nature of the background inherent to the detector system. Through extensive adjustment of the threshold and varying the bias voltage across the detector, a higher-contrast radiograph of the SI is obtained and is shown in Figure 3.15.

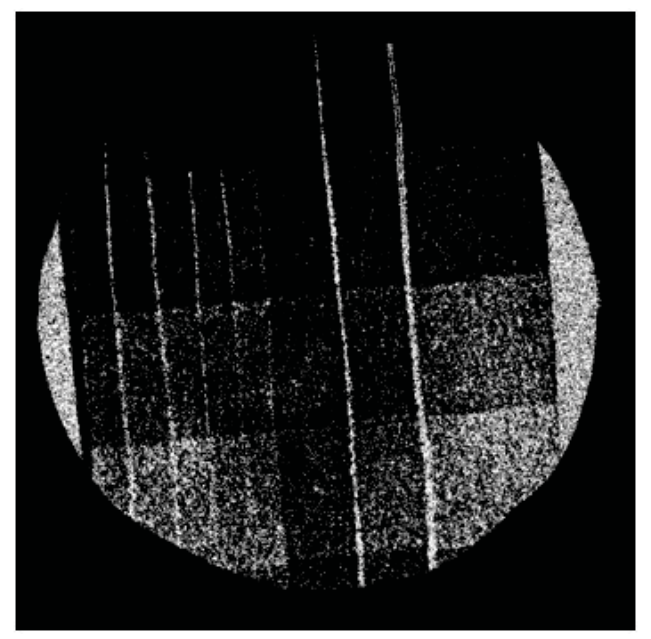

Figure 3.15. Digital radiograph of the SI after significant adjustment of the threshold settings.

Using these radiographs the standards were analyzed to calculate the parameters discussed. These values are compared to Table 3.2 to determine the facility category, and the results are listed in Table 3.4. The results suggest that the detector falls into a class II-III radiography system. From the minimum shim thickness visible, the spatial resolution of the detector is estimated to be between $25-50 \mu \mathrm{m}$ after image processing.

Table 3.4. ASTM Radiography facility category parameters.

\begin{tabular}{lllllll}
\hline Parameter & $N C$ & $H$ & $G$ & $S$ & $\gamma$ & $P$ \\
\hline Measured Value & 65 & 6 & 5 & 7 & 4 & 3 \\
ASTM Category & I & II & III & III & II & I \\
\hline
\end{tabular}




\subsection{Tomographic Reconstruction of a Beam Purity Indicator}

Neutrons passing through a medium are attenuated according to the total macroscopic cross-section of the medium. Tomographic reconstruction processes $2 \mathrm{D}$ radiographs, or projections, to produce a 3D reconstruction of the total macroscopic cross-section of the internal structure of a specimen.

Ideally, radiographs should be taken over $180^{\circ}$ around the object. ${ }^{13}$ The set of radiographs are processed using a filtered backprojection algorithm, which assumes that each projection represents an independent measurement of the object by using a Dirac Delta function. For each axial location, the 1D array of pixel values of each radiograph are combined into a 2D sinogram. For 2D radiographs, multiple sinograms are created, one for each line of projected data.

Recall the BPI standard from the previous section. Radiographs are acquired of the BPI at multiple angles covering $180^{\circ}$ around the BPI. Using the filtered backprojection (FBP) algorithm, each level can be represented with its own sinogram, as illustrated in Figure 3.16.

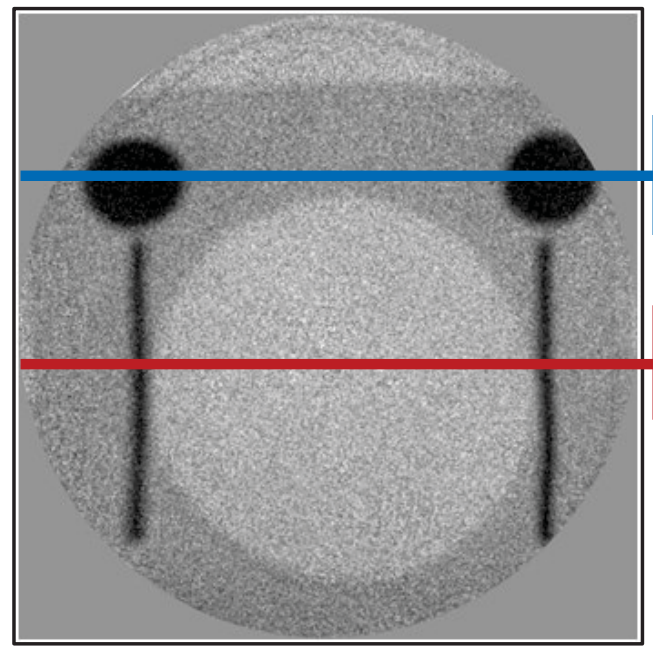

a) Radiograph of the BPI.

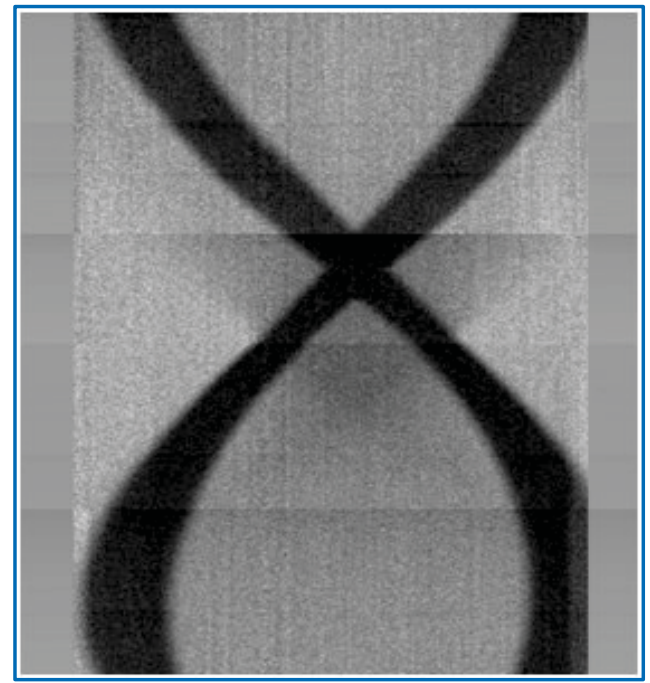

c) Sinogram of axial location with boron nitride discs.

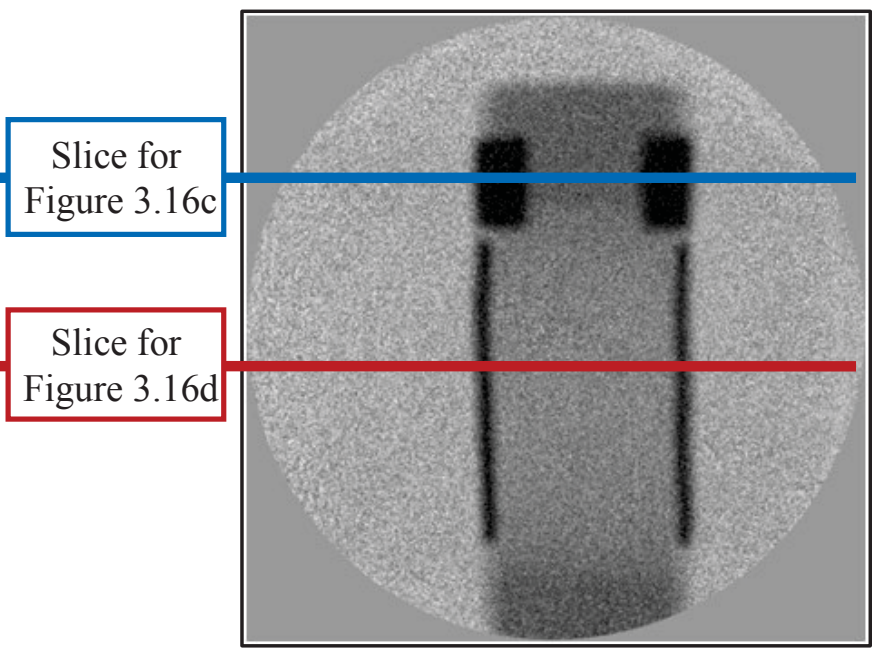

b) Orthogonal radiograph of the BPI.

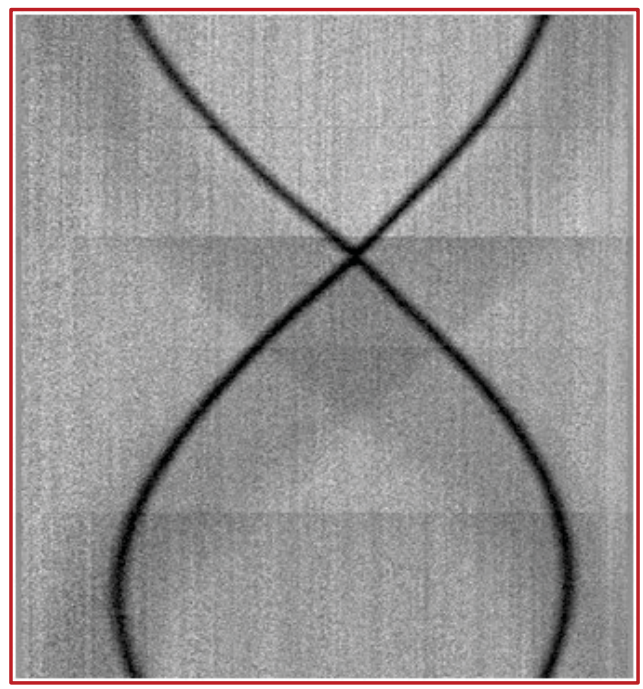

d) Sinogram for axial location with cadmium wires.

Figure 3.16. Orthogonal radiographs of the BPI and respective sinograms. 
The sinogram in Figure 3.16c corresponds to an axial slice passing through the boron nitride disc (blue line in Figure 3.16a,b), which is constructed in $1^{\circ}$ increments. Likewise, the sinogram in Figure 3.16d corresponds to an axial slice passing through the cadmium wire (red line in Figure 3.16a,b). These sinograms are processed in a computer program using FBP to produce 2D reconstructed slices of the BPI, which are shown in Figure 3.17.

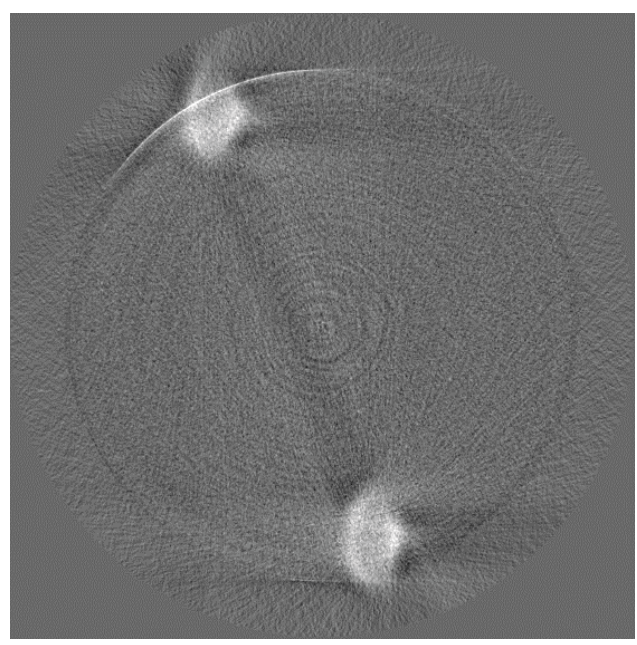

a) Axial location with boron nitride discs.

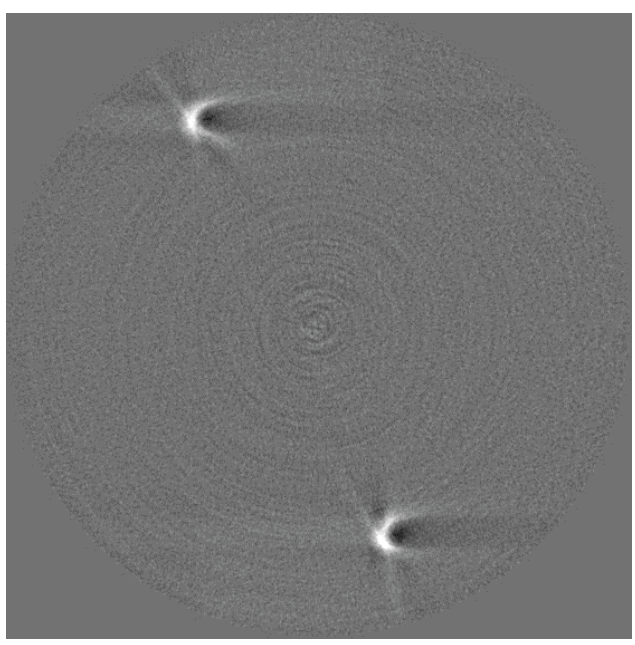

b) Axial location with the cadmium wires.

Figure 3.17. Two-dimensional slices of a tomographic reconstruction of a BPI.

Figure 3.18 shows a 3D representation of the tomographic reconstruction of the BPI. Dimensional measurements of the internal structure of a specimen can be extracted from 3D tomographic reconstructions (such as the reconstruction in Figure 3.18) that may be unavailable with any other nondestructive technique.
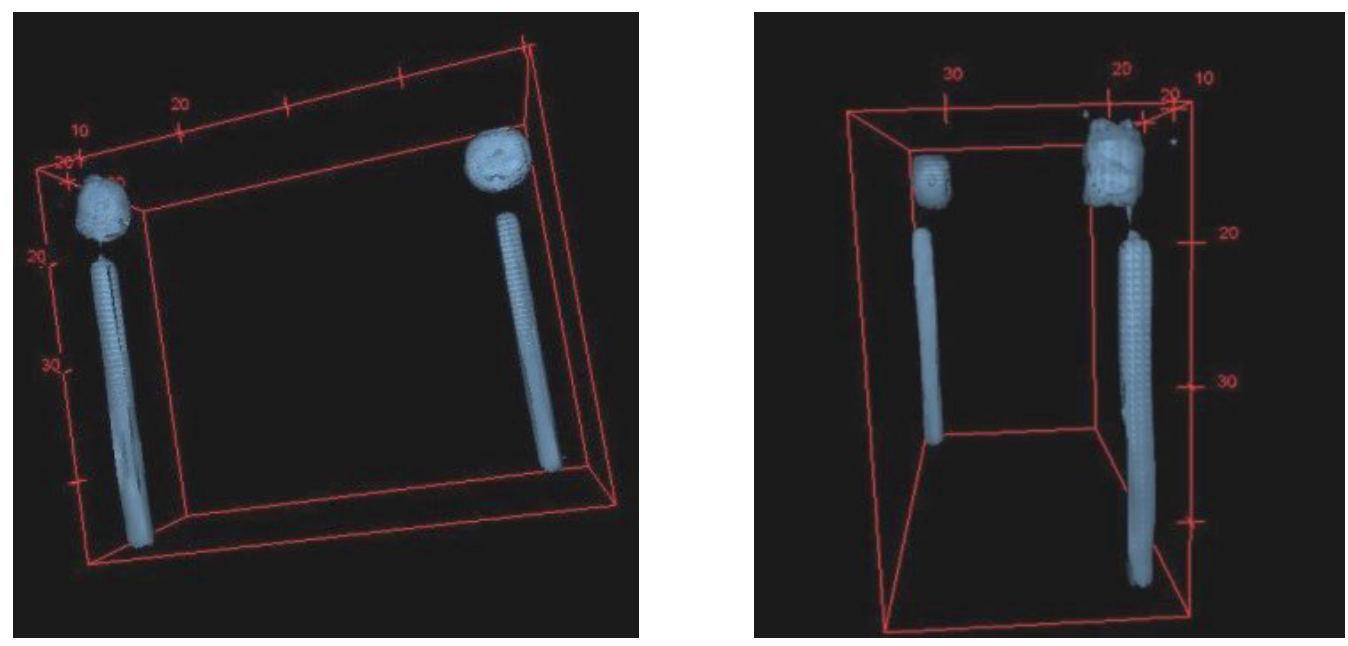

Figure 3.18. Three-dimensional representation of the tomographic reconstruction of the BPI. 


\subsection{Applications and The AFIP-7 Fuel Element}

This same tomographic reconstruction technique can be applied to more complicated objects, such as an AFIP-7 fuel element. The AFIP-7 fuel experiment is a long, plate-type fuel element made up of four seperate fuel plates held together in a bowed congfiguration to allow for expansion. An aluminumn mockup of the AFIP-7 element is tested at OSU for tomographic reconstruction. Aluminum has a very low neutron cross-section, and gadolinium oxide $\left(\mathrm{Gd}_{2} \mathrm{O}_{3}\right)$ is painted along the surface to provide contrast. Figure 3.19 shows orthogonal radiographs showing the front face and side of the element.

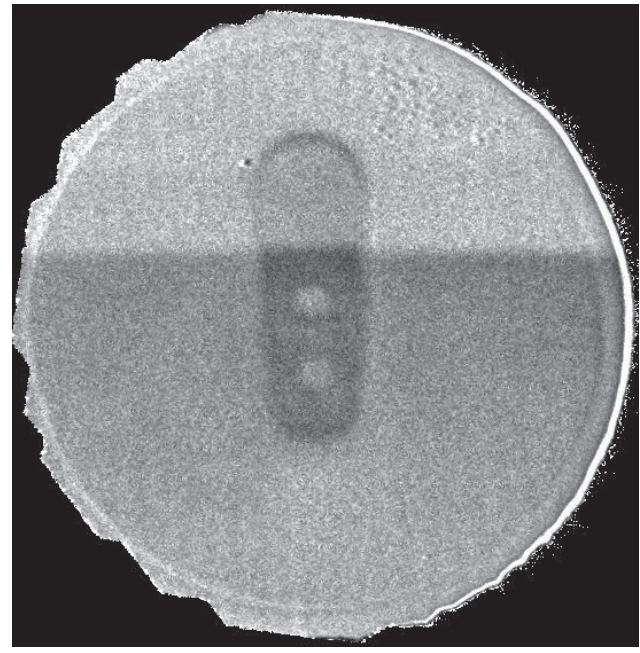

a) Front-face of the AFIP-7 mock-up.

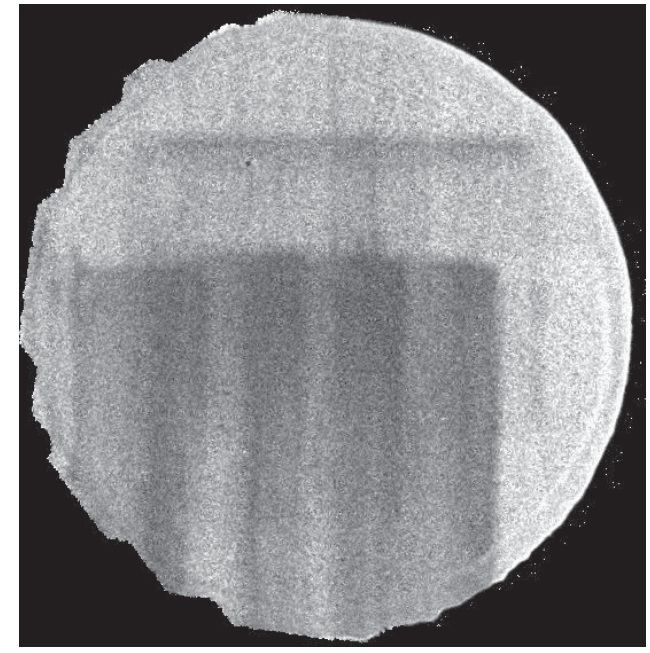

b) Side-view of the AFIP-7 mock-up.

Figure 3.19. Orthogonal radiographs of AFIP-7 mock-up element.

Figure 3.20 shows two sinograms created from radiographs acquired at multiple angles over $180^{\circ}$. The complexity of the sinograms increases significantly with specimen complexity, which can be seen by comparing the sinograms of the relatively simple BPI (Figure 3.16) and relatively complex AFIP-7 mockup (Figure 3.20).
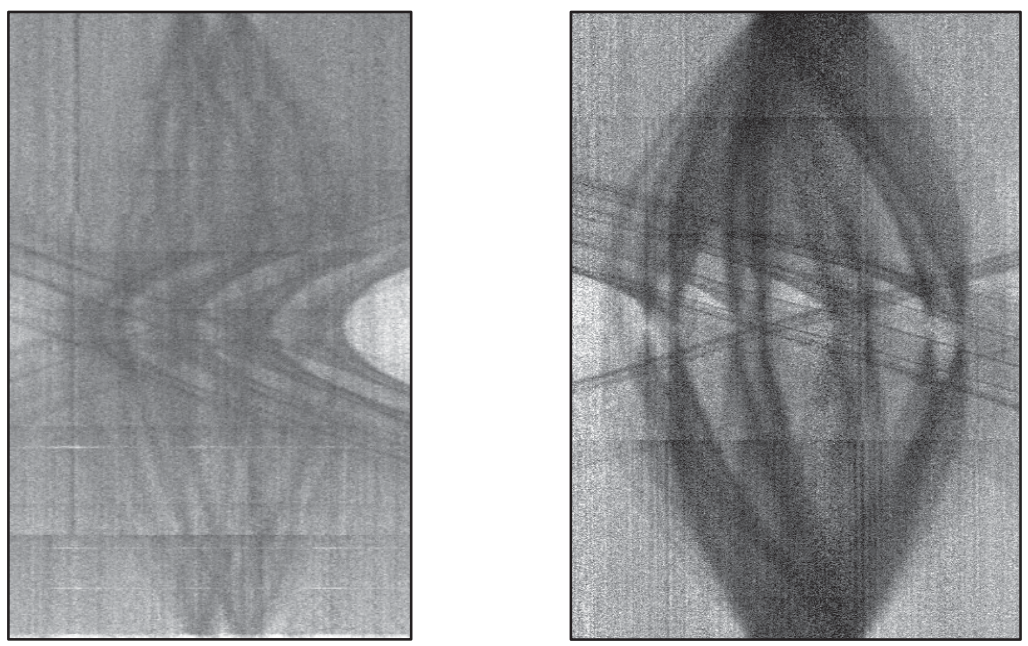

Figure 3.20. Sinograms of the AFIP-7 mock-up. 
The sinograms are processed into a 3D tomographic reconstruction. Figure 3.21 shows a schematic of the AFIP-7 element and the reconstruction of the element looking down the coolant channels. To enhance the contrast of the reconstruction, multiple tomographic slices are superimposed onto one another to produce the 2D rendering shown in Figure 3.21.

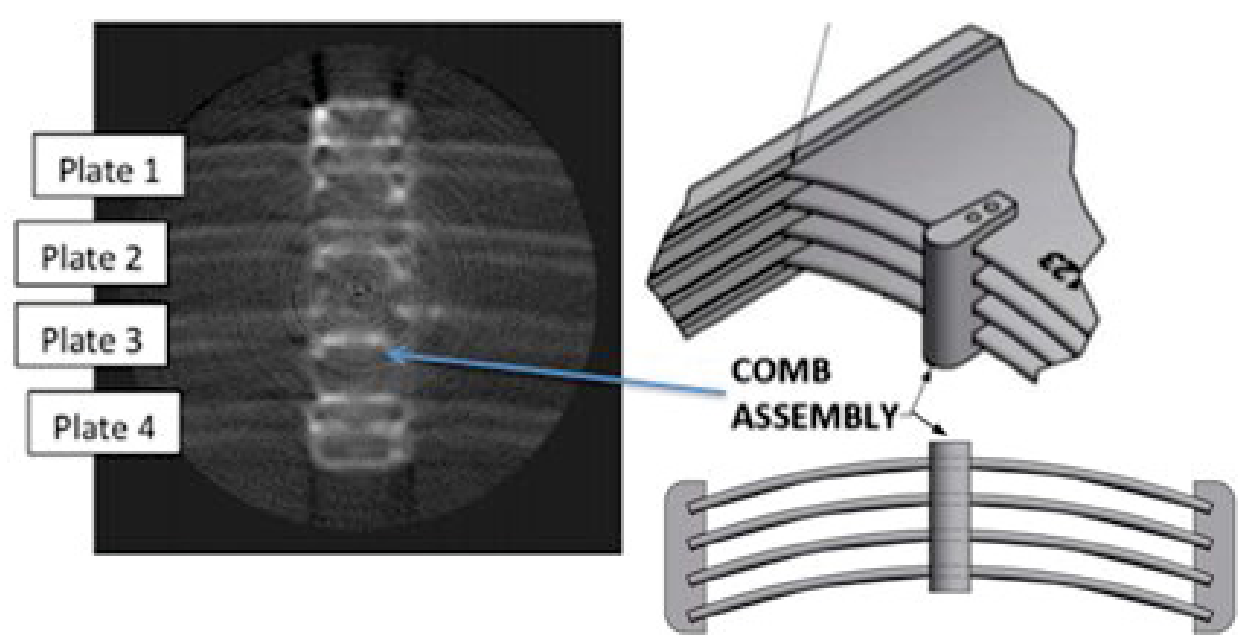

Figure 3.21. Tomographic slice of the simulated AFIP-7 fuel element. 


\subsection{Summary of the MCP System}

The MCP detector developed by NOVA Scientific uses boron-doped glass MCPs to perform digital neutron radiography. Neutrons interact with the ${ }^{10} \mathrm{~B}$ in the MCP by a ${ }^{10} \mathrm{~B}(\mathrm{n}, \alpha){ }^{7} \mathrm{Li}$ reaction that converts neutrons into charged particles. The $\alpha$ and ${ }^{7} \mathrm{Li}$ products, moving $180^{\circ}$ apart, pass from the channel wall surfaces into an open MCP channel, generating free electrons. A bias voltage of $\sim 1-2 \mathrm{kV}$ causes a strong electron avalanche down the micro-channels, which are electronically collected as a pulse in the detector and reconstructed into a $2 \mathrm{D}$ image.

Geometric distortions caused by electronics issues prevent extraction of accurate dimensional measurements from unprocessed radiographs produced by this detector. A radiograph of a pinhole mask and other calibration specimens are used to create a correction algorithm that removes the geometric distortion, making it possible to make accurate dimensional measurements. The maximum spatial resolution is approximately $25 \mu \mathrm{m}$. However, the contrast of the material plays a large role in the final resolution and must be taken into account as materials vary.

The relatively high speed of image acquisition reduces the time to acquire a set of radiographic projections for neutron computed tomography compared to some other techniques for neutron radiography. While the small active area of the detector makes its use for neutron radiography of larger specimens impractical, for small specimens that fit in the $\sim 25 \mathrm{~mm}$ active area, neutron radiography with MCP detectors is more practical and much faster than transfer neutron radiography techniques.

While typical computed tomography procedures require that the images are collected simultaneously and normalized with the same set of background images, this is not feasible with this system because of the dynamic background intrinsic to the detector. The dynamic background is managed by periodic acquisition of flat-field images for image correction. This correction works well for objects that are in the active area of the detector, but the periodic acquisition of flat-field images becomes prohibitively burdensome for objects larger than the active area of the detector. Collecting fewer flat-field correction images produces artificial variations in image intensity, which can drastically affect the quality of a resulting tomographic reconstruction and make regions of the specimen appear more/less thick/dense than in reality.

The MCP detector in this study has the spatial resolution and image acquisition time required to produce neutron radiographs for tomographic reconstruction. The inconsistency of the radiographs caused by the dynamic background requires the acquisition of twice the number of radiographs in order to produce a set of radiographs suitable for computed tomography.

Future work at the INL consists of using the MCP detector for radiography of spent nuclear fuel to test the gamma sensitivity at significantly higher gamma dose rates and perform computed tomography on materials with higher neutron attenuation cross-sections (nuclear fuel). These high cross-sections will allow for better contrast, which may significantly reduce the number of radiographs required to produce a quality tomographic reconstruction and would also reduce image acquisition time. 


\section{NEUTRON COMPUTED RADIOGRAPHY}

The NRAD currently performs neutrons radiography by the foil-film transfer method. Film processing is the largest contributor to the time it takes to produce an image, and includes exposure to the activated foils, wet film processing to develop the film, and scanning into a high-resolution digital image. Computed radiography (CR) imaging systems utilize photostimulable storage phosphor (PSP) plates, often called phosphor storage plates or imaging plates (IP), to produce quality digital x-ray radiographs. Neutron computed radiography uses the same foil transfer method for neutron radiography that is already employed at the NRAD (see Section 2), but rather than coupling the activated foils to film, the foils are coupled to IPs. Figure 4.1 describes the process for transfer method neutron CR.

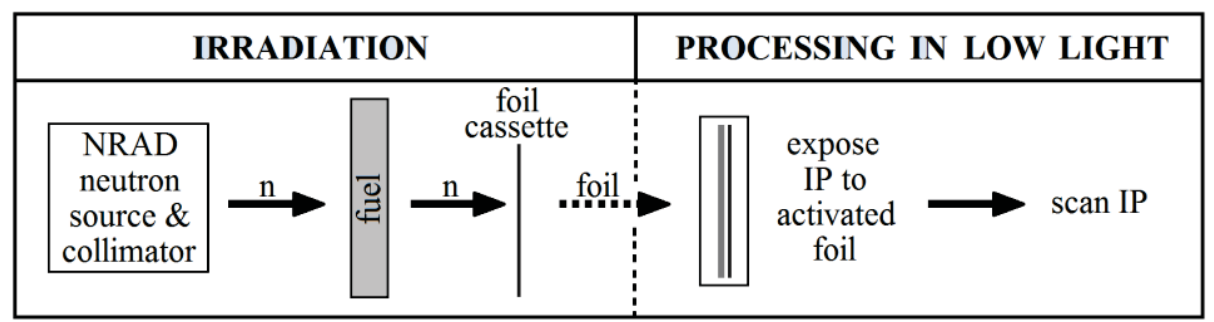

Figure 4.1. Schematic of the neutron computed radiography process.

The foils in the cassette are activated by the same process as before. The activated foils are taken to a low-light room where an IP is coupled to the activated foils, and the activated foils are allowed to decay overnight. The decay radiation from the activated foils stimulates the phosphor layer of the IP in the pattern of the attenuated beam through the specimen, just as with film, thus recording the latent image as a pattern of photo-stimulation on the IP. The operator then places the IP in a scanner, which scans the IP with a red laser to release the stored energy as blue light. A photo-multiplier tube amplifies the blue light pulse into a usable signal using a photo-multiplier tube.

The IPs must still be exposed to the activated foils like film. Neutron CR does not require wet film development, and thus reduces processing time compared to film. The CR system is capable of producing a digital image from the exposed IP minutes after exposure, which significantly cuts the time required to produce an image. Acquiring an image with film takes at least two days, whereas it is possible to obtain a digital image in one day using neutron $\mathrm{CR}$.

IPs are more sensitive than film, and thus require less exposure to acquire an image. With neutron CR, the higher sensitivity of IPs correlates to shorter neutron beam exposure time to the foil cassette. This not only saves neutron beam exposure time, cutting the beam time roughly in half, but also reduces the activity of the dysprosium and indium foils, thus further reducing the already low dose received by operators handling the activated foils.

A CR system consists of IPs, a scanner, and a computer system for operating the scanner, displaying the image, and processing the image file. The NRAD owns a ScanX 14 Portable scanner manufactured by AllPro Imaging. A computer runs a software package that allows the user to control the scanner and manipulate the scanner settings. There are different types, manufacturers, grades, etc. of imaging plates that are commercially available, and the same scanner works for all of the PSP plates. The following section discusses PSP IPs in greater detail. 


\subsection{Photostimulable Storage Phosphor Imaging Plates}

Computed radiography is almost exclusively applied to x-ray radiography. In x-ray CR, PSP imaging plates store absorbed x-ray energy in a crystal lattice structure. In neutron CR, the plates are exposed to the decay radiation from activated foils, creating an unobservable latent image stored in the crystal traps. This stored energy is later released and read through photo-stimulated luminescence using a digital scanner by stimulating the lattice and inducing light emission. A red laser stimulates small areas of the IP, causing the lattice to release the stored energy as blue light. The ScanX image plate reader rasters a red laser over the IP, and the emitted blue light signal is read at each location and is used by the software to generate a digital image.

Unlike film, IPs are reusable. The red laser used to scan the IP slightly reduces the intensity of the image stored on the IP, but the IP can be rescanned if desired. The phosphor plate still contains a residual image after scanning, which must be removed by exposing the screen to bright, preferably red light for a period of time (typically around two minutes). After the IP is erased, it is ready for reuse for another exposure. The operator should avoid using sunlight to erase the IP, since sunlight contains UV light that can deeply stimulate the phosphor and leave artifacts in the image. The ScanX scanner includes an in-line erase function that automatically erases the IP while scanning. Each IP should be erased within 24 hours prior to use (i.e. exposure and scanning) since background radiation will add noise to the IP.

Because IPs are sensitive to visible light, leaving an exposed but un-scanned IP in light will cause loss of image quality. Thus, removal of an exposed IP from the cassette and insertion into the scanner should be administered in low-light conditions. The operator should not process the IPs in dark-room red safe-light conditions, since red safe-lights erase the image similarly to white light.

There are a number of manufacturers of imaging plates capable of producing quality images, including Kodak (which is manufactured by Carestream), Fuji, GE, AGFA, FlexCR, and others. The two largest manufacturers of IPs are Kodak and Fuji. The most notable difference between similar-grade IPs made by Kodak and Fuji is that Kodak IPs have a more robust protective coating over the PSP crystal layer A thicker protective coating reduces damage to the IP and extends operational lifetime, but also results in a slight decrease in effective spatial resolution. Fuji IPs will have slightly better spatial resolution capabilities than a similar-grade of Kodak IP, but the protective coating is lacking compared to Kodak.

Kodak and Fuji both offer general purpose (GP) and high-resolution (HR) imaging plates. The GP plates provide adequate spatial resolution for general purpose applications. If higher spatial resolution is required, the HR plates may be desirable; the spatial resolution of HR plates is limited to around $50 \mu \mathrm{m}$. While the HR plates offer improved spatial resolution, they require almost twice the exposure dose compared to GP plates. Some manufacturers also offer a very high resolution IP, often a translucent blue plate, that provides higher resolution capabilities than even the HR series; however, these plates often require more than twice the dose of the HR plates. 


\subsection{Spatial Resolution of Transfer Method Neutron Radiography Techniques}

Like film, the spatial resolution of an image produced by transfer method neutron CR is affected by many factors. First, factors associated with the NRAD facility up to the point of producing activated dysprosium and indium foils should be the same for both film and neutron CR, with the exception of less neutron beam exposure required for neutron CR. Other factors that affect spatial resolution are unique to film and IPs. The following subsection briefly discusses the factors that affect spatial resolution for transfer method neutron radiography with both film and IPs.

\subsubsection{Factors Affecting Spatial Resolution for Transfer Method Neutron Radiography Techniques}

Some of the factors that affect spatial resolution are the same for transfer method neutron radiography with both film and CR. While these factors are the same for both film and IPs, their effects on spatial resolution warrant discussion because some of these factors can be manipulated to improve spatial resolution. Some of these factors include the L/D ratio of the neutron beam, activation foil thickness and process quality control.

The L/D ratio of a neutron beam describes the level of collimation of the neutron beam. Increasing the $\mathrm{L} / \mathrm{D}$ ratio produces a more collimated the neutron beam but decreases the beam intensity, requiring increased beam exposure times to achieve the same foil activities. The $\mathrm{L} / \mathrm{D}$ ratio can be adjusted by increasing the aperture diameter (D), and the ERS beam has three L/D settings $(50,125,300)$, and typically operates at L/D of 125 . The reduced exposure required for neutron $\mathrm{CR}$ may offset the lower beam intensity associated with increasing the L/D to 300 .

The thickness of the activation foil also affects spatial resolution due to geometric factors. Scattering of the neutron beam in the specimen and other structural materials can contribute to the activation of the foils, but are a source of noise rather than the desired signal of unattenuated neutrons. This becomes more significant for thick specimens with high scatter cross-sections. The effects of scattered neutrons can be reduced by adding a thin neutron filter between the specimen and the activation foils.

The unattenuated neutrons interact with the transfer foils and may undergo scattering interactions before finally being absorbed by the foil material, which activates the foil. Figure 4.2 shows a qualitative schematic of an impinging neutron beam on a foil. The neutrons scatter out of their original trajectory as they travel through the material, and the resulting volume of interaction resembles a pear shape.

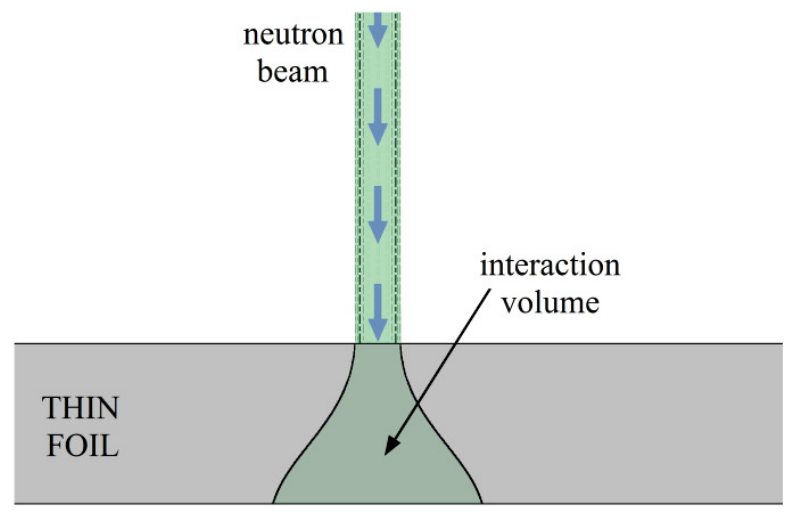

a) Interaction volume with a thin foil.

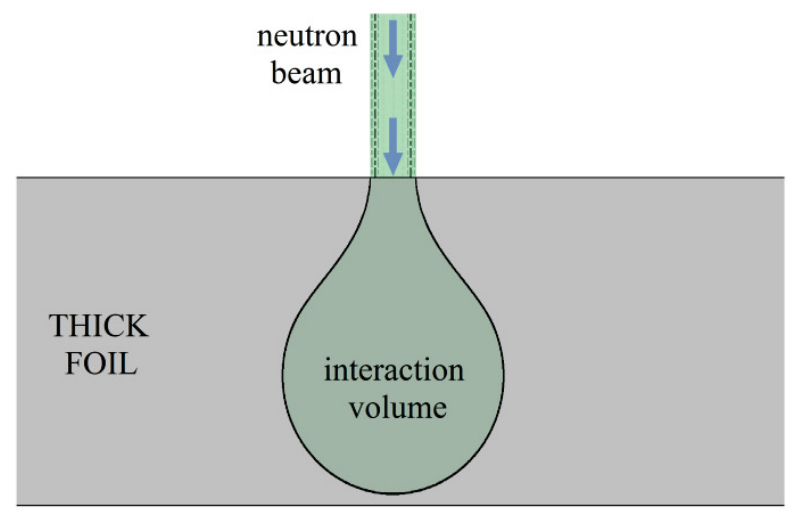

b) Interaction volume with a thick foil.

Figure 4.2. Qualitative schematic of an impinging neutron beam on a foil. 
Ideally, a neutron would activate the foil in the same location it first enters the foil. However, because of scattering in the foil, some neutrons are absorbed in locations far from the location they initially entered the material, which negatively impacts the spatial resolution of the resulting image. Using a thin foil, with a thickness of a fraction of the neutron mean free path of the foil material, reduces the amount of scattered neutrons and the interaction volume. The thicknesses of foils typically used for neutron radiography typically range between $100 \mu \mathrm{m}$ and $200 \mu \mathrm{m}$. This is less than the mean free path of thermal neutron in dysprosium $\left(\bar{x}_{\mathrm{Dy}} \approx 300 \mu \mathrm{m}\right)$ and indium $\left(\bar{x}_{\mathrm{In}} \approx 1.3 \mathrm{~mm}\right)$. The interaction volume and deviation from the original trajectory is also reduced by choosing a material that has a much higher absorption cross-section than its scattering cross-section; such is the case for both dysprosium and indium.

Imperfections in the exposure of the film also negatively impact spatial resolution. Ideally, decay radiation from an activated radionuclide would interact with the film at the same relative location in the plane of the foil/film that the decay took place. However, radionuclides release decay radiation over a $4 \pi$ solid angle. Figure 4.3 is a qualitative schematic that shows the geometric effects of the thickness of an activation foil placed in contact with film. Although radiation is released in all directions, Figure 4.3 shows the radiation trajectory for only a small portion of the solid angle in order to more clearly show the effect of foil thickness on the interaction volume in the film. Neglecting self-shielding within the foil itself, radiation from decayed radionuclides located farther away from the film interact with a larger volume of the film than radiation from decayed radionuclides closer to the film. This leads to degradation of spatial resolution of the resulting image for both IPs and film.

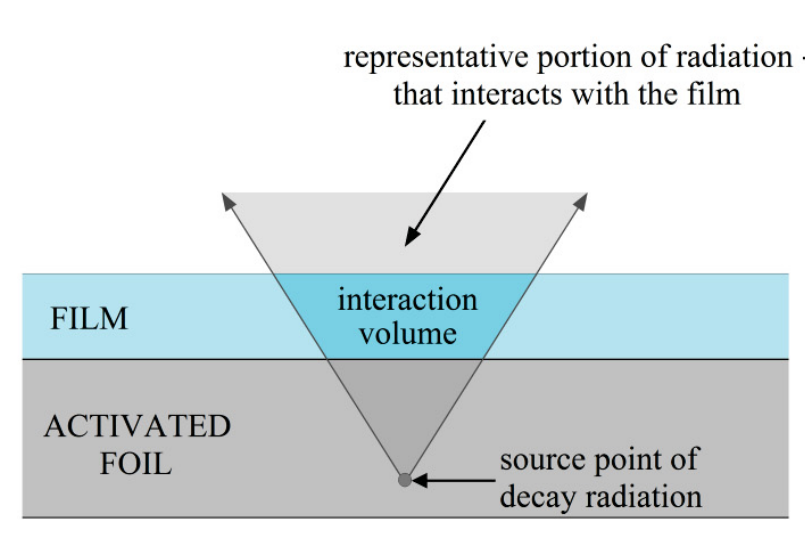

a) Thin activation foil.

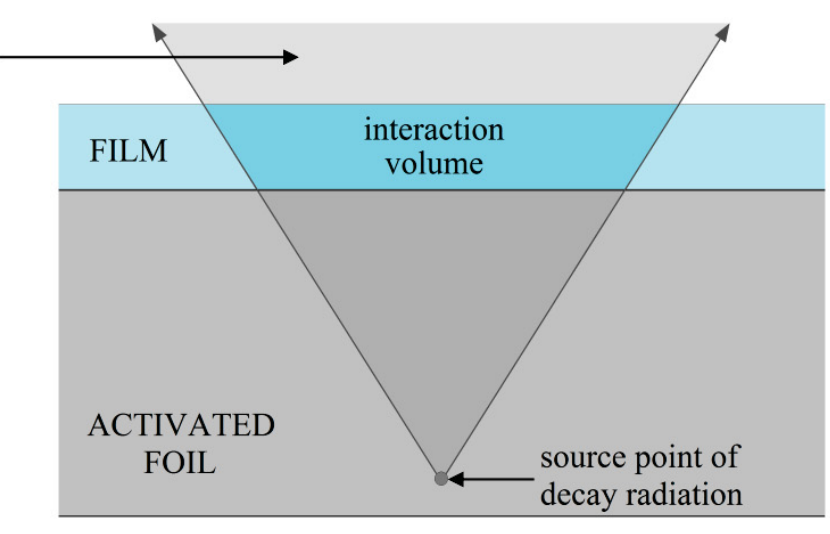

b) Thick activation foil.

Figure 4.3. Spatial effects of the thickness of an activation foil for transfer method neutron radiography.

Reducing the thickness of the activated foil reduces the degradation of spatial resolution by reducing the film interaction volume of the decay radiation from the foil. While this would support using very thin activation foils, the thickness of the foil is also affected by the amount of neutron beam exposure time required to sufficiently activate the foil that it provides the desired exposure to the film. If the foil is too thin, the neutron beam exposure times may become impractically long. Thus, there is a balance between obtaining images with higher spatial resolution and the practical side of operating and processing time.

The same geometric factors described in Figure 4.3 also apply to the thickness of the sensitive layer of the film or imaging plate; a thicker interaction layer has a negative impact on spatial resolution. For this reason, film used for transfer method neutron radiography is typically a single-sided emulsion, and the film is placed into contact with the activated foil with the emulsion-side of the film facing the activated foil. With IPs, a thicker PSP crystal layer negatively impacts spatial resolution. However, thickness of the PSP layer also affects the amount of exposure required to obtain an image, and a thinner PSP layer requires increased exposure 
Other factors that affect spatial resolution pertain to quality control and processing errors. Wet film processing can create artifacts and cause distortion of the image. The wet film processor can also cause damage to the film (e.g. scratch marks from the rollers) or destruction of the film altogether, requiring acquisition of another radiograph. Many of the processing artifacts can be positively identified, but still detract from the quality of the image. Accidental exposure to light has a severe negative impact on the image for both film and IPs, and precautions are taken to reduce the probability of this occurring.

Image plates do not require wet film process, but rather are scanned into a digital image. The scanner settings affect the image quality, and some settings affect the spatial resolution of the resulting image. The PMT voltage, laser rastering speed and laser power affect the amount of blue light signal detected. The scanning resolution (typically ranging between 50-100 $\mu \mathrm{m} /$ pixel) has a significant impact on spatial resolution, and the operator should be familiar with these settings prior to scanning the IP. The IP can be rescanned multiple times with little degradation and should not be erased until a satisfactory image is acquired.

\subsubsection{Comparison of the Spatial Resolution of Foil-Film Transfer Method Neutron Radiography and Neutron Computed Radiography}

In order to quantitatively compare the spatial resolution properties, a modulation transfer function (MTF) is calculated from radiographs taken with both foil-film and neutron CR transfer method. The "resolution" of an imaging system can mean many things, and is often quoted as the pixel size of the resulting digital image. However, effective spatial resolution is a function of contrast; a particular feature becomes less visible with decreasing contrast. The MTF quantifies the contrast as a function of spatial resolution by mathematical processing of an image of an edge specimen that was taken with a particular imaging system. An edge specimen is simply a thin, high-contrast material with a finely-machined edge. If the edge specimen were a theoretical perfect absorber and the imaging system was theoretically perfect, the image of the edge specimen would appear as a step function. However, due to imperfections of the imaging system, the edge appears less defined, and the edge in the resulting image drops off more gradually.

Typically, a user places an appropriate edge specimen in an image for determination of the MTF of the imaging system. However, for radiographs taken without an appropriate edge specimen, a fine edge may still be found in the radiograph that may provide the edge spread function required to calculate the MTF of the system. In these situations, the user could take several edges in the image, calculate the MTF of each edge, and compare each calculated MTF to determine if one of the edges provides an adequate edge spread function. Increasing the contrast of the edge specimen material and increasing the signal to noise ratio produce an MTF with lower noise at high-frequencies.

The image of the edge specimen is processed to produce an edge spread function, which is differentiated to produce a line spread function. The MTF is then the fast Fourier transform of the line spread function. A graph of the MTF has a vertical axis typically labeled as MTF, but can also be considered as contrast (ranging between 0 and 1). The $\mathrm{x}$-axis of the MTF plot is spatial frequency, typically either in line-pairs (lp) per mm or cycles per mm.

Digital images are produced and MTF curves are calculated for each of the following imaging systems to provide a preliminary comparison between film neutron radiography and neutron CR, including: 1) foilfilm transfer method neutron radiography scanned at $600 \mathrm{dpi}, 2)$ transfer method neutron CR scanned at $50 \mu \mathrm{m} /$ pixel, and 3) x-ray CR using the same type of IP and $50 \mu \mathrm{m} /$ pixel scanning resolution. The resulting MTF curves are shown in Figure 4.4. The film neutron radiograph has the best spatial resolution of the three systems (Figure 4.4, solid green line). The spatial resolution of the neutron CR system (Figure 4.4, dashed green line) is less than half that of film. To determine if this significant difference in spatial resolution is truly a product of the CR system, an MTF of the same type of IP is calculated from an x-ray radiograph and scanned with a newer scanner (Figure 4.4, solid blue line). The X-ray CR image has 
slightly lower, but nearly equal spatial resolution to the film neutron radiograph. This indicates that the IPs are capable of producing images with nearly the same spatial resolution as the current foil-film transfer method technique employed at the NRAD.

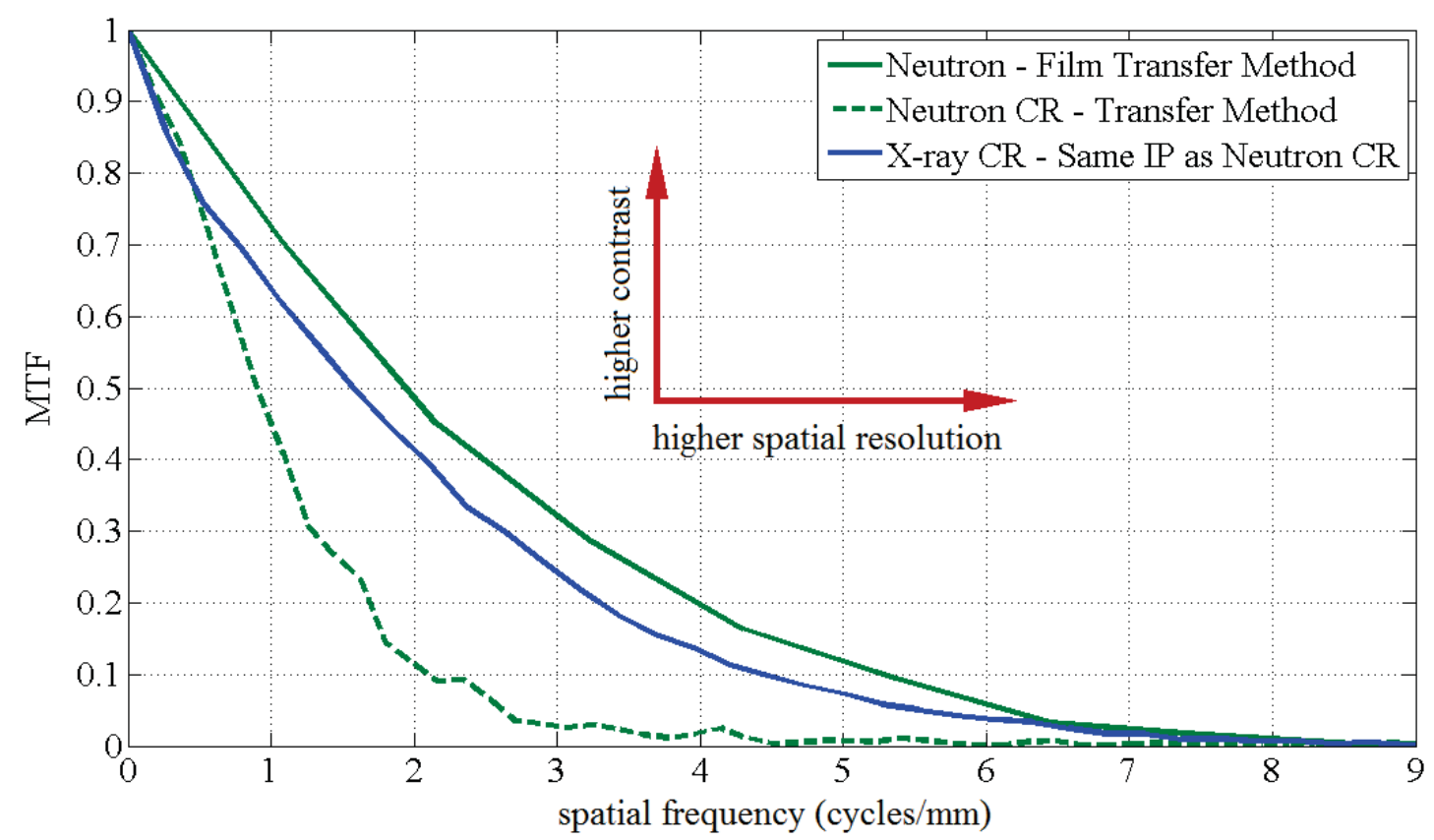

Figure 4.4. MTF of neutron radiography for both film and imaging plates.

These results provide a preliminary comparison between the spatial resolution of foil-film neutron radiography and neutron $\mathrm{CR}$, and demonstrate an opportunity to reduce time and cost of neutron radiography at the INL. Further development and characterization will continue. The MTF data presented are calculated using edge data from different edge specimens. A more accurate comparison of the spatial resolution properties of these systems will be assessed using the same edge specimen for all the systems. Multiple edge specimens are available (e.g. dysprosium, gadolinium, and/or cadmium foils) and MTFs from an image of these specimens will determine which specimen is most appropriate. Also, a new highresolution CR scanner will likely provide neutron CR images with higher spatial resolution than film, and acquisition of such a scanner is being considered.

Advanced neutron radiography detectors, including MCPs and neutron CR, may provide quality images for tomographic reconstruction at significantly reduced cost and time compared to the current foil-film system. Development of these systems makes neutron tomography at the INL an increasingly practical and reasonable possibility, and development of tomographic reconstruction capabilities is also underway. The following section describes the development of neutron computed tomography of nuclear fuel at Missouri S\&T. 


\section{COMPUTED TOMOGRAPHY AT MISSOURI S\&T}

One of the main objectives of this work is to demonstrate the feasibility of tomographic reconstruction of AFIP-7 mock-up using neutron radiographs and extend the idea to digital neutron tomography. This section discusses the proof of principle of 3D neutron tomography using archived images of the TREAT reactor's L07 fuel bundle collected at NRAD.

\subsection{Image Acquisition}

Neutron radiographs of the L07 fuel assembly are produced at the NRAD facility using a nearly parallel beam geometry (L/D ratio is 185$)$. The assembly is rotated in precisely $2.4^{\circ}$ increments, with a radiograph acquired at each angle using the transfer technique at the NRAD. Table 5.1 lists some fuel parameters of the L07 fuel assembly.

\begin{tabular}{lc}
\multicolumn{2}{l}{ Table 5.1. Characteristics of the $\mathrm{L} 07$ fuel assembly. } \\
\hline Parameter & Value \\
\hline Fuel composition & $\mathrm{UO}_{2}+\mathrm{PuO}_{2}$ \\
Fuel diameter $(\mathrm{mm})$ & $4.95-5.00$ \\
Fuel density $\left(\mathrm{g} / \mathrm{cm}^{3}\right)$ & 4.7 \\
Fuel length $(\mathrm{mm})$ & 914 \\
\hline
\end{tabular}

The film is exposed to the decay radiation from the activated dysprosium and indium foils, which causes the film to darken after the film is developed. Increased exposure to decay radiation makes the film darker, indicating higher neutron flux. Areas of the film that are lighter indicate a lower impinging neutron flux.

The optical density (OD) of film is a measure of the light transmittance through the film. The higher the OD, the lower the transmittance and the darker the film. Thus, the OD is inversely correlated with pixel intensity (where a gray value of zero is black). The OD is determined by measuring the ratio of a light source, $\varphi_{\mathrm{o}}(x, y)$, and the transmitted light intensity, $\varphi(x, y)$, over the film $(x, y)$ using the following relationship: ${ }^{14}$

$$
O D(x, y)=-\log _{10}\left(\frac{\varphi(x, y)}{\varphi_{\mathrm{o}}(x, y)}\right)
$$

The OD correlates to the activity of the foil that exposed the film, and the activity of the foil is related to the attenuation of the neutron beam flux by the specimen. The activity of the foil, $\mathrm{A}(t)$, is given by:

where,

$$
A(t)=\Sigma_{a} \phi(x, y)\left(1-e^{-\lambda t_{i r r}}\right)
$$

$$
\phi(x, y)=\phi_{0} e^{-\Sigma_{t} \Delta x}
$$

and where $\Sigma_{a}$ is the macroscopic absorption cross-section of the specimen, $\Sigma_{t}$ is the total macroscopic cross-section of the specimen, $\phi_{\mathrm{o}}$ is the unattenuated neutron beam flux, $\phi(x, y)$ is the neutron beam flux over $x, y$ at the activation foil after passing through the specimen, $\lambda$ is the decay constant of the activated foil, $t_{i r r}$ is the time the foils were exposed to the neutron beam, and $\Delta x$ is the thickness of the specimen. If the composition of the specimen is known, a scanned image of the film radiograph provides the data required to calculate the thickness of the sample by correlating the grayscale intensity map of the image. 
Radiographs of the L07 fuel element are digitized using Genesis NEO S60 film digitizer, which produces a 16-bit image. The digitizer uses cold cathode lamp as light source which can measure optical density up to 4.7 with spatial resolution up to $600 \mathrm{dpi}$. The digitizer has its own user interface module which enables user friendly selection tabs. Table 5.2 lists some technical specifications of the Genesis NEO S60 scanner. The scan rate is $1.5 \mathrm{~ms}$ per line; the total scan time is $55 \mathrm{~s}$ for a $14 \mathrm{in} . \times 17 \mathrm{in}$. film. The size of the neutron radiographs was $25 \mathrm{~cm}(\sim 10$ in. $) \times 45 \mathrm{~cm}(\sim 17$ in.) but since the scanner software has limited option to select film size, the scans were performed at 14 in. $\times 17$ in. and then cropped. Figure 5.1 shows a 3 digitized L07 fuel bundle from three different angles.

Table 5.2. Technical specifications for the Genesis NEO S60 scanner.

\begin{tabular}{ll}
\hline Parameter & Value \\
\hline Maximum resolution & Up to 600 dpi \\
Sensor & High pixel CCD \\
Optical density range & 4.7 maximum \\
Bit depth & 16 -bit \\
Scan rate & 1.5 ms/line, \\
Software & Windows XP, Vista, Windows 7 interface \\
& compatible with OmniVue® \\
\hline
\end{tabular}

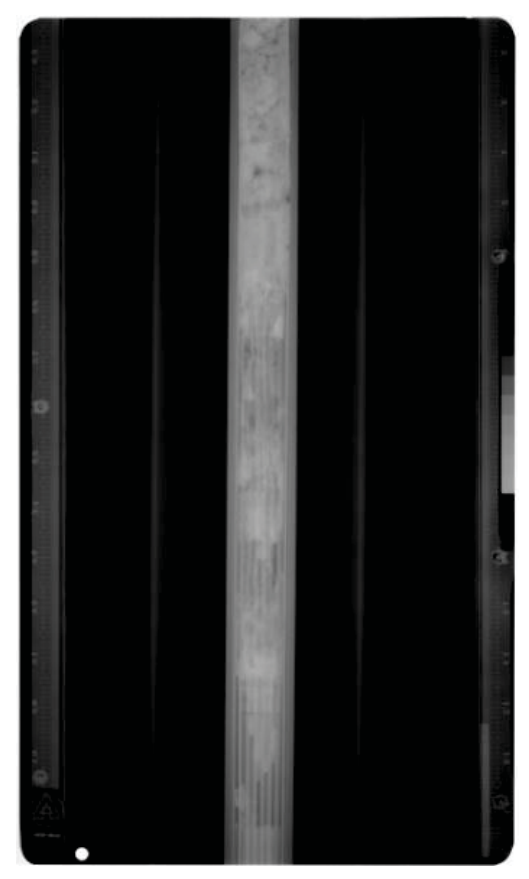

$0^{\circ}$

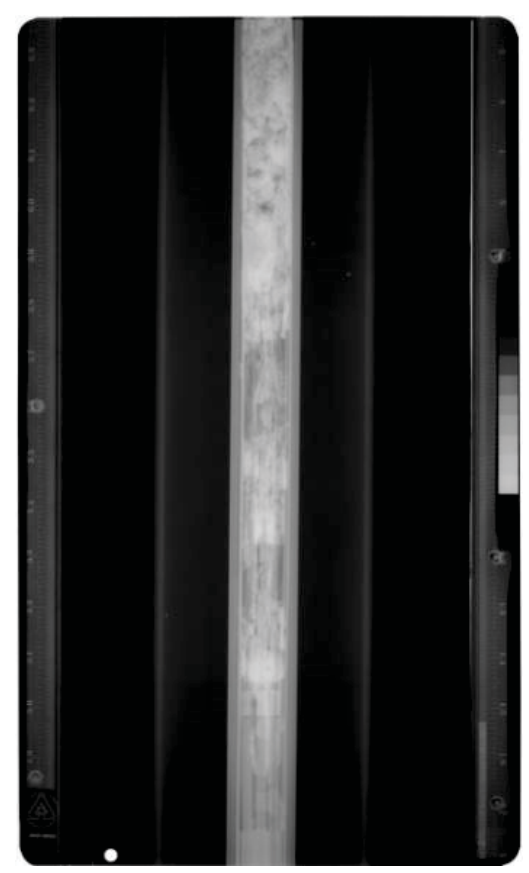

$72^{\circ}$

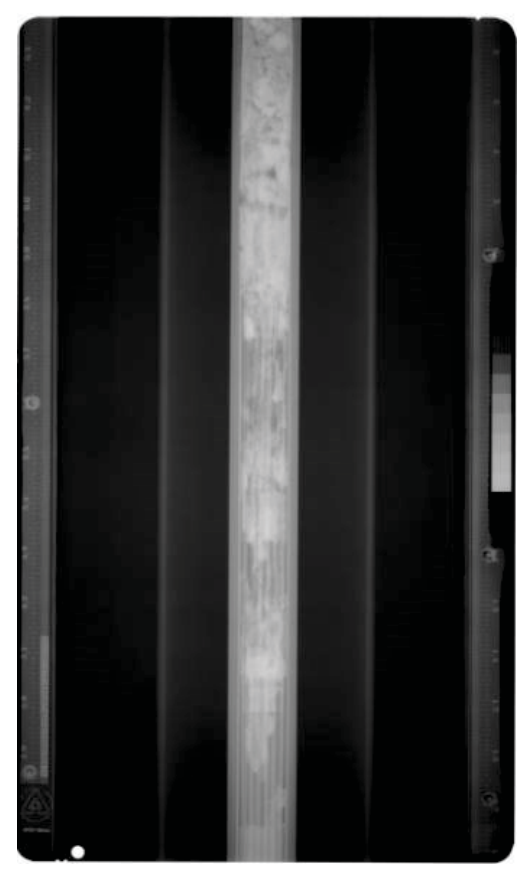

$177.6^{\circ}$

Figure 5.1. Digitized film radiographs of the L07 fuel bundle from three different angles. 


\subsection{Image Processing}

The images of the L07 fuel assembly are processed to improve quality and remove artifacts. Image processing includes manipulation, correction, analysis and interpretation of image to enhance certain features while suppressing unnecessary information.

Image registration is the process of aligning two or more images of the same scene taken at different times, different viewpoints, and/or by different sensors. ${ }^{15}$ There are two types of image registration, intensity-based and feature-based, and a single registration method will not work for all applications. ${ }^{16}$ The images are first registered by a rotational correction about the axis normal to the image and a translation correction in both horizontal and vertical directions. Feature extraction based Hough transform is used to register all the images of the L07 fuel assembly. Prior to applying Hough transform, a canny edge detection algorithm and a thresholding operation determine a set of pixels in a straight line that defines the boundary of the fuel assembly. Hough transform considers many line passes through a point in spatial domain (Figure 5.2a) by mapping those line's slopes and intercepts into Hough space (Figure 5.2c). If several lines with slopes $\left(m_{1}, m_{2}, m_{3}, \ldots\right)$ and intercepts $\left(c_{1}, c_{2}, c_{3}, \ldots\right)$ pass through a point $\left(x_{i}, y_{i}\right)$ in spatial domain then the general equation of each line is:

$$
y_{i}=\left(m_{1}, m_{2}, m_{3}, \ldots\right) x_{i}+c_{1}, c_{2}, c_{3}, \ldots
$$

In Hough space, the equation for each line is:

$$
c_{1}, c_{2}, c_{3}, \ldots=-x_{i}\left(m_{1}, m_{2}, m_{3}, \ldots\right)+y_{i}
$$

Therefore, all slopes of $\left(x_{i}, y_{i}\right)$ in spatial domain will make one straight line in Hough space. Similarly, slopes of the lines of the other points in spatial domain make their corresponding straight line in Hough space.
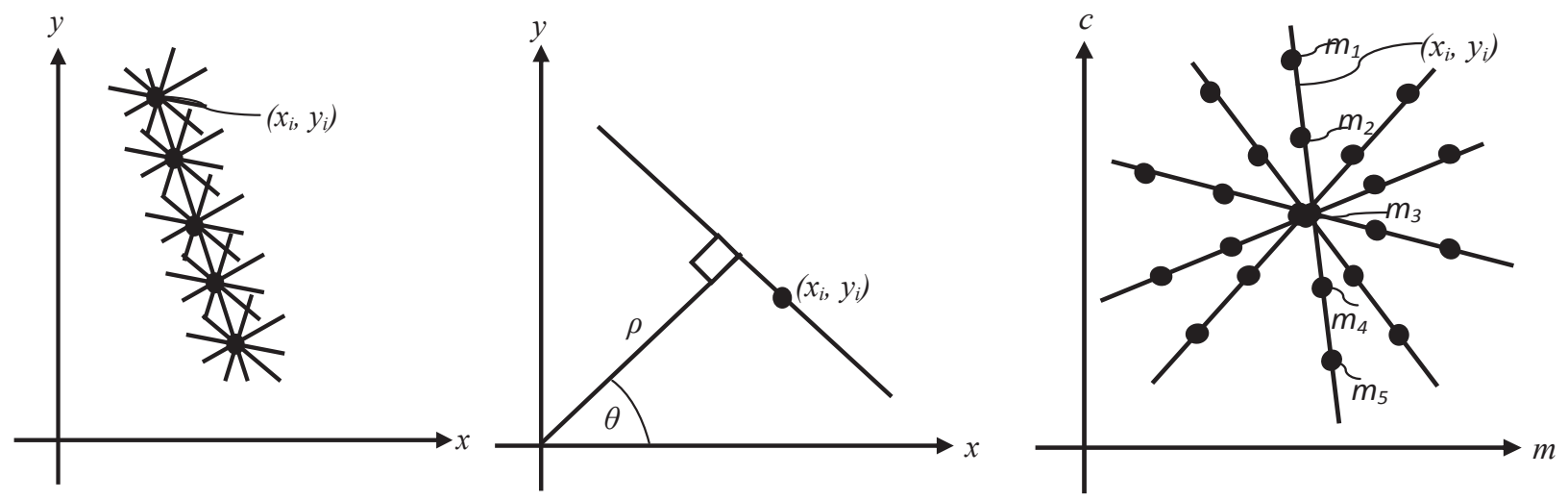

Figure 5.2. Figures describing the Hough transform.

If the points have a common straight line with common slope then they will intersect at a point in Hough space. The slope at the point of intersection will be the slope of the edge of the nuclear fuel. In order to implement the algorithm, polar coordinates are used (Figure 5.2b) instead of Cartesian coordinate where 
each line passing through a point is represented by $\rho$ (perpendicular distance from the origin to the line) and $\theta$ (angle of that line). The polar representation of the line is:

$$
x \cos \theta+y \sin \theta=\rho
$$

Figure 5.3 shows the registered image with a rotation correction to straighten the image of the specimen. The background is not necessary for reconstruction, so the images are cropped to remove the background prior to reconstruction. Figure 5.4 shows the cropped fuel bundle from the bottom of the assembly. After performing the cropping operation the images have to be registered.

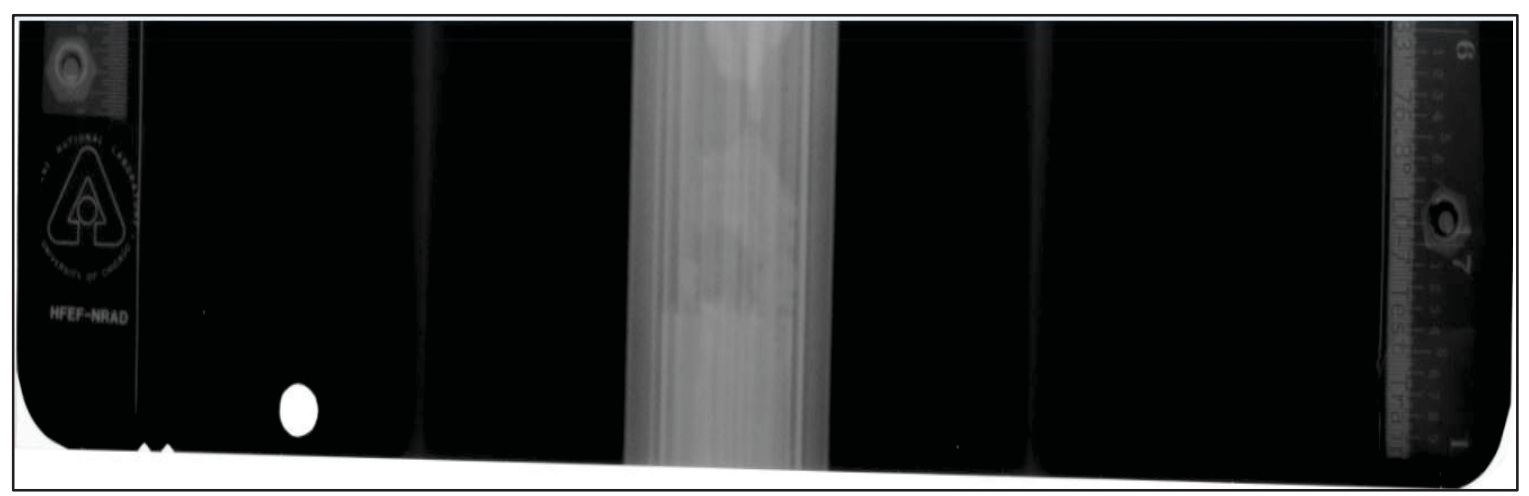

a) Registered image before rotation correction.

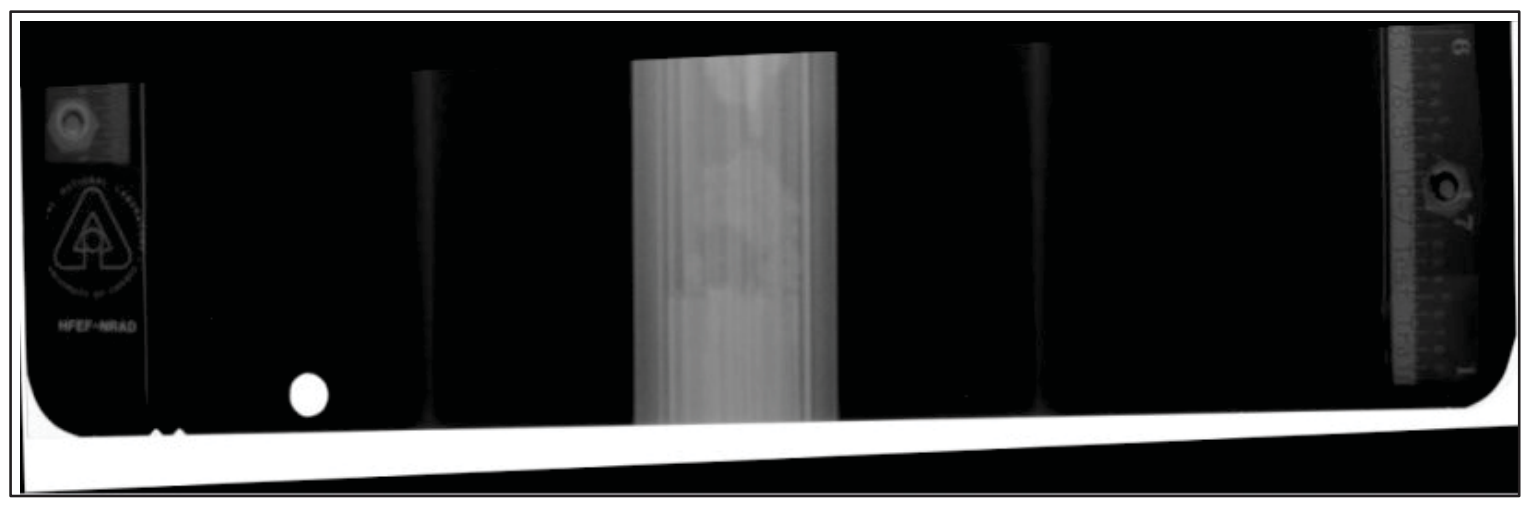

b) Registered image after rotation correction.

Figure 5.3. Rotation-corrected registered image.
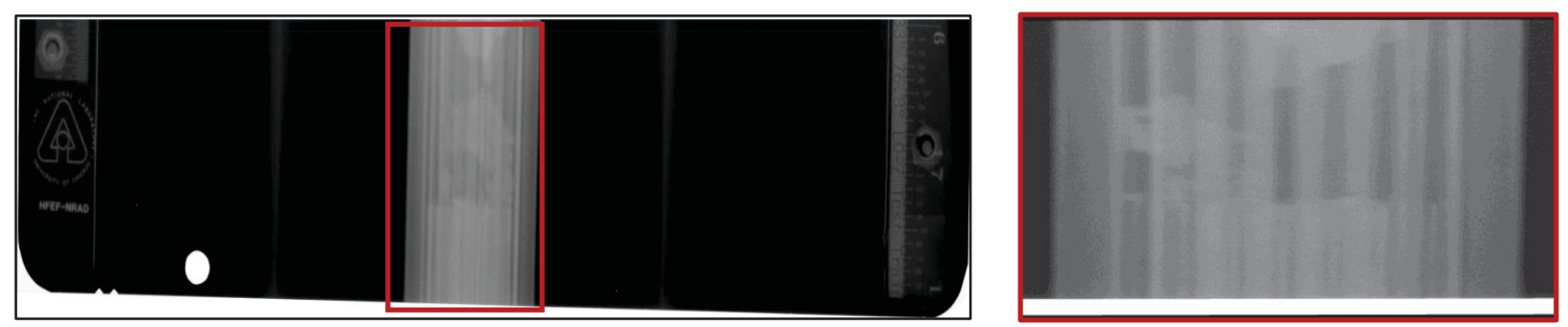

Figure 5.4. Cropped L07 fuel bundle. 
A 2D phase correlation determines a straight-forward estimation of rigid translational motion between two images, which is based on Fourier shifting property. Fourier transform, which represents images in frequency domain, results in a phase difference between two images if they are shifted in spatial domain. If two images, $g(x, y)$ and $h(x, y)$, have simple translational shift $a$ in horizontal direction and $b$ in vertical direction, the Fourier transform of the two images is:

$$
H(u, v)=G(u, v) e^{-i(a u+b v)}
$$

The normalized cross power spectrum is:

$$
L(u, v)=\frac{G(u, v) H(u, v)^{*}}{\left|G(u, v) H(u, v)^{*}\right|}=e^{-i(a u+b v)}
$$

Taking the inverse of $L(u, v)$ leads to a Dirac delta function and its peak gives the shift between the two images. The shift is calculated and the images translated, as shown in Figure 5.5.

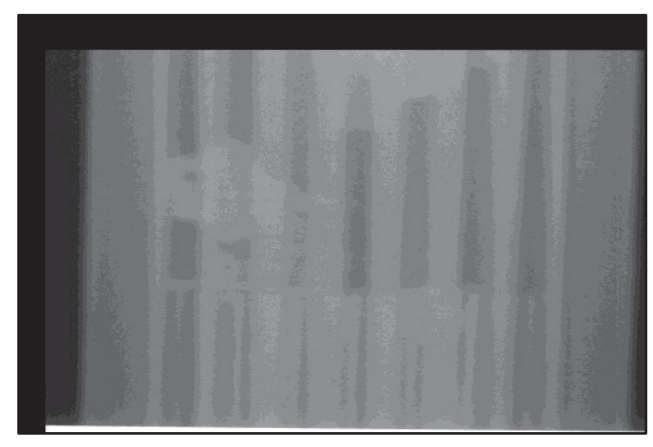

a) Before translation.

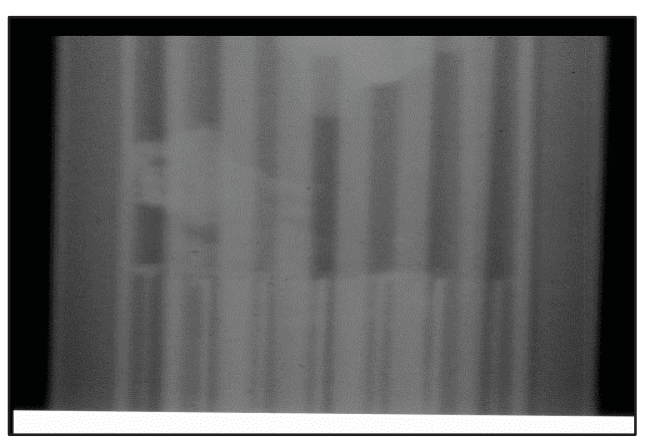

b) After translation.

Figure 5.5. Translation correction in both $\mathrm{x}$ - and $\mathrm{y}$-axis.

A noise reduction algorithm removes the introduction of blurring and artifacts from the registered images. An alpha trimmed mean filter reduces the noise on the images and is also an edge-preserving filter. The filter is a compromise between mean and median filter and gives local information when the Gaussian data contains outliers. This non-linear filter reduces Gaussian noise as well as "salt-and-pepper" noise. The alpha trimmed mean filter $\left(m_{n}\right)$ is defined as: ${ }^{17}$

$$
m_{n}(i ; \alpha)=\frac{1}{n-2[\alpha n]} \sum_{j=[\alpha n]+1}^{n-[\alpha n]} x_{j}(i),
$$

where $\mathrm{x}_{1}(\mathrm{i}) \leq \mathrm{x}_{2}(\mathrm{i}) \leq \mathrm{x}_{3}(\mathrm{i}) \leq \ldots \leq \mathrm{x}_{\mathrm{n}}(\mathrm{i})$ is an ordered set where $x_{l}(i)$ is the minimum and $x_{n}(i)$ the maximum, signal value. The $\alpha$ indicates the percentage of the signal to be trimmed. If $\alpha$ is around 0.5 , the filter is close to a median filter, and if $\alpha$ approaches zero, the filter becomes a moving-average filter. 


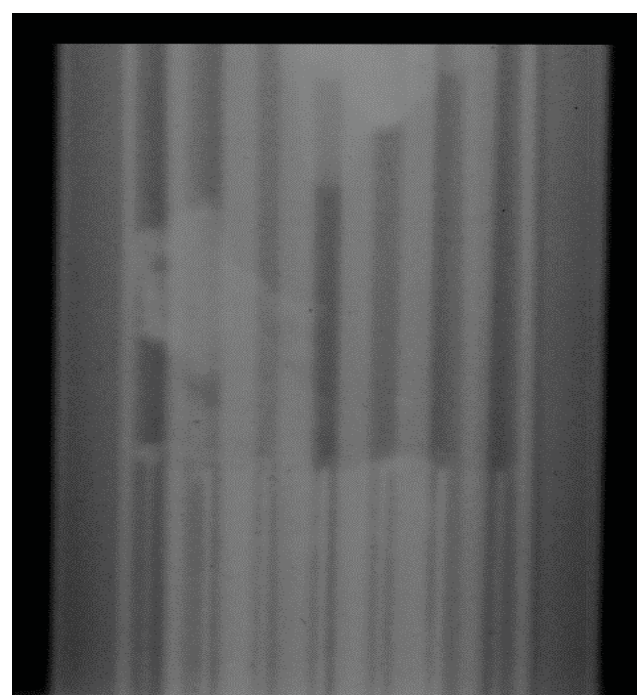

a) Original image.

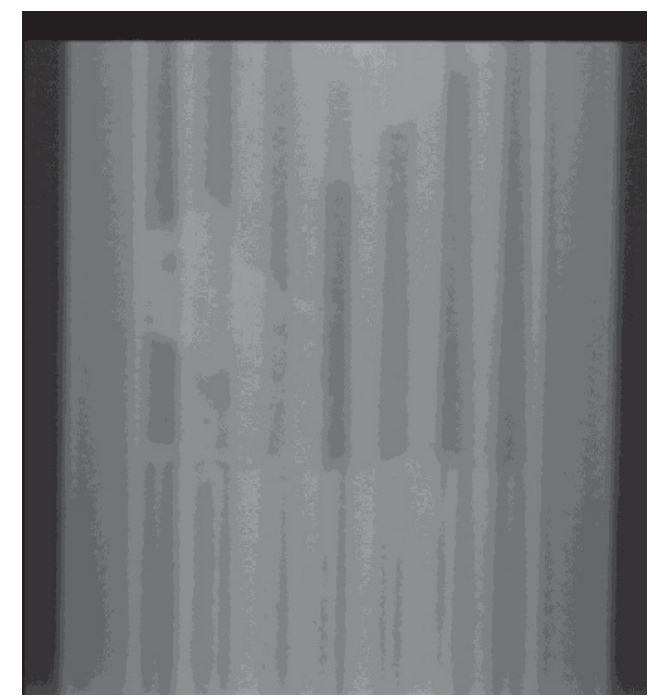

a) Image after noise corrections.

Figure 5.6. Noise reduction using alpha trimmed mean filtering.

The digitizer delivers the image in 16-bit grayscale, so the pixel values vary between 0 and 65535 . Figure 5.7 shows the dynamic range corrected and cropped from the bottom to avoid the white region in the bottom. The images are scaled to cover the range $0-65535$ by performing the following arithmetic operation to utilize the full 16-bit dynamic range:

$$
I^{\prime}(x, y)=65535 \times \frac{I(x, y)-I(x, y)_{\min }}{I(x, y)_{\max }}
$$

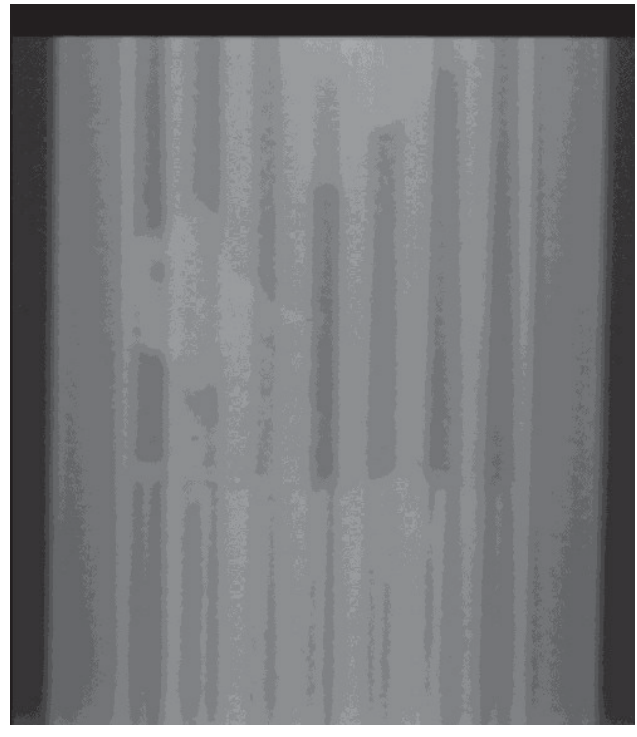

a) Original image.

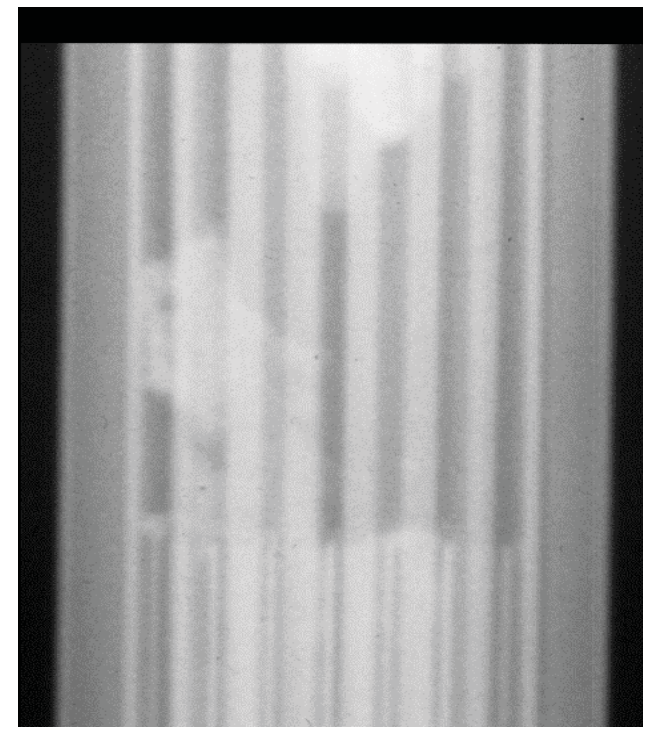

a) Image after noise corrections.

Figure 5.7. Dynamic range corrected and cropped from bottom. 


\subsection{Image Reconstruction}

Computed tomography produces a 3D reconstruction from a set of 2D radiographs of a specimen. There are a number of algorithms for computed tomography, and this work uses a FBP algorithm. While FBP is faster and requires less memory than some advanced reconstruction algorithms, it is sensitive to noise and requires complete $180^{\circ}$ data. An iterative reconstruction algorithm is developed that minimizes the sensitivity to noise and preserves the edges.

\subsubsection{Filtered Back Projection}

Filtered back-projection is a well-known technique for parallel beam image reconstruction algorithm that involves the following steps:

1. Find the 1D Fourier transform for projections at each angle.

2. Apply filters to the result obtained from Step 1 .

3. Find the inverse Fourier transform of the result obtained from Step 2.

4. Back-project the result obtained from Step 3 to spatial domain.

The filter used in FBP is a high pass filter to reduce blurring caused by the $1 /|r|$ non-uniform weighting function, where:

$$
|r|=\sqrt{r_{x}^{2}+r_{y}^{2}}
$$

in the 2D Fourier plane. Applying various filters (e.g. Ram-Lak, Hamming, Han) reduces high frequency (i.e. noise) but increases the blurring effect. Therefore, a perfect filter is difficult to construct without prior knowledge of the type of noise. Figure 8 shows the FBP result of a cross section of L07 fuel using a Hamming filter.

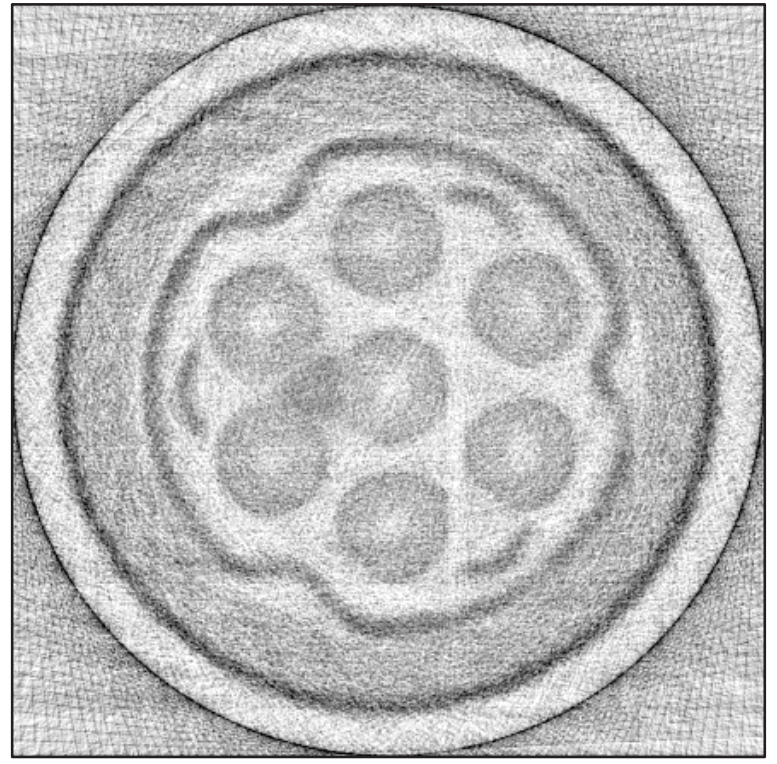

Figure 5.8. Reconstruction using FBP algorithm with a Hamming filter. 


\subsubsection{Iterative Reconstruction}

An iterative reconstruction algorithm developed as part of this work produces a better-quality image than the image obtained by conventional FBP. The FBP algorithm utilizes an integral equation, but the iterative algorithm uses algebraic equation that models complex imaging geometry and imaging physics more realistically. Another disadvantage of FBP is that it cannot model noise, and the noise control is achieved by frequency windowing.

Maximum likelihood expectation maximization (ML-EM) algorithm is used to reconstruct the L07 fuel bundle because it can model noise by minimizing an objective function. The ML-EM algorithm uses prior knowledge about the projection data to make the iterative algorithm more stable. The FBP reconstructed image is used as an initial guess for the ML-EM algorithm. The general reconstruction algorithm for the ML-EM is:

$$
x_{j}^{\text {new }}=\frac{x_{j}^{\text {current }}}{\sum_{j=1}^{N} a_{i j}} \sum_{i=1}^{M} a_{i j} \frac{P_{i}}{\sum a_{i j} x_{j}},
$$

where $P_{i}$ is the measured projection, $x_{j}^{\text {new }}$ is the value of the pixel $x_{j}$ after $(n+1)^{\text {th }}$ iteration, $x_{j}^{\text {current }}$ is the value of the pixel $x_{j}$ after $n^{\text {th }}$ iteration, $\Sigma a_{i j}$ is the weight of the contribution of pixel $x_{j}$ to the projection $P_{i}$ (backprojection of constant, 1, in the image domain), and $M \times N$ is the image size. The ML-EM algorithm has two steps:

1. $E$ (expectation) step: estimation of observed data from current estimate of the model parameters and observed data.

2. $M$ (maximization) step: computation of the maximum-likelihood estimate of the model parameters using estimated observed data.

Convergence is an important criterion for any iterative algorithm. Each iteration of the EM algorithm includes a step to determine the likelihood of convergence. If the likelihood function converges, it returns to the E-step for next iteration.

ML-EM algorithm reconstructs images from limited data but the result is not useful enough. A regularization parameter is employed to produce a smooth image with sharp edges. Several methods (e.g. Bayesian and total variation) are used as a regularization parameter. Total variation (TV) based ML-EM algorithm is used to reconstruct L07 fuel bundle. TV measures how the signal changes between signal values, and thus measures the change of signal if any information is missing in between two rotation angles. In $1 \mathrm{D}$, the $\mathrm{TV}$ of $N$ point signal is defined as:

$$
T V(x)=\sum_{i=1}^{N-1}\left|x_{i+1}-x_{i}\right|
$$

In $2 \mathrm{D}$ form, it can be written as:

$$
T V(x, y)=\sum_{i, j=1}^{N-1}\left|x_{i+1, j}-x_{i, j}\right|+\left|x_{i, j+1}-x_{i, j}\right|
$$

Including the TV algorithm with the ML-EM algorithm produces a TV-ML-EM algorithm that can be written as: ${ }^{18}$ 


$$
x_{j}^{\text {new }}=\frac{x_{j}^{\text {current }}}{\sum_{j=1}^{N} a_{i j}+\beta \frac{\partial T V(X)}{\partial x_{j}}} \sum_{i=1}^{M} a_{i j} \frac{P_{i}}{\sum a_{i j} x_{j}}
$$

where,

$$
\frac{\partial T V(X)}{\partial x_{k, l}}=\frac{x_{k, l}-x_{k-1, l}}{\sqrt{\left(x_{k, l}-x_{k-1, l}\right)^{2}+\left(x_{k-1, l+1}-x_{k-1, l}\right)^{2}+\delta^{2}}}+\frac{x_{k, l}-x_{k, l-1}}{\sqrt{\left(x_{k+1, l-1}-x_{k, l-1}\right)^{2}+\left(x_{k, l}-x_{k, l}\right)^{2}+\delta^{2}}}-\frac{x_{k+1, l}+x_{k, l+1}-2 x_{k, l}}{\sqrt{\left(x_{k+1, l}-x_{k, l}\right)^{2}+\left(x_{k, l}+1-x_{k, l}\right)^{2}+\delta^{2}}},
$$

where $x_{k, l}$ is a pixel of image $x$ and $\delta$ is a penalized parameter. The algorithm can be described in the following steps:

1. Find the forward projection of the FBP reconstructed image.

2. Calculate the ratios of the sinogram and the forward projection.

3. Backprojection of the ratios.

4. Backprojection of a constant, 1.

5. Calculate pixel-by-pixel ratio of the back-projected image obtained from step 3 and step 4.

6. Divide the TV regularization parameter from the pixel-by-pixel ratio obtained in step 5.

7. Point-by-point multiplication of the FBP image with the result obtained in step 6.

Figure 5.9 shows the reconstruction of the L07 assembly using TV-ML-EM algorithm with one and two iterations.

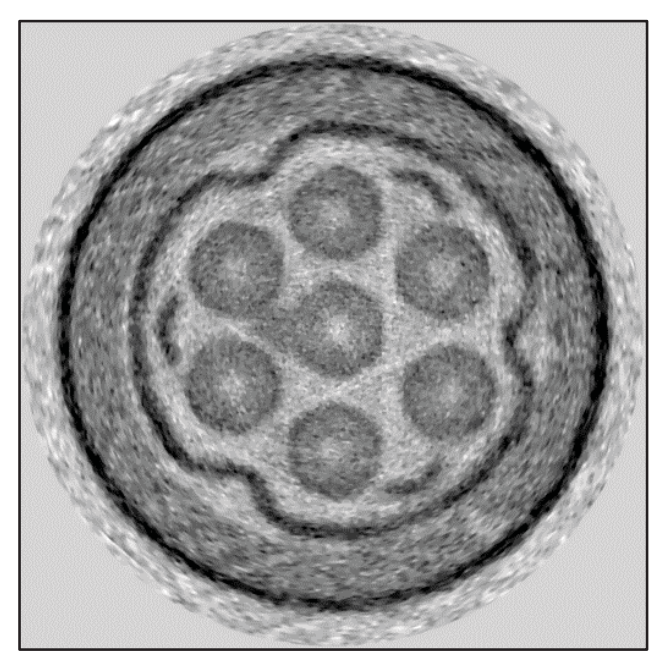

a) TV-ML-EM using one iteration.

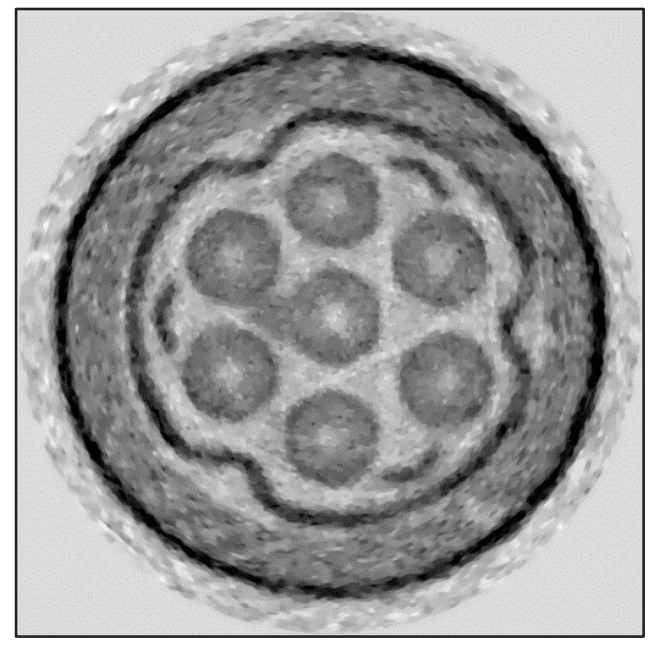

b) TV-ML-EM using two iterations.

Figure 5.9. TV-ML-EM algorithm using one and two iterations.

\subsection{Recommendation for the AFIP-7 Experiment}

Missouri S\&T has received a mockup of AFIP-7 for proof of principle of tomography and tomosynthesis. Neutron radiographs will be produced at the Missouri S\&T reactor and will perform image processing and reconstruction with the images. A recommendation about the angular distribution of the radiographs that result in a reconstruction with the highest level of detail will also be provided. The reconstruction will be analyzed for gap widths, gross geometric defects, and local defects. Missouri S\&T will determine the spatial resolution of the reconstruction using community accepted methods, and determine the types of detail that are expected to be seen in the resulting reconstruction. 


\section{COMPUTED TOMOGRAPHY AT REAL TIME TOMOGRAPHY}

Recent advances over the last decade have already made X-ray computed tomography (XCT) an increasingly important tool in science and engineering. Other emerging techniques such as neutron computed tomography (NCT) or 3-D imaging using infrared (IR) and longer wavelength radiation are only beginning to be exploited but have unique advantages and high potential for the future. For example, NCT combines attractive penetration depths with effective imaging of light elements when compared to $\mathrm{XCT}$, attractive features for getting adequate image contrast in organic systems, multiphase fluid systems, hydrogen containing systems, and, in this case, high density metals; however, development has been limited due to a lack of neutron sources.

The INL currently uses neutron radiography to evaluate irradiation induced changes in the monolithic fuel meats of individual fuel plates. The GTRI program is interested in expanding this capability by developing a neutron tomography capability that will allow 3D examination of irradiated multi-plate elements at the Neutron Radiography Reactor (NRAD). The program is working with university partners to accelerate this development. Real Time Tomography (RTT) has extensive experience in reconstructing 3D images from a wide variety of imaging systems, including breast and body image acquisition systems, and security screening systems. With expertise in tomosynthesis image reconstruction, RTT understands how the acquisition system and setup can affect the image quality of the resultant images and how to improve the image quality through careful system design. RTT has also developed a variety of imaging techniques, algorithms and software that have employed to render the optimal image quality. INL, OSU, and Missouri S\&T will collaborate with RTT on the design of INL's Neutron Imaging System for optimal image quality.

Previous work has demonstrated the feasibility of using computed tomography for non-destructive imaging of a TREAT fuel assembly using neutron radiographs. ${ }^{19}$ However, the acquisition of the full $180^{\circ}$ of projection images is expensive in terms of man-hours and reactor time. A possible alternative would be to utilize a tomosynthesis imaging approach. Tomosynthesis is a form of computed tomography in which projection images over a limited angular range are acquired and reconstructed into tomographic images. Tomosynthesis offers the advantage of requiring far fewer projections, which can offer a significant time and cost savings.

The trade-off of tomosynthesis is that spatial resolution in the reconstructed images is anisotropic. Out-ofplane spatial resolution (in the $z$-direction, along the ray from the source to the detector in the central projection) is poorer with tomosynthesis due to incomplete spatial sampling in the limited angle acquisition. However, in-plane lateral resolution can be quite good and is generally at least equivalent to the detector resolution. With a suitable acquisition geometry, super-resolution is possible, which can allow in-plane reconstruction resolution which is finer than the detector resolution. ${ }^{20}$

The following subsections outline and summarize the tasks that were performed during the funded base period by Real Time Tomography. Three separate image reconstructions of fuel assemblies were performed with the following:

1. Digitized radiographs of a TREAT assembly that were manually registered by RTT (Section 6.1).

2. Cropped field-of-view digitized TREAT radiographs which were semi-automatically registered by Missouri S\&T (Section 6.2).

3. Digital projection images of an AFIP-7 assembly acquired on a prototype digital detector from Oregon State University (OSU) (Section 6.3).

The scope of the tasks covered image reconstruction, image analysis and recommendations. 


\subsection{TREAT Radiographs with Manual Registration}

Prior to delivery of AFIP-7 images from OSU, INL provided digitized film radiographs of the TREAT fuel assembly to RTT to demonstrate initial proof of concept of neutron tomosynthesis imaging. These film radiographs were previously acquired for a series of experiments conducted in the 1980's to evaluate the use of computed tomography as a standard technique for post-test analysis of fuel melt-downs in the TREAT reactor. ${ }^{19}$

\subsubsection{Methods and Materials}

The film radiographs of the TREAT assembly were digitized by Missouri S\&T using a Genesis NEO S60 scanner with the following specifications:

- Maximum resolution: 600 dpi

- Optical density (DMAX): 4.7

- Bit Depth: 16 bits (65535 shades gray) output

- Sensor: High pixel CCD

The film images were scanned at a 600 dpi resolution to a size of $8401 \times 10801$ pixels. Prior to reconstruction, these digitized projection images were horizontally cropped to $2001 \times 10801$ pixels to remove blank background pixels and to preserve GPU (Graphics Processor Unit) memory. The projection images were also spatially co-registered. However, due to a lack of easily segmented fiducial markers in the images that could be used for automated localization, the registration was performed manually. For this reason, only 12 projection images covering a $26.4^{\circ}$ angular range were spatially co-registered and used for this reconstruction test.

To reconstruct the digitized images, RTT's Bryant 3D image reconstruction software was used. The Bryant software was developed to use RTT's patented dynamic 3D image reconstruction method which uses a backprojection method that incorporates multi-pass and non-linear operations to optimize the reconstruction. $^{21}$ The TREAT assembly's acquisition geometry was modeled given the available information and parameterized for input to the Bryant software. The reconstruction software was run on a standard Windows PC and GPU-accelerated with Nvidia Quadro video cards.

\subsubsection{Results}

Figure 6.1 shows three longitudinal tomosynthesis image reconstructions (image slices) of the fuel assembly at three different positions. The central image slice (at $0 \mathrm{~mm}$ ) shows three columnar structures corresponding to three damaged central fuel pins. The outer two image slices each have two partially visible columnar structures corresponding to the outer pairs of fuel pins in the hexagonal fuel arrangement. The images were reconstructed to a size of $1024 \times 1024$ pixels, giving an in-plane reconstruction resolution of $0.083 \times 0.45 \mathrm{~mm}$ at the baseline magnification. This reconstruction size was used to maximize the dynamic reconstruction speed to allow interactive visualization and placement of the reconstruction plane position. ${ }^{21}$

Figure 6.2 shows a close-up view of the central longitudinal reconstruction slice. Measurements taken of various structures in the reconstructed image showed good agreement with the fuel assembly specifications (see Table 6.1). Several gap measurements between damaged fuel pins are also illustrated from $0.24 \mathrm{~mm}$ to $5.3 \mathrm{~mm}$. The sub-millimeter measurements were taken on dynamically magnified views at a $1.5 \mathrm{X}$ zoom (resolution of $0.055 \times 0.30 \mathrm{~mm}$ ). The magnified high-resolution reconstruction allowed easier and more accurate placement of the measurement markers for sub-millimeter gap measurements compared to reconstructions at the baseline zoom level. 


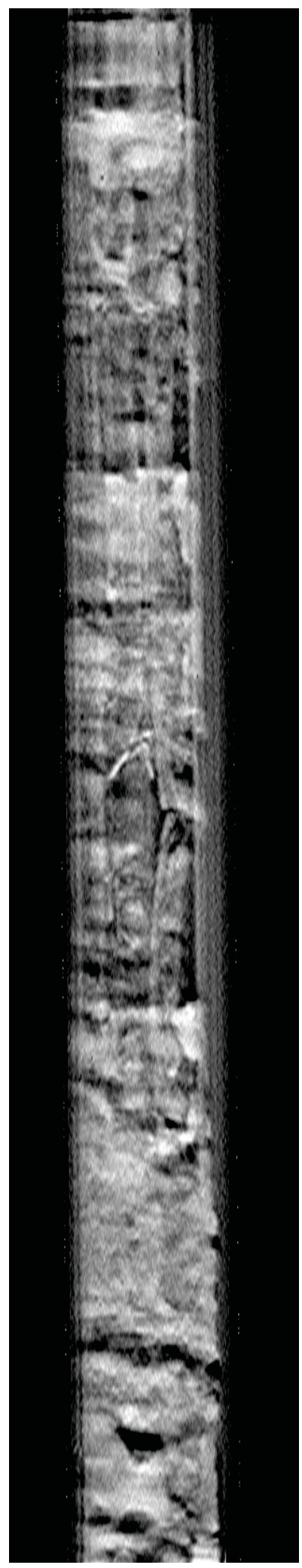

a) Positioned at $-7 \mathrm{~mm}$.

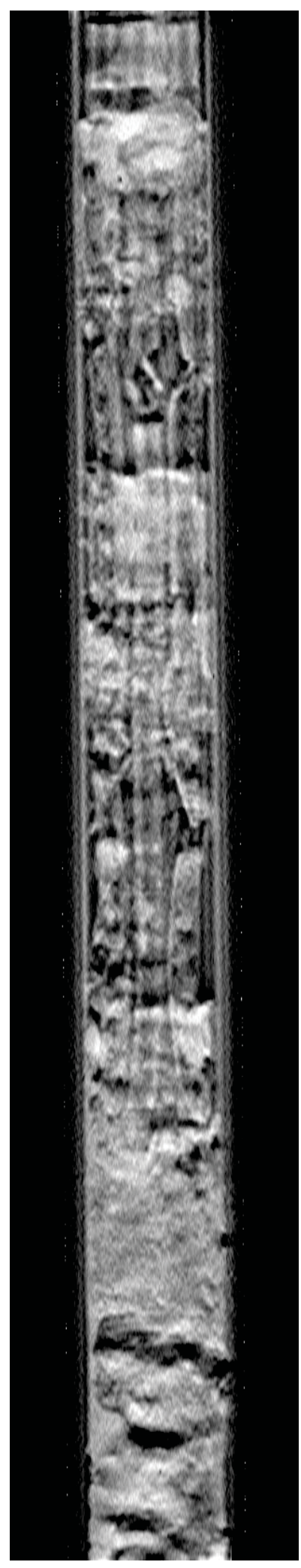

b) Positioned at $0 \mathrm{~mm}$.

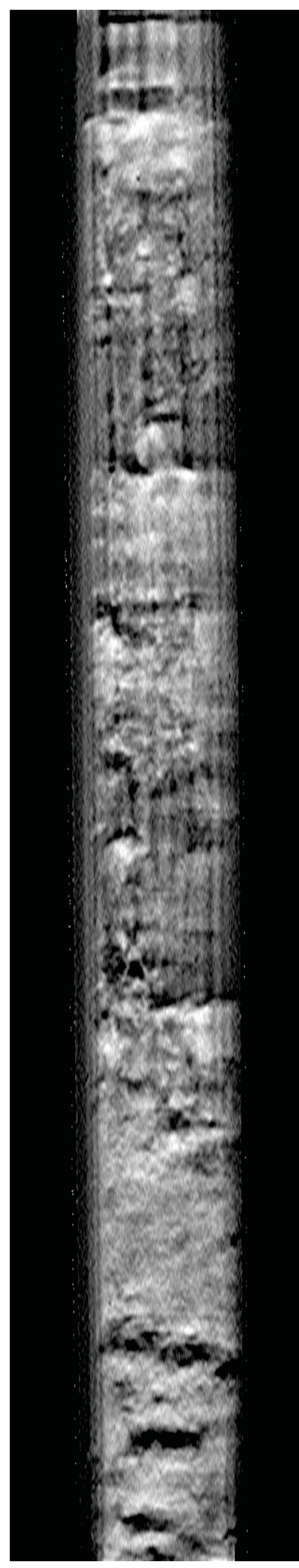

c) Positioned at $7 \mathrm{~mm}$.

Figure 6.1. Twelve projection $26.4^{\circ}$ TREAT longitudinal reconstructions at three positions. 


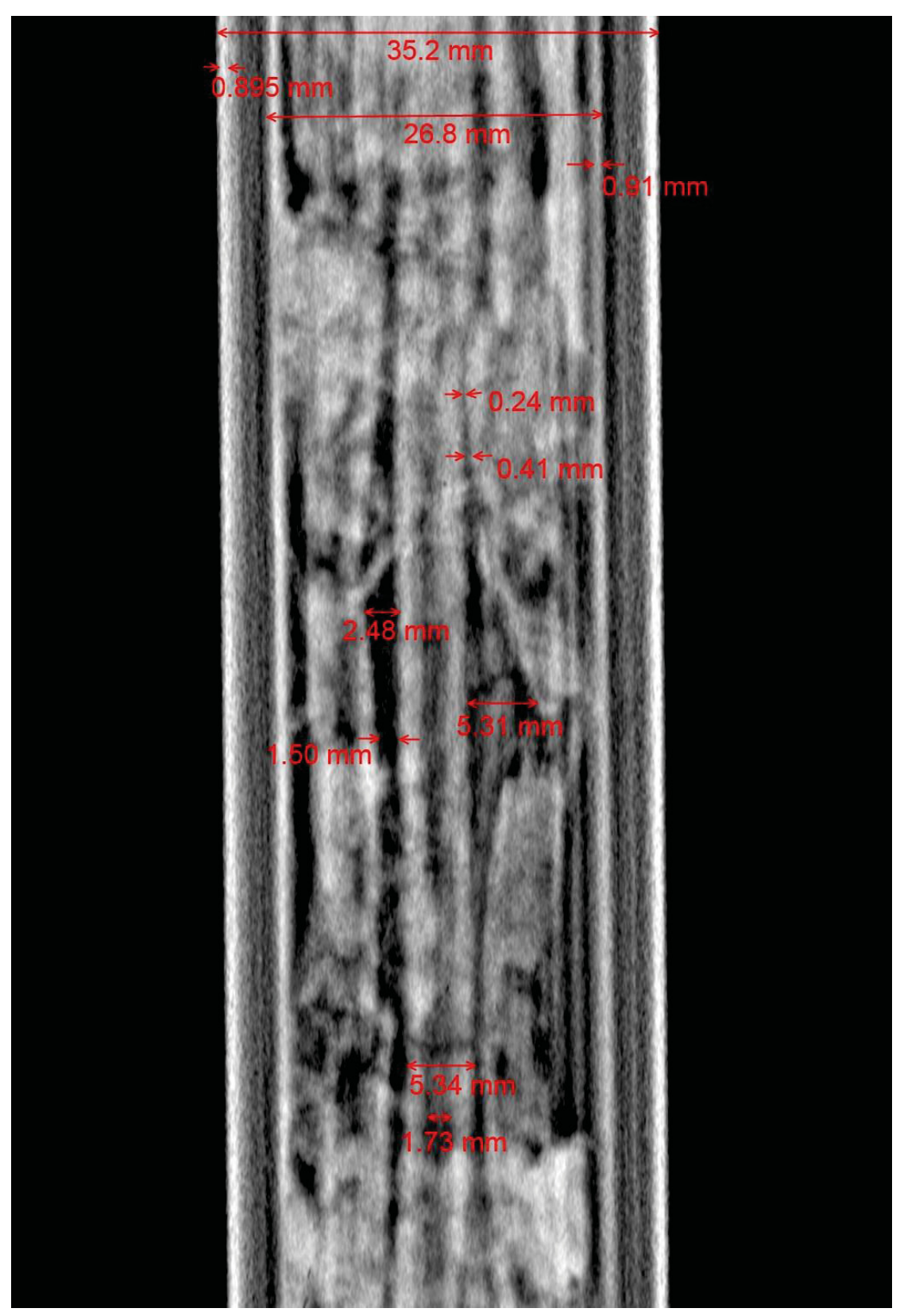

Figure 6.2. Distance measurements on TREAT longitudinal reconstruction with 12 projections over $26.4^{\circ}$.

Table 6.1. Fuel rod actual and reconstruction measurements.

\begin{tabular}{|c|l|c|c|}
\hline \multicolumn{2}{|c|}{ Test Section Dimensions } & $\begin{array}{c}\text { Specified Fuel } \\
\text { Dimensions (mm) }\end{array}$ & $\begin{array}{c}\text { Measured from } \\
\text { Reconstruction (mm) }\end{array}$ \\
\hline \multirow{2}{*}{ Outer Adiabatic Wall } & Outer Diameter & 34.9 & 35.2 \\
\cline { 2 - 4 } & Thickness & 0.89 & 0.895 \\
\hline \multirow{2}{*}{ Tri-Fluted Flow Tube } & Outer Diameter & 26.0 & 26.8 \\
\cline { 2 - 4 } & Thickness & 0.89 & 0.91 \\
\hline \multirow{2}{*}{ Fuel Pellet } & Outer Diameter & $4.95-5.00$ & 5.34 \\
\cline { 2 - 4 } & Annular Hole Diameter & $1.52-1.78$ & 1.73 \\
\hline
\end{tabular}


Initial measurement results suggest that tomosynthesis should have sufficient lateral resolution to visualize the plate spacing $(2.5-3.0 \mathrm{~mm})$ in the AFIP-7 assembly. A calibration phantom with known geometry and dimensions would be necessary to thoroughly characterize the reconstruction resolution. From the measurements that could be made from the fuel assembly reconstructed images, the smallest measurable gap $(0.24 \mathrm{~mm})$ is more than sufficient for plate spacing visualization in the AFIP-7 assembly.

The gap resolution of $0.24 \mathrm{~mm}$ was significantly less than the resolution limit of film (typically in the 0.1 $0.05 \mathrm{~mm}$ range). This may be due to an inability to locate a smaller gap in the reconstructed images; more likely, the less than expected spatial resolution indicates that the imaging process has room for further optimization. Image registration could easily be the most significant source of error, and the first reconstruction parameter to improve. Mis-registrations of the projection images would blur and decrease the spatial resolution of the reconstruction.

\subsection{TREAT Radiographs with Semi Automated Registration}

Another reconstruction test was performed using cropped field of view, semi-automatically registered images supplied by Missouri S\&T of the digitized TREAT film radiographs.

\subsubsection{Methods and Materials}

As with the initial 12 projection reconstruction test discussed in Section 6.1, the projection images (of varying sizes, approximately $6200 \times 1450$ pixels) were cropped to remove extraneous background air pixels. The projections were both horizontally and vertically cropped to a final size of $1592 \times 1402$ pixels. RTT's Bryant 3D software was used to perform both tomosynthesis and CT reconstructions. The reconstructed images were sized at $1024 \times 1024$ pixels, giving a $0.066 \times 0.058 \mathrm{~mm}$ pixel resolution at baseline magnification.

A full set of 76 projection images is acquired over $180^{\circ}$ to compare the image resolution of CT reconstructions to tomosynthesis reconstruction. Figure 6.3 shows reconstructions of transverse views were possible with the full $180^{\circ}$ of images.

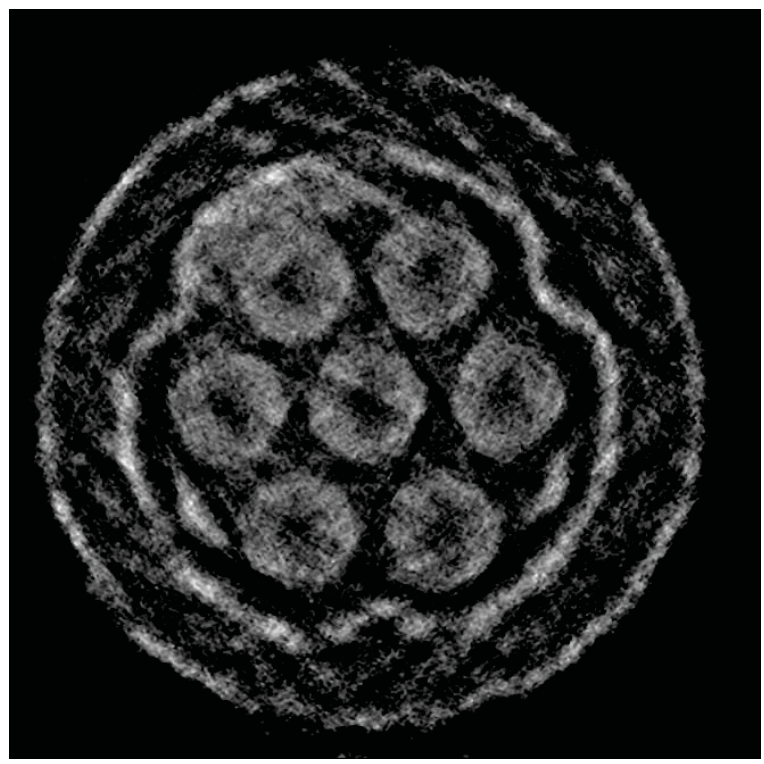

a) Tomosynthesis cross-sectional slice at $\mathrm{z}=0 \mathrm{~mm}$.

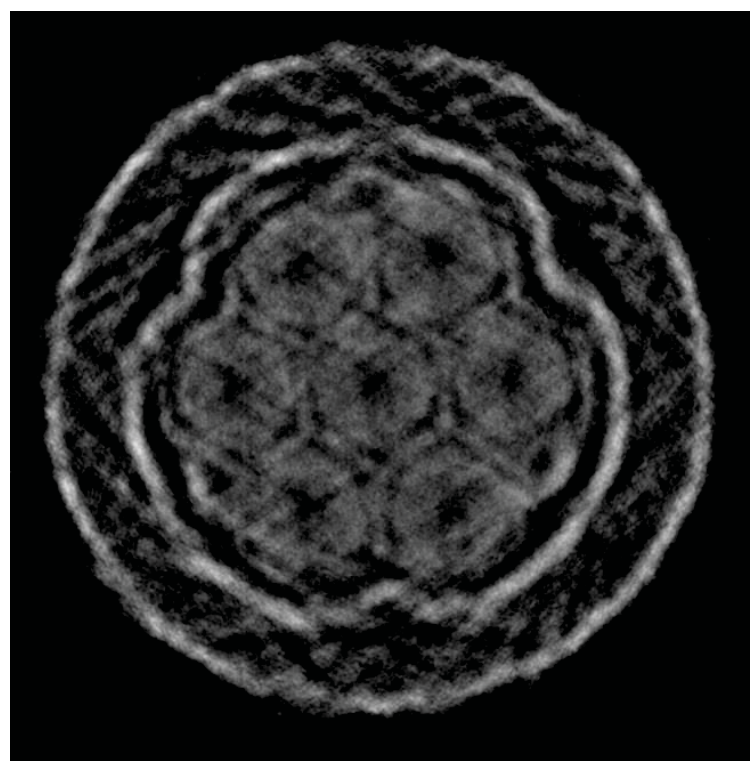

b) A $1 \mathrm{~mm}$ thick slab reconstruction at $\mathrm{z}=10 \mathrm{~mm}$.

Figure 6.3. Transverse TREAT reconstructions from 76 projections at two different axial positions. 
Several longitudinal reconstructions were computed using different combinations of projection angles, number of projections, and azimuth reconstruction plane angles. These reconstruction parameters are listed in

Table 6.2. The reconstruction azimuth angles and plane positions are illustrated in Figure 6.4. Reconstructed image slices for the azimuth angles and plane positions are shown in Figure 6.5 to Figure 6.8 .

Table 6.2. Tomosynthesis reconstruction parameters.

\begin{tabular}{|c|c|c|c|}
\hline $\begin{array}{c}\text { Azimuth } \\
\text { Angle } \\
\text { (degrees) }\end{array}$ & $\begin{array}{c}\text { Number of } \\
\text { Projections }\end{array}$ & $\begin{array}{c}\text { Angular Range } \\
\text { (degrees) }\end{array}$ & $\begin{array}{c}\text { Reconstruction } \\
\text { Plane } \\
\text { z-Position (mm) }\end{array}$ \\
\hline \multirow{3}{*}{0} & 76 & 180 & $0.0,7.0$ \\
& 29 & 67.2 & $0.0,7.0$ \\
& 25 & 57.6 & $0.0,7.0$ \\
& 21 & 48 & $0.0,7.0$ \\
& 15 & 33.6 & $0.0,7.0$ \\
& 15 & 67.2 & $0.0,7.0$ \\
& 76 & 180 & $0.0,3.5$ \\
& 29 & 67.2 & $0.0,3.5$ \\
& 25 & 57.6 & $0.0,3.5$ \\
& 21 & 48 & $0.0,3.5$ \\
& 15 & 33.6 & $0.0,3.5$ \\
& 15 & 67.2 & $0.0,3.5$ \\
\hline
\end{tabular}

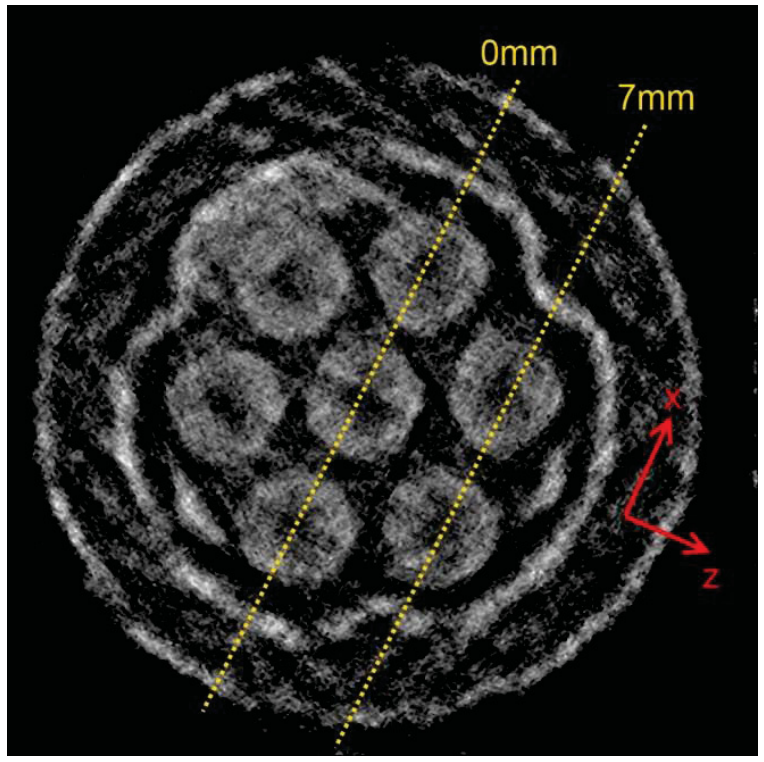

a) Azimuth angle of $0^{\circ}$.

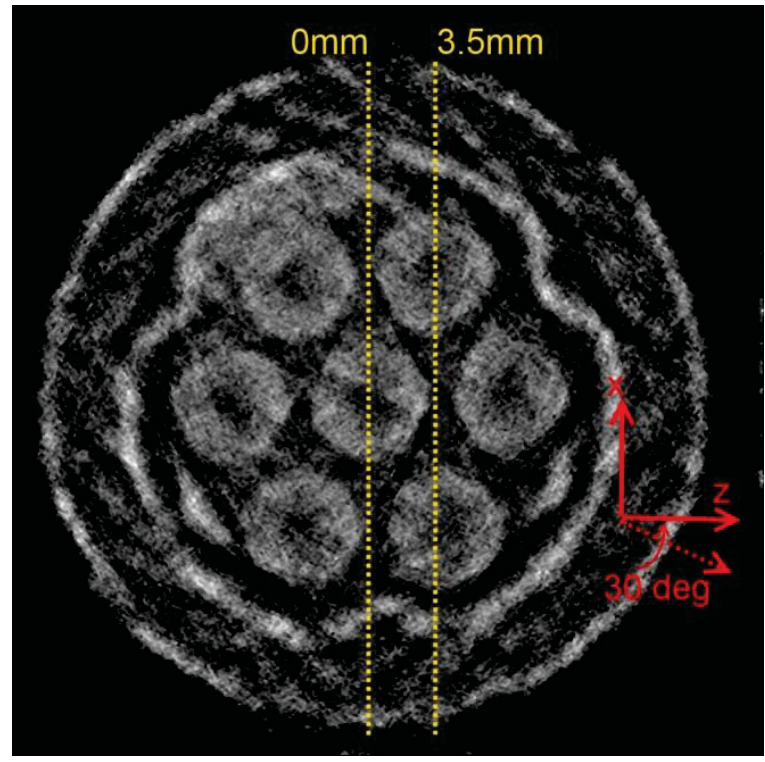

b) Azimuth angle of $30^{\circ}$.

Figure 6.4. Reconstruction plane positions at two azimuth angles. 


\subsubsection{Results}

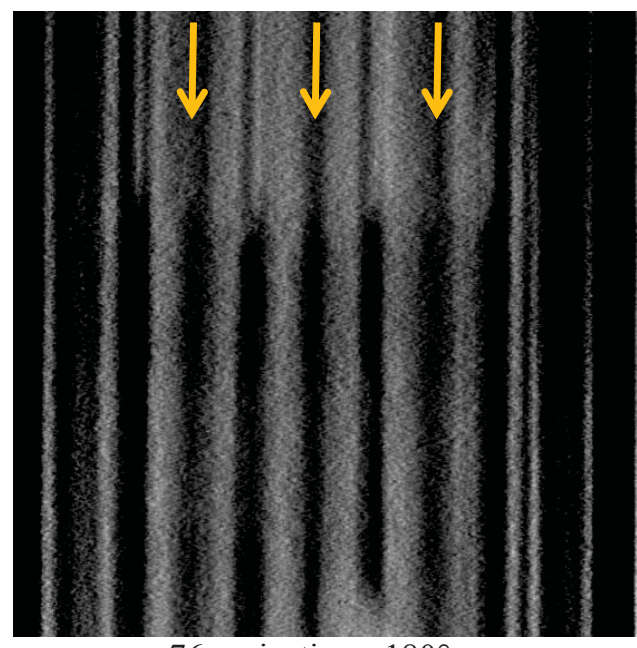

76 projections, $180^{\circ}$

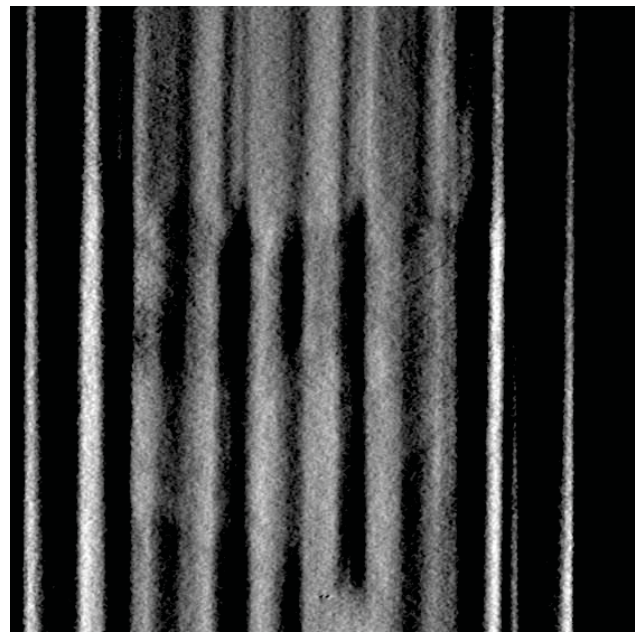

25 projections, $57.6^{\circ}$

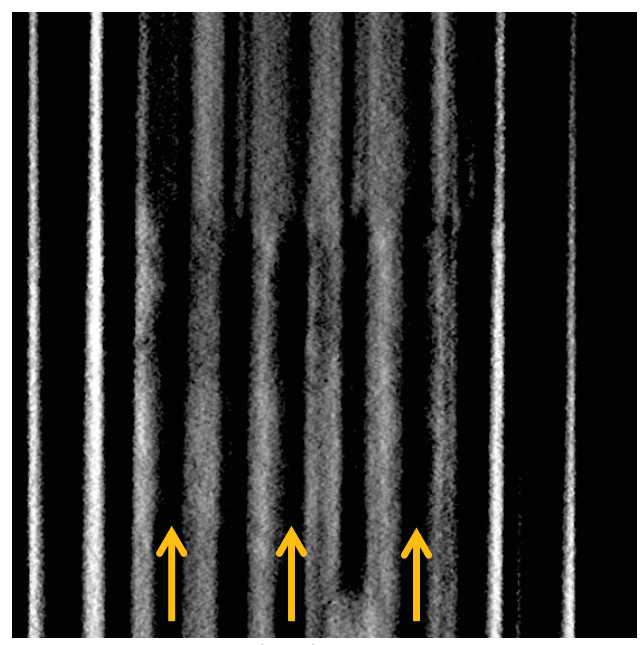

15 projections, $33.6^{\circ}$

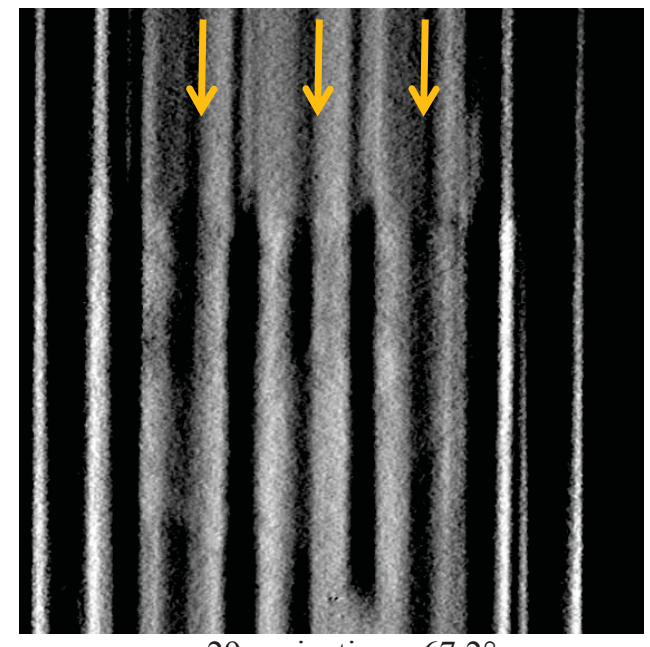

29 projections, $67.2^{\circ}$

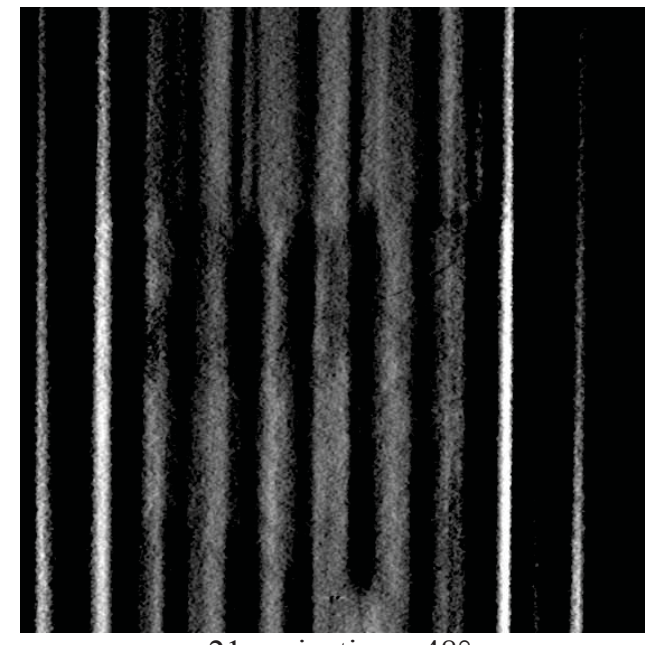

21 projections, $48^{\circ}$

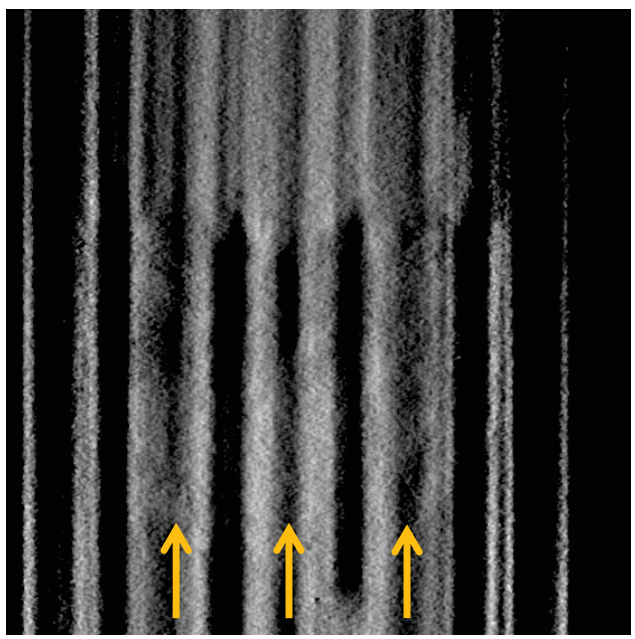

15 projections, $67.2^{\circ}$

Figure 6.5. Longitudinal reconstructions at azimuth $=0^{\circ}$ and $\mathrm{z}=0 \mathrm{~mm}$. 
Arrows denote position of columns corresponding to fuel.

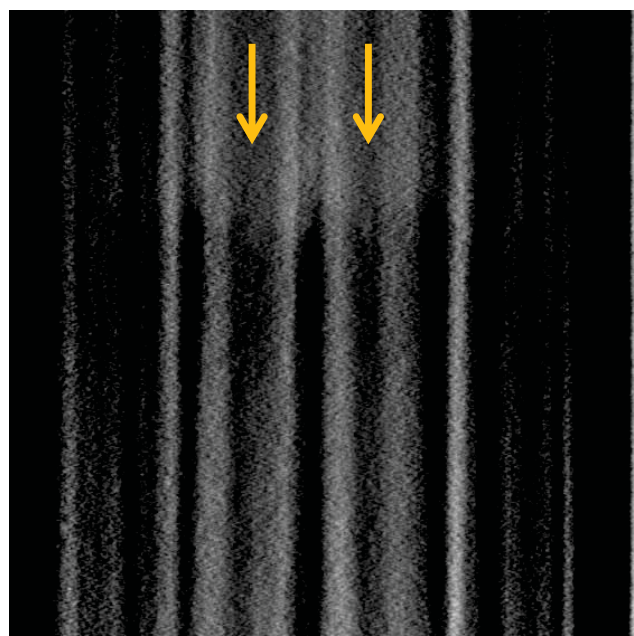

76 projections, $180^{\circ}$

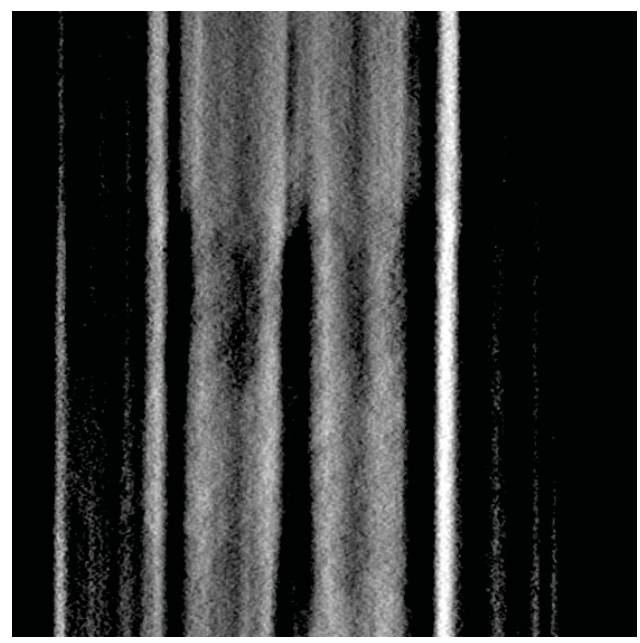

25 projections, $57.6^{\circ}$

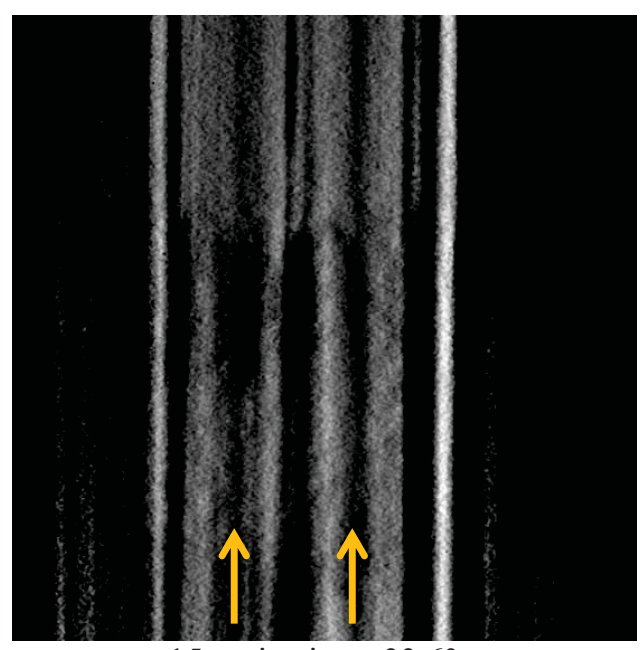

15 projections, $33.6^{\circ}$

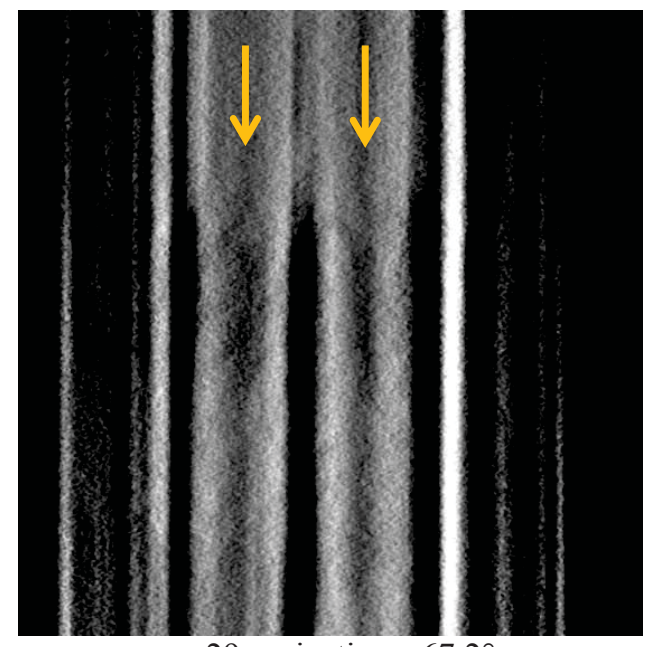

29 projections, $67.2^{\circ}$

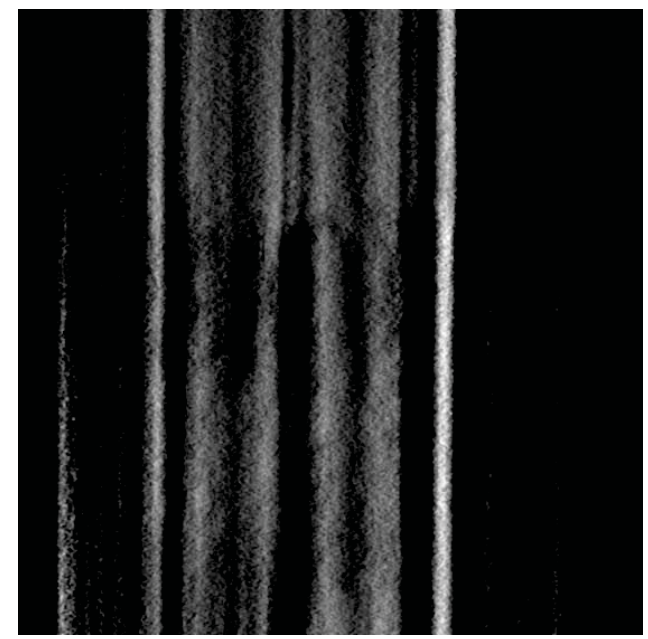

21 projections, $48^{\circ}$

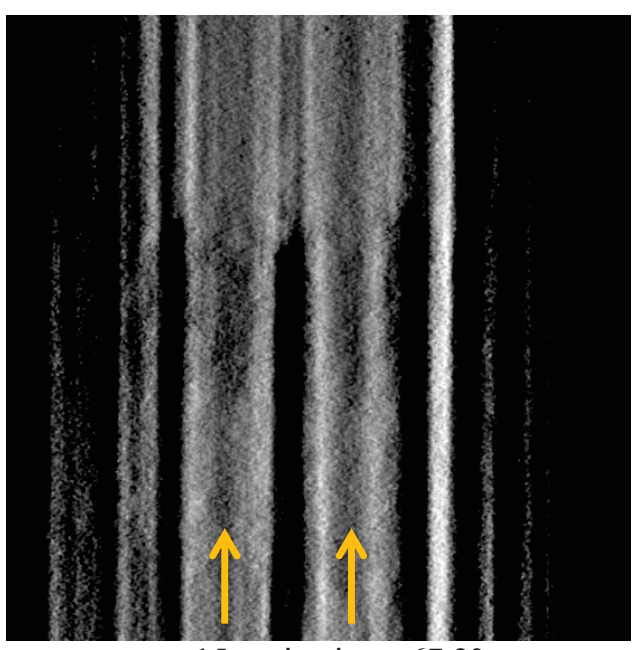

15 projections, $67.2^{\circ}$

Figure 6.6. Longitudinal reconstructions at azimuth $=0^{\circ}$ and $\mathrm{z}=7 \mathrm{~mm}$.

Arrows denote position of columns corresponding to fuel. 


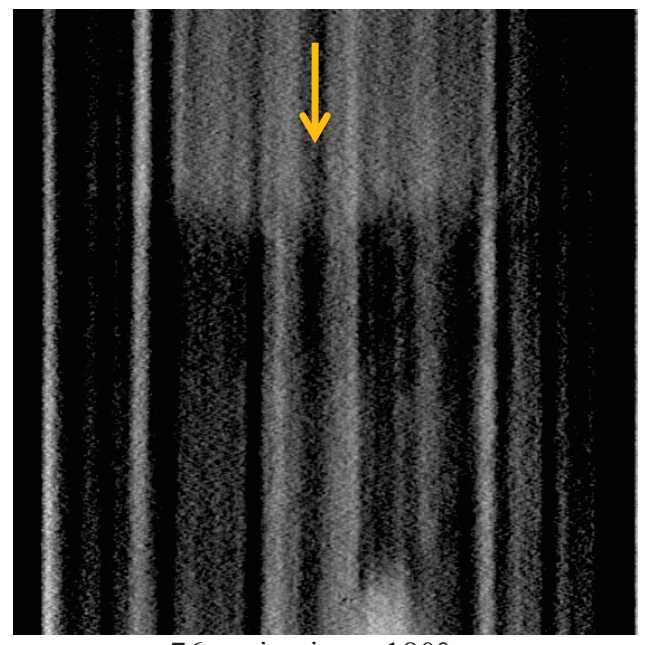

76 projections, $180^{\circ}$

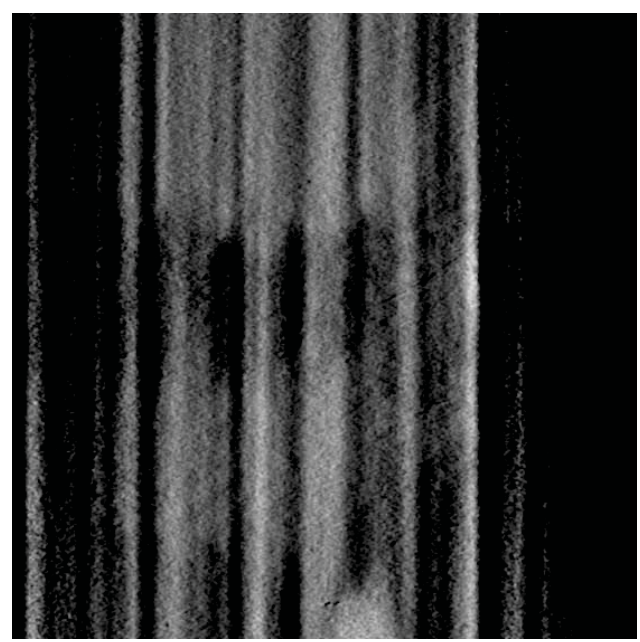

25 projections, $57.6^{\circ}$

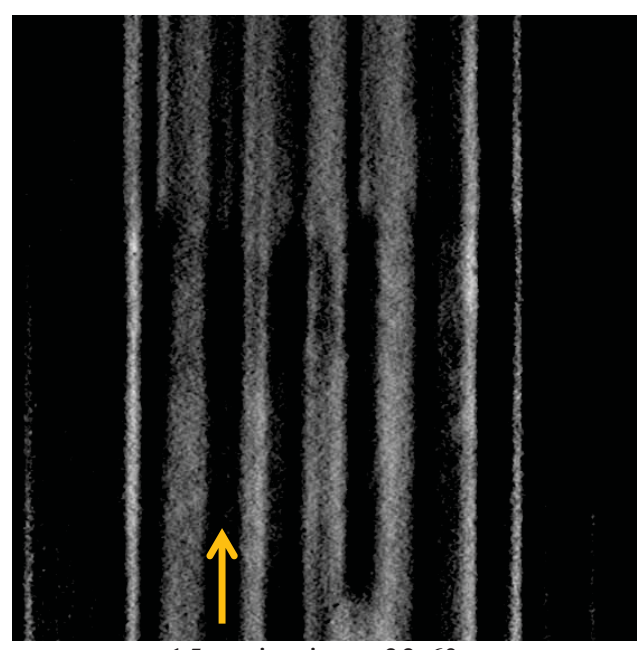

15 projections, $33.6^{\circ}$

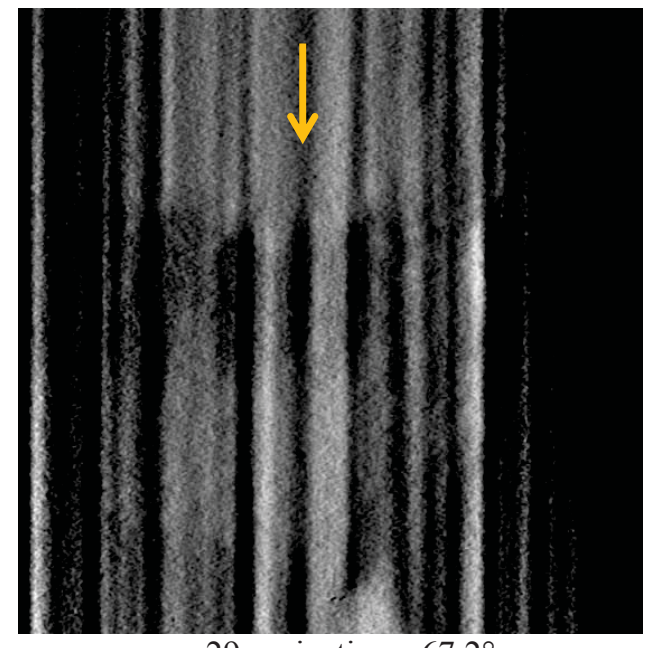

29 projections, $67.2^{\circ}$

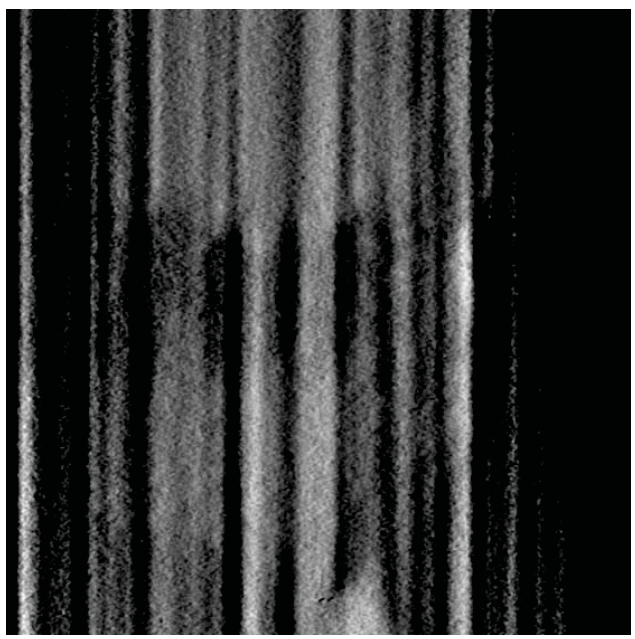

21 projections, $48^{\circ}$

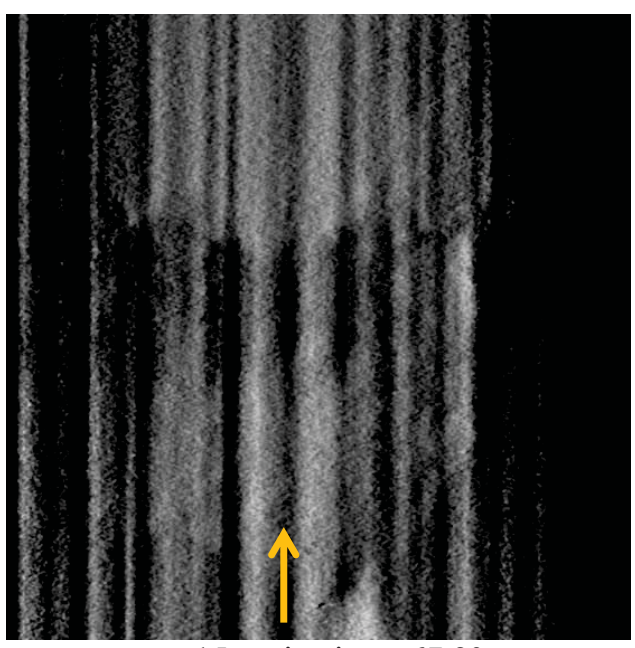

15 projections, $67.2^{\circ}$

Figure 6.7. Longitudinal reconstructions at azimuth $=30^{\circ}$ and $\mathrm{z}=0 \mathrm{~mm}$.

Arrows denote position of columns corresponding to fuel. 


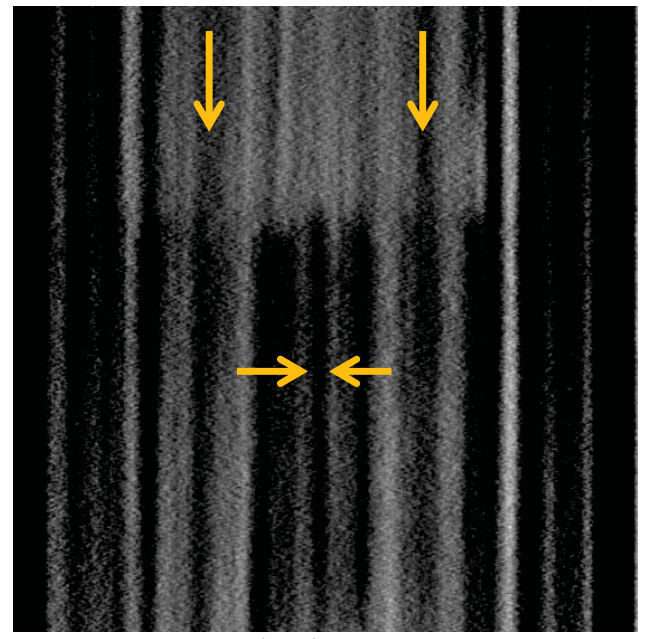

76 projections, $180^{\circ}$

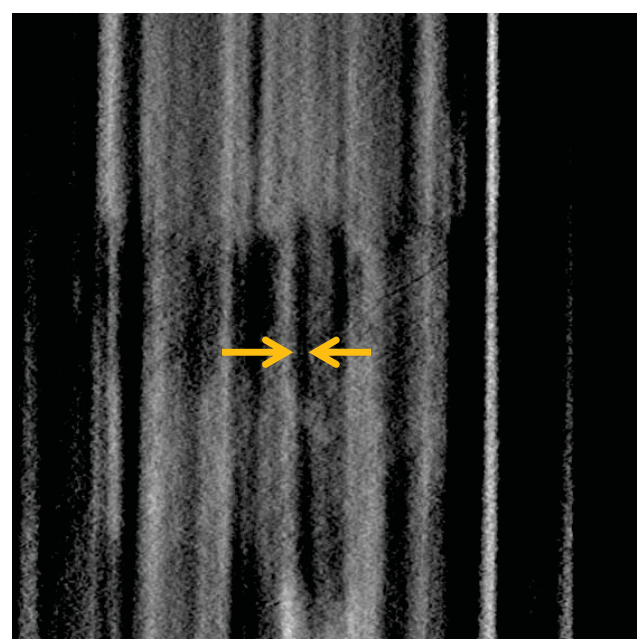

25 projections, $57.6^{\circ}$

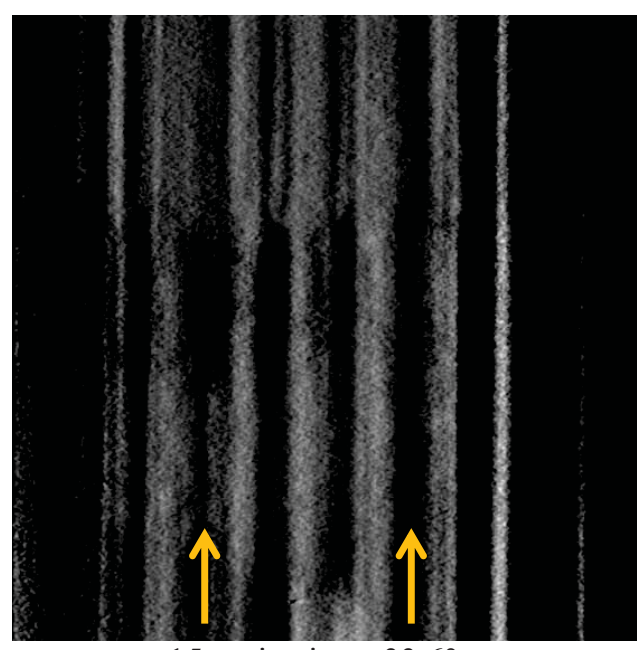

15 projections, $33.6^{\circ}$
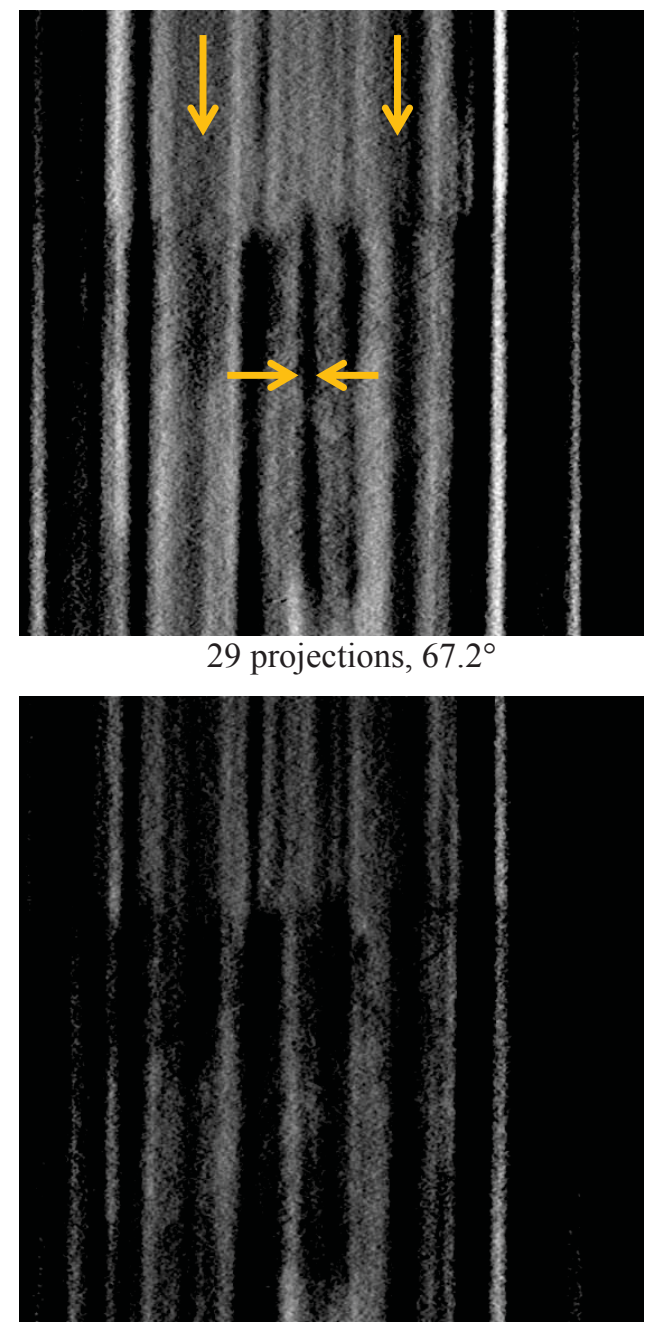

21 projections, $48^{\circ}$

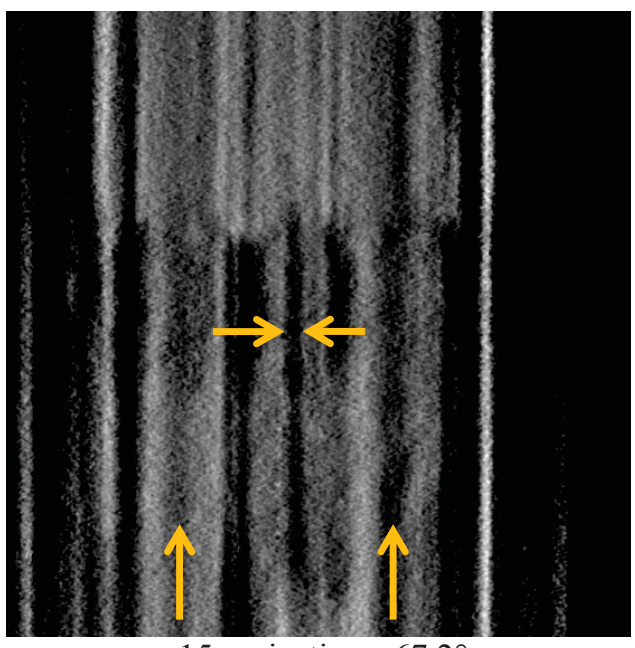

15 projections, $67.2^{\circ}$

Figure 6.8. Longitudinal reconstructions at azimuth $=30^{\circ}$ and $\mathrm{z}=3.5 \mathrm{~mm}$. 
Vertical arrows denote position of columns corresponding to fuel and horizontal arrows denote boundaries of partially in-plane structure.

From the longitudinal views at the zero azimuth angle (Figure 6.5 and Figure 6.6), the fuel pins are clearly visualized in all the combinations of projection numbers and angular range. Finer detail structures appear to be more sharply defined at the narrower angular ranges and appear softer at the wider angular ranges. These results are consistent with results from studies on tomosynthesis microcalcification visualization, where microcalcifications were slightly better visualized at narrower angular ranges as opposed to wider angular ranges. ${ }^{22}$ This general trend relates to the obliquity of the source images. However, the apparent sharpness with smaller angular ranges may also be the result of projection image mis-registration; reconstructions using large angular ranges could amplify the effect of projections misregistrations resulting in more blurring in the final reconstruction.

The longitudinal reconstructions at the $30^{\circ}$ azimuth view angle show the fuel pins from an oblique view angle. Reconstructing at this oblique angle simulates the angled viewpoints that will be necessary to optimally view the curved plate structure of the AFIP-7 assembly. Although not an optimal target for testing oblique reconstruction, the hexagonal column arrangement of the TREAT assembly did allow for some analysis of the oblique reconstruction image behavior.

At the oblique $\mathrm{z}=0 \mathrm{~mm}$ views (Figure 6.7), the effects of altering the acquisition angular range can be seen. With the full 76 projection, $180^{\circ}$ acquisition angle, the central fuel pin is visible and the out-ofplane pin structures on either side are largely blurred out. As the acquisition angle decreases, the z-axis (inter-plane) spatial resolution decreases. As a consequence, the out-of-plane structures become increasingly visible as the acquisition angle decreases.

Moving the reconstruction plane to $\mathrm{z}=3.5 \mathrm{~mm}$ at the same azimuth view angle of $30^{\circ}$ (Figure 6.8), two fuel pins are visible on the left and right with a small columnar structure in the center of the image (perhaps corresponding to the reconstruction plane partially intersecting with an out-of-plane fuel pin). With the complete 76 projection, $180^{\circ}$ data, this central structure is visible as a gap between two edges. As the acquisition angle is decreased, detail around this central gap gets softer. The gap in the center of the image remains visible up to the 25 -projection, $57.6^{\circ}$ reconstruction. Since we are viewing a reconstruction $30^{\circ}$ off of the central projection, we would expect that optimal lateral resolution at the $30^{\circ}$ azimuth view angle would be achieved with an acquisition angle large enough to include the $30^{\circ}$ projection direction. In the reconstructed images, we can see a detail structure in this central gap disappear when the projection angular range shrinks too far past the desired oblique view angle. 


\subsection{OSU Digitally Captured Images}

\subsubsection{Methods and Materials}

Digital projection images of an AFIP-7 fuel assembly were acquired by OSU using a prototype multichannel plate (MCP) digital detector, as shown in Figure 6.9. Projection images were acquired over $180^{\circ}$ in $0.25^{\circ}$ increments.

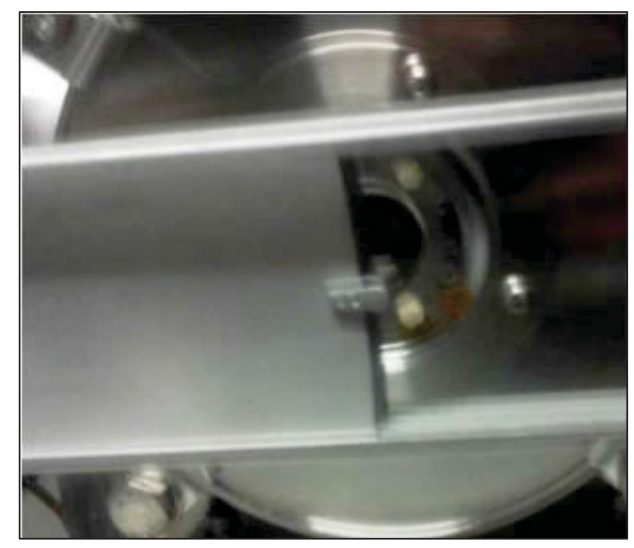

a) Picture of the assembly and MCP detector.

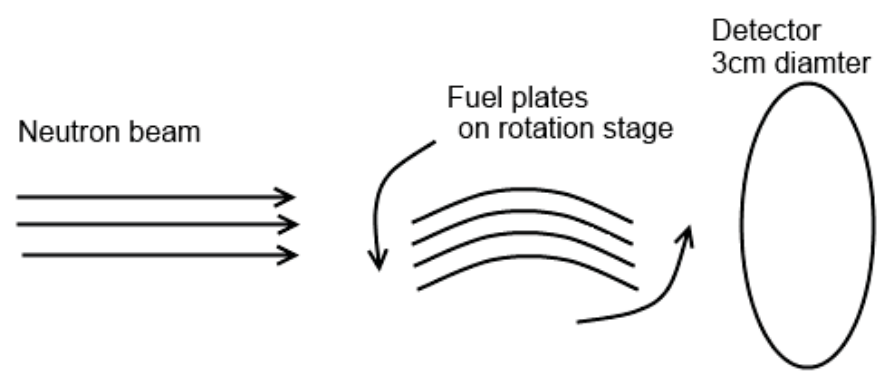

Figure 6.9. MCP detector and imaged plate assembly (a) and a diagram of image acquisition setup (b).

The projection images were first grayscale normalized. The non-valid background pixels were then segmented from the projection image pixels. Some of the pixels around the edge of the projection image did not correspond to real data from the detector. The segmented background region was dilated by two pixels to remove some of these invalid pixels from the projection image. The projections were then processed using contrast enhancing and sharpening filters. Finally, non-linear noise reduction filter was applied to suppress the noise in the projections.

Subsets of this data were used to perform tomosynthesis reconstructions at several angular ranges with different angle spacings between projections. The various combinations did not appear to substantially affect the image quality, so only results from the $14^{\circ}$ and $30^{\circ}$ angular ranges with $1^{\circ}$ projection spacings are presented as representative tomosynthesis reconstructions. Two different reconstructions were performed using these angular ranges: one oriented to view the plate holder end-on and another to view the fuel plates end-on.

\subsubsection{Results}

Representative reconstructions using the OSU projections are shown in Figure 6.10. While structure can be seen in the reconstructions, the images are noisy and lack clarity. There are a number of factors affecting the reconstruction image quality. First, there was some uncertainty in the acquisition geometry itself. The rotation axis was not known beforehand and was visually estimated from the projection images. Incorrect positioning of the axis of rotation leads mis-registration of the projections and blurring in the reconstruction.

Several characteristics of the projection images also affect to the reconstruction image quality. There were several inter-projection artifacts, including image position shifts, grayscale changes, and changes in detector field of view. Table 6.3 summarizes these artifacts. 

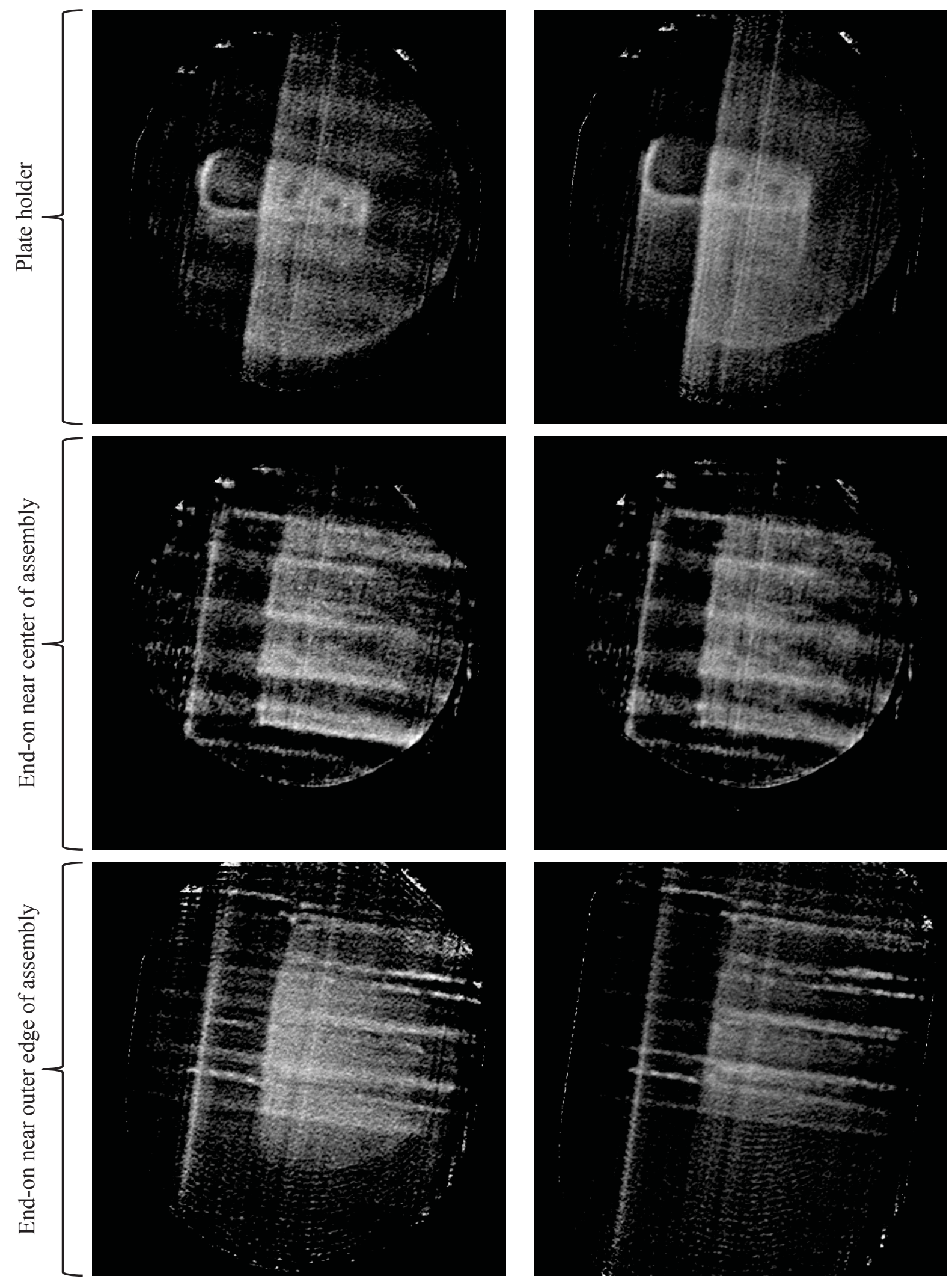

Left column: 15 projection, $14^{\circ}$ reconstructions.

Right column: 31 projection, $30^{\circ}$ reconstructions.

Figure 6.10. Reconstructions using the OSU projections. 
Table 6.3. Projection artifacts noted from OSU digital image acquisition.

\begin{tabular}{|l|l|}
\hline Projections & Observed Artifacts \\
\hline $101-102$ & Position shift \\
$181-182$ & Circular field of view region size changes \\
$476-232$ & Position shift \\
$499-502$ & Contrast change/grayscale shift \\
$601-602$ & Drastic grayscale change, position shift \\
$616-619$ & Grayscale shift \\
$662-664$ & Drastic grayscale change, position jitter \\
$715-718$ & Drastic grayscale change, position jitter \\
\hline
\end{tabular}

From discussions with OSU, technical issues with the MCP detector can cause the calibration of the detector to drift over time. The grayscale and contrast shifts, while not ideal, can be partially corrected via normalization. The inter-projection position jitter is more problematic in that it is another source of registration error which leads to unsharpness in the reconstructed image.

The initial set of projection images also exhibited low dynamic range and high noise. The projection images spanned between 40 and 50 grayscale values - slightly less than 6-bits of dynamic range (See Figure 11).
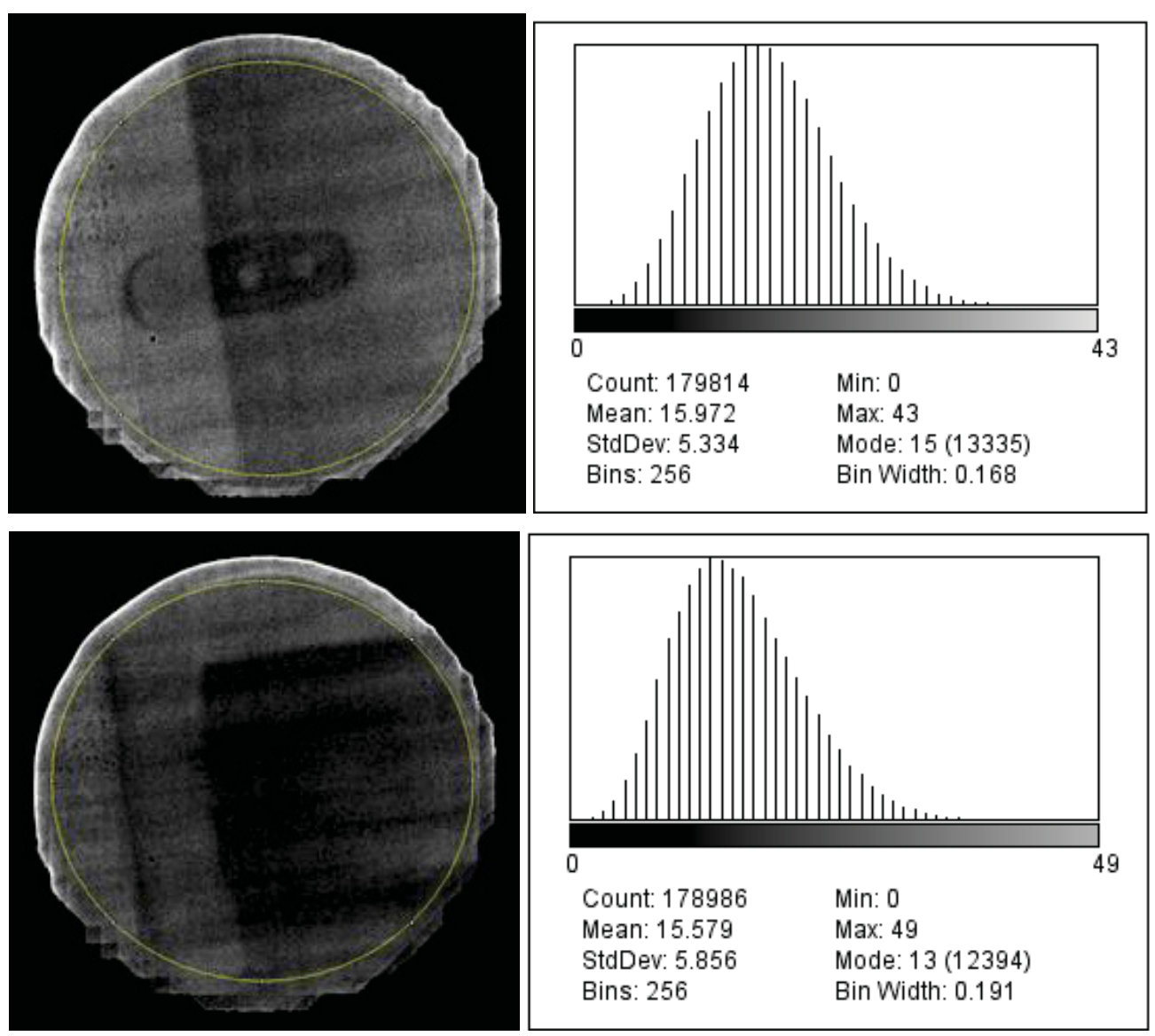

Figure 6.11. Regions of interest and corresponding histograms from two orthogonal projection images. 
Small variations in radiation absorption by the imaged target may not be adequately resolved with a small detector dynamic range. Since the fuel plates were not high contrast targets, detail can be lost from the subtle radiation absorption variations not being adequately captured. While there is sufficient contrast to visualize some targets, high image noise masks edges and other fine details, as depicted in Figure 6.12. If we average through a small region of interest, a profile plot reveals a clear delineation in gray values between different structural regions. However, if we take a line plot, we can see that the noise has a similar magnitude to the pixel value differences between regions. The noise level is high enough to confound the delineation of structural boundaries. Some of this noise can be mitigated though noise reduction filtering and the inherent noise-reducing integration from reconstructing with multiple projections; still, some loss of detail should be expected given the combination of low dynamic range, low contrast targets, and high noise in the digital projection images.
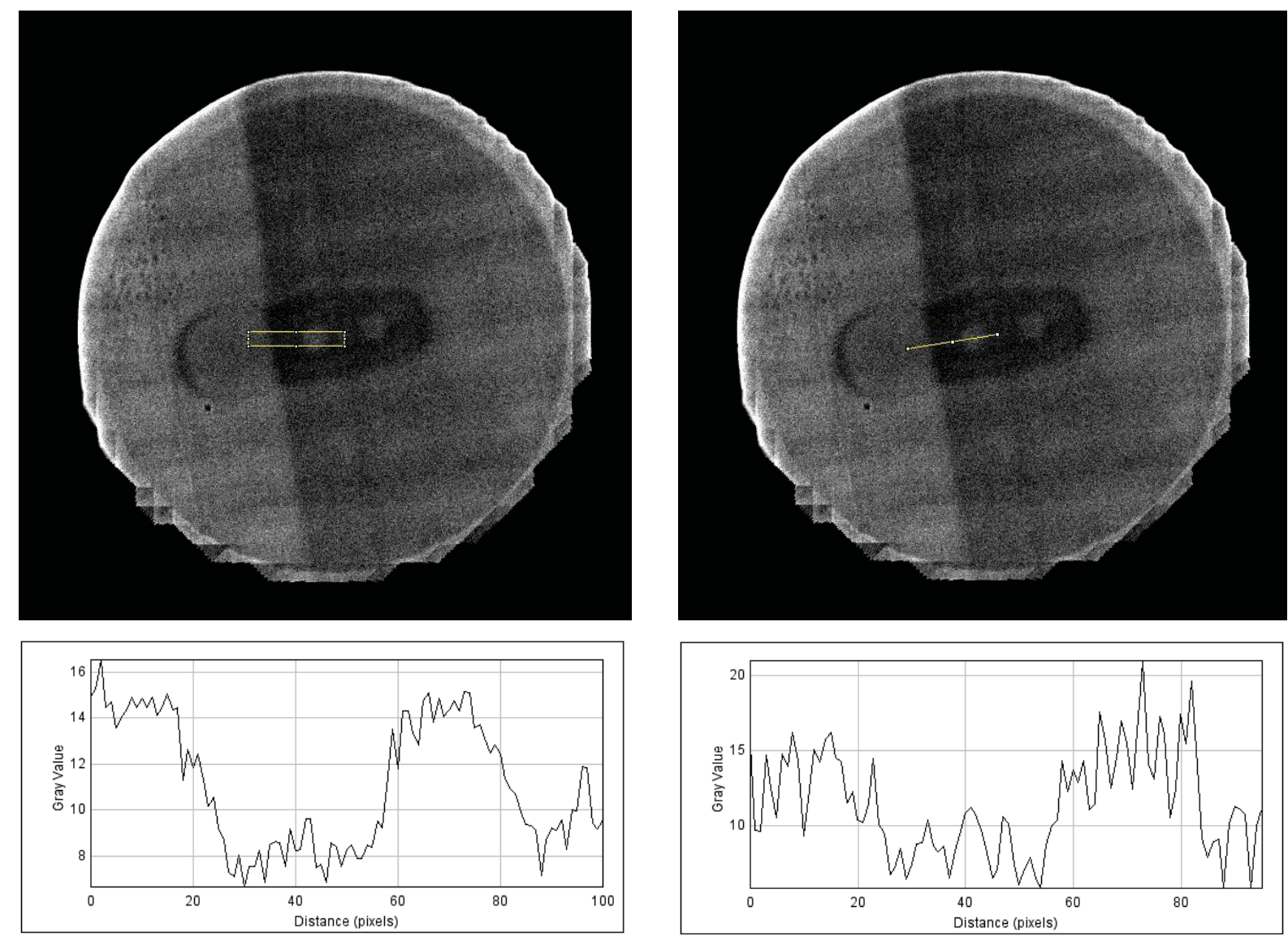

a) Plot profile of a rectangular ROI.

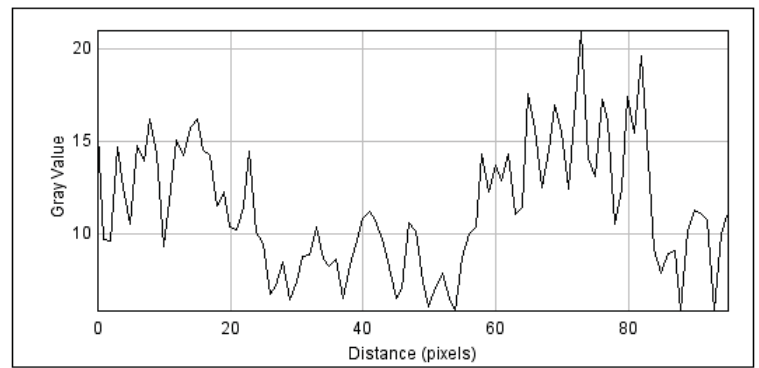

b) Plot profile of a linear ROI.

Figure 6.12. Projection image profile plots through the relatively high contrast plate holder. 


\subsection{Recommendations and Considerations}

The following are recommendations and guidelines for directing the acquisition to optimize the reconstruction based on the analyses summarized in this section:

- Use of a calibration phantom. A calibration phantom with known dimensions will facilitate the parameterization and optimization of the acquisition geometry and provide a more accurate reconstruction.

- Use fiducial markers for registration. Having properly registered images is important for optimal spatial resolution in the reconstruction. There are a number of steps in the film acquisition/digitization process where the image can shift. To correct for these positional errors, the projection images should be spatially co-registered prior reconstruction. This registration process could be facilitated by placing small stationary fiducial targets (or other unique identifiable features) in the projection image field-of-view. Assuming rigid image transformations, there should be at least two distinct fiducial targets in the image, ideally placed relatively far apart.

- Accurately determine the acquisition geometry. Reconstructing tomographic images requires accurate the knowledge of the image acquisition geometry. As a first pass, the mechanical setup (position of rotation axis, projection angles, detector position and orientation, etc.) of the acquisition system should be measured as accurately as possible. Ideally, the acquisition geometry should then be verified by imaging a calibration phantom with several point targets of known spatial distribution, as depicted in Figure 6.13.

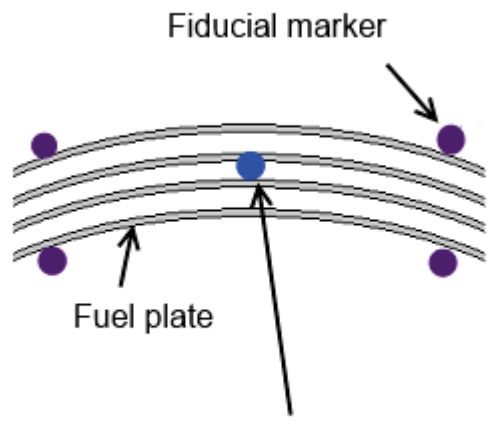

Center of rotation

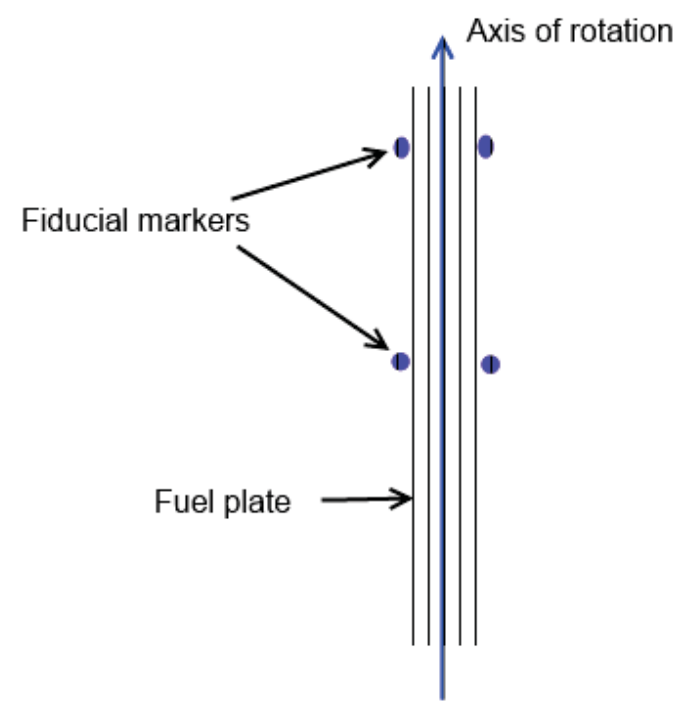

Figure 6.13. Top and side views of a suggested fiducial marker arrangement to validate the acquisition geometry.

- Acquisition angle should at least match the curvature of AFIP-7 plates. Lateral (in-plane) reconstruction resolution will be best when the projection angles include views at or near the desired reconstruction view angles. The ends of the fuel plates are angled at $\pm 17.7^{\circ}$ relative to the center of the plate (Figure 6.14). Given the constraint of minimizing the number of projections, the projection acquisition angle should also span at least $\pm 17.7^{\circ}$ to optimize the lateral resolution of the angled reconstruction planes used to visualize the gaps at the ends of the fuel plates. 


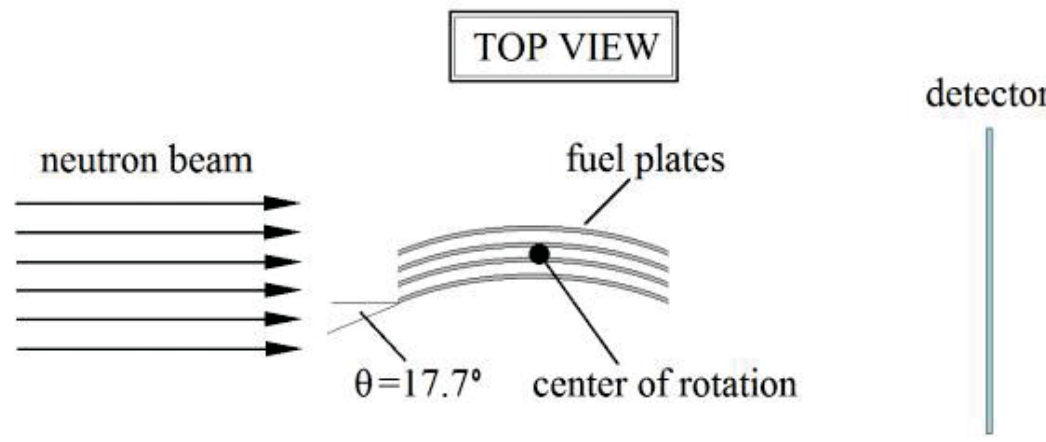

Figure 6.14. Illustration of image acquisition geometry for the AFIP-7 fuel element.

- More projections are desirable, if time and cost makes the acquisition feasible. If possible, enough projections should be used within the initial acquisition angle of $35.4^{\circ}\left( \pm 17.7^{\circ}\right)$ to bring the projection angular sampling closer to $1^{\circ}$. This should allow for super-resolution (reconstruction spatial resolution which is finer than the detector resolution) for properly registered projection images. If this angular sampling is achieved, additional projections should be used to extend the acquisition angular range. Tomosynthesis has good in-plane lateral (x-y plane) resolution, but poor inter-plane (z-direction) resolution due to the incomplete spatial sampling from the limited angle acquisition. Because the imaging of the fuel plates will be done "end-on", there will be significant structure oriented in z-direction which could clutter the reconstructed tomographic image slice with shadows from out-of-plane structure. Larger acquisition angles will spatially sample the target with more information in the z-direction and allow finer axial resolution to reduce the confounding out-of-plane shadowing.

- A digital acquisition is desirable long-term as it will simplify the image acquisition process, saving time and reducing the number of steps where errors can be introduced. At present, the digital detector may be a bit early in its development for routine use. Technical improvements will be necessary to improve the digital detector image quality. In addition, the small $(3 \mathrm{~cm})$ field of view of the digital detector would require stitching together multiple acquisitions to image the full length of a fuel assembly. For the time being, digitized radiograph acquisitions are more practical. 


\section{SUMMARY AND RESULTS}

This work seeks to assess the geometric stability of the U-Mo monolithic fuel system evaluating the radiation-induced changes in the AFIP-7 experiment device. Neutron radiography and tomography provide valuable information about the post-irradiation condition of the fuel specimen. Tomographic reconstructions of the AFIP-7 fuel element will be analyzed to assess the geometric condition of the element after irradiation and provide information regarding the condition of the fuel, including gross geometric defects, bowing, twist, plate buckling, cracks, and other defects. The INL, in collaboration with Oregon State University, Missouri University of Science and Technology, and Real Time Tomography, are developing advanced neutron detector systems and tomographic reconstruction techniques for this project. Neutron computed tomography using the current neutron radiography technique is impractical due to the long time and high cost needed to acquire the set of images for tomographic reconstruction. Advanced neutron radiography systems such as the MCP detector and neutron computed radiography may reduce the time and cost of acquiring images for neutron $\mathrm{CT}$.

The MCP detector system tested at OSU and Missouri S\&T provides nearly real-time neutron radiographs and has lower gamma sensitivity compared to other real-time acquisition image systems. However, the system is far less of a "turn-key" system than previously anticipated, and some significant, but not prohibitive, challenges must be overcome to make its use for imaging nuclear fuel more practical. Images taken with the MCP requires significant image processing to reduce distortions and correct for the dynamic detector response. The dynamic detector response requires acquisition of multiple flat-field images for flat-field correction of the radiographs; this would become prohibitively burdensome at the NRAD, as the fuel specimen must be moved out of the field of view for each flat-field image. If the dynamic detector response issues are resolved, the active area $(\sim 30 \mathrm{~mm}$ diameter) of the MCP requires the MCP to collect and digitally combine several images of a specimen, which may become time consuming, but not impossible. The MCP detector was to be tested in a high-radiation environment at the INL in summer 2013, but the detector was damaged in shipment. These tests should go forward once the $\mathrm{MCP}$ detector is repaired and operational.

In addition to the MCP detector system, the INL is developing neutron computed radiography (CR), which uses the same foil transfer method currently employed at the NRAD, but replaces the film with a photostimulable storage phosphor imaging plate that is sensitive to the decay radiation from the activated transfer foils. Preliminary tests show that the spatial resolution properties of neutron CR may be comparable to film radiography images using the existing neutron radiography technique at NRAD. However, there are many factors that affect the spatial resolution of the radiographs that may make images produced with neutron CR that have spatial resolution properties that exceed film radiography. High-resolution imaging plates combined with a high-resolution scanner will likely allow for such improvements in spatial resolution of neutron CR. Further development of neutron CR may produce images of comparable or better quality than currently available, and would reduce the cost and time required to acquire the images. The reduction in cost may be significant, which would make neutron

imaging more appealing to existing programs that use neutron radiography at INL and may attract additional demand for this rare and valuable capability.

Because transfer method neutron radiography is expensive, acquisition of a full set of radiographs for tomographic reconstruction is impractical. The INL is developing tomographic reconstruction techniques that require only a small set of radiographs of the AFIP-7 fuel element. These tomosynthesis techniques are tested using archived radiographs of TREAT experiments for CT as a proof of principle. These same techniques are also applied to radiographs taken with an MCP detector of a mock-up of the AFIP-7 geometry.

Acquisition angle should at least match the curvature of AFIP-7 plates. Lateral (in-plane) reconstruction resolution will be best when the projection angles include views at or near the desired reconstruction view angles. The ends of the fuel plates are angled at $\pm 17.7^{\circ}$ relative to the center of the plate. Increasing the 
number of projections is desirable, but given the constraint of minimizing the number of projections, the projection acquisition angle should also span at least $\pm 17.7^{\circ}$ to optimize the lateral resolution of the angled reconstruction planes used to visualize the gaps at the ends of the fuel plates. If possible, enough projections should be used within the initial acquisition angle of $35.4^{\circ}\left( \pm 17.7^{\circ}\right)$ to bring the projection angular sampling closer to $1^{\circ}$.

Further testing will determine a more specific recommendation for the number and angular distribution of neutron radiographs that will produce the highest quality tomographic reconstruction of the AFIP-7 fuel element. 


\section{REFERENCES}

1. Domanus, J.C., Practical Neutron Radiography, Kluver Academic Publishers, Dordrecht (1992)

2. Berger, H. (editor), Practical Applications of Neutron Radiography and Gaging, American Society for Testing and Materials, STP 586, Gaithesburg, MD, February 10-11 (1976)

3. Richards, W.J. and McClellan, G.C., "Neutron Radiography at the Hot Fuel Examination Facility," Proceedings of the 27th Conference on Remote Systems Technology, pp. 203-208 (1979)

4. Bossi, R.H., Iddings F.A., and Wheeler, G.C., Nondestructive Testing Handbook, Vol. 4. Radiographic Testing, American Society for Nondestructive Testing, Inc., USA (2002)

5. McClellan, G.C. and Richards, W.J., "Neutron Radiography at the NRAD Facility," Proceedings of the Ninth U.S. TRIGA User's Conference, CONF-840386-3, Anaheim, CA, March 12-14 (1984)

6. Berger, H., "Detection Systems for Neutron Radiography," Practical Applications of Neutron Radiography and Gaging, American Society for Testing and Materials, STP 586, pp. 35-57 (1976)

7. Lehmann, E.H., Frei, G., Kuhne, G., Boillat, P., "The Micro-Setup for Neutron Imaging: A Major Step Forward to Improve the Spatial Resolution," Nuclear Instruments and Methods, Sec. A, no. 576, pp. 389-396 (2007)

8. A.S. Tremsin, W.B. Feller, and R.G. Downing, "Efficiency Optimization of Microchannel Plate (MCP) Neutron Imaging Detectors," Nuclear Instruments and Methods in Physics Research, Section A: Accelerators, Spectrometers, Detectors and Associated Equipment, vol. 539, 1-2 (2005)

9. Oswald, H. Siegmund, J.V. Vallerga, A.S. Tremsin, J. Mcphate, and B. Feller, "High spatial resolution neutron sensing microchannel plate detectors," Nuclear Instruments and Methods in Physics Research, Section A: Accelerators, Spectrometers, Detectors and Associated Equipment, vol. 576, 1 (2007)

10. ASTM International E5454-05, Standard Test Method for Determining Image Quality in Direct Thermal Neutron Radiographic Examination, American Society for Testing and Materials, ASTM E545-05 (2005)

11. American Society for Testing and Materials International, "Standard practice for Fabrication of the Neutron Radiographic Sensitivity Indicator," American Society for Testing and Materials, ASTM E2023-10 (2010)

12. American Society for Testing and Materials International, "Standard practice for Fabrication of the Neutron Radiographic Beam Purity Indicators," American Society for Testing and Materials, ASTM E2003-10 (2010)

13. inCT, Octopus Manual, Ghent, Belgium, vol. 8.5 (2008)

14. Hardt, P.V.D., Neutron Radiography Handbook, D. Reidel Pub. Co. (1981)

15. Goshtasby A., "Piecewise linear mapping functions for image registration," Pattern Recognition, vol. 19, no. 6, pp. 459-466 (1986)

16. Fonseca, L.M.G. and Manjunath, B.S., "Registration techniques for multisensory remotely sensed imagery," American Society for Photogrammetric Engineering \& Remote Sensing, vol. 62, pp. 10491056. (1996)

17. Abir, M.I.K., Islam, F.F., Wachs, D. M. and Lee, H.K., "Multiscale based adaptive contrast enhancement," SPIE Electronic Imaging, SPIE vol. 8657, pp. 86570X-1-9 (2013)

18. Zeng G. L., Medical Image Reconstruction, Beijing and Springer-Verlag: Berlin Heidelburg, (2010) 
19. Morman, J. and Froehle, P., "Tomographic reconstruction of fuel assembly configurations:

PFR/TREAT test L07 lower blockage region," ANL/RAS, Argonne, IL (1989)

20. Acciavatti, R. and Maidment, A., "Observation of super-resolution in digital breast tomosynthesis," Med. Phys., pp. 7518-7539 (2012)

21. Kuo, J., Ringer, P., Fallows, S., Bakic, P., Maidment, A., and Ng, S., "Dynamic reconstruction and rendering of 3D tomosynthesis images," in Proc. SPIE 7961 Medical Imaging, Orlando, FL (2011)

22. Lu, Y., Chan, H., Wei, J., Goodsitt, M., Carson, P., Hadjiisk, L., Schmitz, A., Ebergard, J. and B. Claus, "Image quality of microcalcifications in digital breast tomosynthesis: effects of projectionview distributions," Med. Phys., pp. 5703-5712 (2011) 